\title{
Gemini-GRACES high-quality spectra of Kepler evolved stars with transiting planets
}

\section{Detailed characterization of multi-planet systems Kepler-278 and Kepler-391}

\author{
E. Jofré ${ }^{1,2,10}$, J. M. Almenara ${ }^{3}$, R. Petrucci ${ }^{1,2,10}$, R. F. Díaz ${ }^{4,5}$, Y. Gómez Maqueo Chew ${ }^{2}$, E. Martioli ${ }^{6}$, I. Ramírez ${ }^{7}$, \\ L. García ${ }^{1}$, C. Saffe ${ }^{8,9,10}$, E. F. Canul ${ }^{2}$, A. Buccino ${ }^{4,5}$, M. Gómez ${ }^{1,10}$, and E. Moreno Hilario ${ }^{2}$ \\ ${ }^{1}$ Observatorio Astronómico de Córdoba, Universidad Nacional de Córdoba, Laprida 854, X5000BGR Córdoba, Argentina \\ e-mail: emiliano@astro.unam.mx \\ ${ }^{2}$ Instituto de Astronomía, Universidad Nacional Autónoma de México, Ciudad Universitaria, CDMX, C.P. 04510, Mexico \\ ${ }^{3}$ Observatoire de Genève, Département d'Astronomie, Université de Genève, Chemin des Maillettes 51, 1290 Versoix, Switzerland \\ ${ }^{4}$ Universidad de Buenos Aires, Facultad de Ciencias Exactas y Naturales, Buenos Aires, Argentina \\ ${ }^{5}$ CONICET, Universidad de Buenos Aires, Instituto de Astronomía y Física del Espacio (IAFE), Buenos Aires, Argentina \\ ${ }^{6}$ Laboratorio Nacional de Astrofísica, rua Estados Unidos 154, Itajubá, MG, Brazil \\ 7 Tacoma Community College, 6501 South 19th Street, Tacoma, WA 98466, USA \\ ${ }^{8}$ Instituto de Ciencias Astronómicas, de la Tierra y del Espacio, C.C 467, 5400 San Juan, Argentina \\ ${ }^{9}$ Facultad de Ciencias Exactas, Universidad Nacional de San Juan, Físicas y Naturales, San Juan, Argentina \\ ${ }^{10}$ Consejo Nacional de Investigaciones Científicas y Técnicas (CONICET), Godoy Cruz 2290, CABA, CPC 1425FQB, Argentina ]
}

Received 2 August 2019 / Accepted 17 December 2019

\begin{abstract}
Aims. Kepler-278 and Kepler-391 are two of the three evolved stars known to date on the red giant branch (RGB) to host multiple short-period transiting planets. Moreover, the planets orbiting Kepler-278 and Kepler-391 are among the smallest discovered around RGB stars. Here we present a detailed stellar and planetary characterization of these remarkable systems.

Methods. Based on high-quality spectra from Gemini-GRACES for Kepler-278 and Kepler-391, we obtained refined stellar parameters and precise chemical abundances for 25 elements. Nine of these elements and the carbon isotopic ratios, ${ }^{12} \mathrm{C} /{ }^{13} \mathrm{C}$, had not previously been measured. Also, combining our new stellar parameters with a photodynamical analysis of the Kepler light curves, we determined accurate planetary properties of both systems.

Results. Our revised stellar parameters agree reasonably well with most of the previous results, although we find that Kepler- 278 is $\sim 15 \%$ less massive than previously reported. The abundances of $\mathrm{C}, \mathrm{N}, \mathrm{O}, \mathrm{Na}, \mathrm{Mg}, \mathrm{Al}, \mathrm{Si}, \mathrm{S}, \mathrm{Ca}, \mathrm{Sc}, \mathrm{Ti}, \mathrm{V}, \mathrm{Cr}, \mathrm{Mn}, \mathrm{Co}, \mathrm{Ni}, \mathrm{Cu}$, $\mathrm{Zn}, \mathrm{Sr}, \mathrm{Y}, \mathrm{Zr}, \mathrm{Ba}$, and $\mathrm{Ce}$, in both stars, are consistent with those of nearby evolved thin disk stars. Kepler-391 presents a relatively high abundance of lithium $\left(A(\mathrm{Li})_{\mathrm{NLTE}}=1.29 \pm 0.09 \mathrm{dex}\right)$, which is likely a remnant from the main-sequence phase. The precise spectroscopic parameters of Kepler-278 and Kepler-391, along with their high ${ }^{12} \mathrm{C} /{ }^{13} \mathrm{C}$ ratios, show that both stars are just starting their ascent on the RGB. The planets Kepler-278b, Kepler-278c, and Kepler-391c are warm sub-Neptunes, whilst Kepler-391b is a hot sub-Neptune that falls in the hot super-Earth desert and, therefore, it might be undergoing photoevaporation of its outer envelope. The high-precision obtained in the transit times allowed us not only to confirm Kepler-278c's TTV signal, but also to find evidence of a previously undetected TTV signal for the inner planet Kepler-278b. From the presence of gravitational interaction between these bodies we constrain, for the first time, the mass of Kepler- $278 \mathrm{~b}\left(M_{\mathrm{p}}=56_{-13}^{+37} M_{\oplus}\right)$ and Kepler-278c $\left(M_{\mathrm{p}}=35_{-21}^{+9.9} M_{\oplus}\right)$. The mass limits, coupled with our precise determinations of the planetary radii, suggest that their bulk compositions are consistent with a significant amount of water content and the presence of $\mathrm{H}_{2}$ gaseous envelopes. Finally, our photodynamical analysis also shows that the orbits of both planets around Kepler-278 are highly eccentric $(e \sim 0.7)$ and, surprisingly, coplanar. Further observations (e.g., precise radial velocities) of this system are needed to confirm the eccentricity values presented here.
\end{abstract}

Key words. stars: fundamental parameters - stars: abundances - stars: individual: Kepler-278 - planetary systems techniques: spectroscopic - stars: individual: Kepler-391

\footnotetext{
* The reduced spectra (FITS files) are only available at the CDS via anonymous ftp to cdsarc.u-strasbg.fr (130.79.128.5) or via http:// cdsarc.u-strasbg.fr/viz-bin/cat/J/A+A/634/A29

${ }^{\star \star}$ Based on observations obtained at the Gemini Observatory, which is operated by the Association of Universities for Research in Astronomy, Inc., under a cooperative agreement with the NSF on behalf of the Gemini partnership: the National Science Foundation (United States), National Research Council (Canada), CONICYT (Chile), Ministerio de Ciencia, Tecnología e Innovación Productiva (Argentina), Ministério da Ciência, Tecnologia e Inovação (Brazil), and Korea Astronomy and Space Science Institute (Republic of Korea).
} 


\section{Introduction}

To date, radial velocity (RV) surveys for planets around stars that evolved off the main-sequence, such as subgiants and giants, have resulted in the discovery of more than 150 planets (e.g., Johnson et al. 2007; Niedzielski et al. 2009; Döllinger et al. 2009; Sato et al. 2010). These detections have been crucial for extending the studies of planet-star connections to stars more massive than the Sun. The analysis of precise spectroscopic metallicities of the subgiant hosts has revealed that these stars follow the same gas-giant planet-metallicity correlation found for dwarf stars (e.g., Fischer \& Valenti 2005; Ghezzi et al. 2010a; Jofré et al. 2010, 2015b; Maldonado et al. 2013), but the presence of planets around giant stars does not seem to be sensitive to the metallicity of their hosts (e.g., Ghezzi et al. 2010a; Maldonado et al. 2013; Mortier et al. 2013; Jofré et al. 2015b); see, however, the discussion in Reffert et al. (2015). Moreover, precise determinations of stellar masses in evolved stars together with the results from the surveys around FGKM dwarfs show that giant planet occurrence also increases with stellar mass (e.g., Lovis \& Mayor 2007; Johnson et al. 2010; Ghezzi et al. 2018).

Regarding the properties of planets around evolved stars, one of the most important trends revealed by the RV searches is a paucity of close-in planets. In particular, there is a lack of planets orbiting closer than $\sim 0.5 \mathrm{AU}(P \lesssim 100$ days) to giant or subgiant stars (e.g., Johnson et al. 2007; Niedzielski et al. 2009; Sato et al. 2008, 2010). Several scenarios have been proposed to explain the observed distribution. The first idea suggests that planets are destroyed as they spiral into their host stars as a result of tidal interactions (Villaver \& Livio 2009; Kunitomo et al. 2011). In a second scenario, planet formation and evolution mechanisms around stars more massive than the Sun, including the shorter lifetime of the inner protoplanetary disks, promote the lower frequency of gas giant planets at short orbital distances (e.g., Johnson et al. 2007; Burkert \& Ida 2007; Currie 2009; Kretke et al. 2009). Another possibility is that short period RV stellar oscillations may mask the detection of short period planets that still might reside very close to their stars (Pasquini et al. 2008).

Detections of planetary transits around evolved stars are extremely challenging because their large radii cause not only shallow transit depths ${ }^{1}$ but also long transit durations. Recently, high-precision photometry obtained with the Kepler and K2 missions have allowed the discovery of a handful $(\sim 20)$ transiting planets around stars with $\log g<3.7 \mathrm{dex}^{2}$. Interestingly, in striking contrast to the radial velocity results, most of these are close-in planets with semi-major axis between $\sim 0.06 \mathrm{AU}$ and 0.3 AU. The detection and detailed characterization of these planetary systems are crucial for constraining theories of planet-star interaction (Lillo-Box et al. 2014; Quinn et al. 2015; Van Eylen et al. 2016; Chontos et al. 2019), models of planet inflation (e.g., Lopez \& Fortney 2016; Van Eylen et al. 2016; Grunblatt et al. 2016, 2018), and scenarios of planet formation in intermediate and high-mass stars (e.g., Burkert \& Ida 2007).

Accurate atmospheric stellar parameters $\left(T_{\text {eff }}, \log g\right.$, and $[\mathrm{Fe} / \mathrm{H}])$, derived from both high resolution and high signal-tonoise ratio (S/N) spectra (e.g., Sousa et al. 2011; Bedell et al. 2014), can be combined with stellar models (e.g., Demarque et al. 2004) to derive precise stellar masses, radii, and ages. However, given that most of the Kepler planet-candidate hosts

\footnotetext{
1 Transit depth scales inversely with the square of the stellar radius.

2 Around 15 planets are orbiting RGB stars.
}

(KOIs) are too faint $(V \gtrsim 12)$ to obtain high-quality spectra for all of them, their atmospheric parameters were derived first based on broadband photometric calibrations (Brown et al. 2011; Pinsonneault et al. 2012). It has been shown that these initial parameters present limited accuracies (Molenda-Żakowicz et al. 2011; Bruntt et al. 2011, 2012; Thygesen et al. 2012; Huber et al. 2014) causing uncertainties of $\approx 42 \%$ in the determination of the stellar mass and $\approx 16 \%$ in radius (Johnson et al. 2017), and ultimately affecting the derived planetary properties significantly.

Johnson et al. (2017) showed that a significant improvement in the precision of the stellar radius $(\sim 11 \%)$ and stellar mass $(\sim 4 \%)$ can be obtained when using high-resolution HIRES spectra to derive the fundamental parameters of a large sample of planet-candidate Kepler hosts (Petigura et al. 2017). Most of these stars, however, have spectra with $S / N \sim 40-70$ (Martinez et al. 2019), which may not be suitable for a detailed and precise chemical analysis that could reveal not only observable signatures of planet accretion (e.g., Adamów et al. 2012, 2014, 2015; Carlberg et al. 2012; Aguilera-Gómez et al. 2016; Meléndez et al. 2017) but also to provide better constraints on their evolutionary status (e.g., Gilroy \& Brown 1991; Carlberg et al. 2012).

In this context, we present a detailed stellar and planetary characterization of the exceptional planetary systems Kepler-278 and Kepler-391. These are two of the three short-period multitransiting planet systems, known to date, that transit evolved stars in the red giant branch $(\mathrm{RGB})^{3}$. Furthermore, the planet sizes are among the smallest discovered around RGB stars. Both Kepler-278 (KOI-1221, KIC 3640905; $V=11.8$ ) and Kepler-391 (KOI-2541, KIC 12306058; $V=13.2$ ) were observed by Kepler from 2009 until the end of its primary mission in 2013. Kepler278 was identified by Borucki et al. (2011) as hosting multiple Neptune-size planet candidates with periods of 30.1 and 51.1 days. Batalha et al. (2013) revealed a single periodic transit signal with a period of 7.4 days and depth consistent with a sub-Neptune size planet candidate around Kepler-391. Rowe et al. (2014), later reported an additional sub-Neptune size companion around Kepler-391 with a period of 20.5 days. All planets around both stars were statistically validated (Rowe et al. 2014; Lissauer et al. 2014; Morton et al. 2016). In addition, Van Eylen \& Albrecht (2015) first reported that Kepler-278c exhibits sinusoidal transit timing variations (TTV).

Our characterization includes the first determination of refined stellar parameters and precise photospheric chemical abundances of 25 elements based on high-quality GeminiGRACES spectra. We additionally performed a photodynamical modeling of the Kepler light curves that, in combination with our new stellar parameters, provides improved planetary properties. In particular, for the system Kepler-278 we were able to derive the eccentricity of both planets and, thanks to the presence of dynamical interactions in the system, we constrained, for the first time, the masses of the two planets.

In Sect. 2, we summarize the observations and data reduction. We present the determination of stellar parameters and the detailed chemical analysis in Sect. 3. We describe our photodynamical model and present our refined planetary parameters in Sect. 4. We discuss the resulting stellar and planetary properties of Kepler-278 and Kepler-391 in the context of other systems in Sect. 5. Finally, in Sect. 6, we summarize our findings and conclusions. 3 Kepler- 432 is a red giant star that also hosts two planets, but the outer
one does not transit the star. 


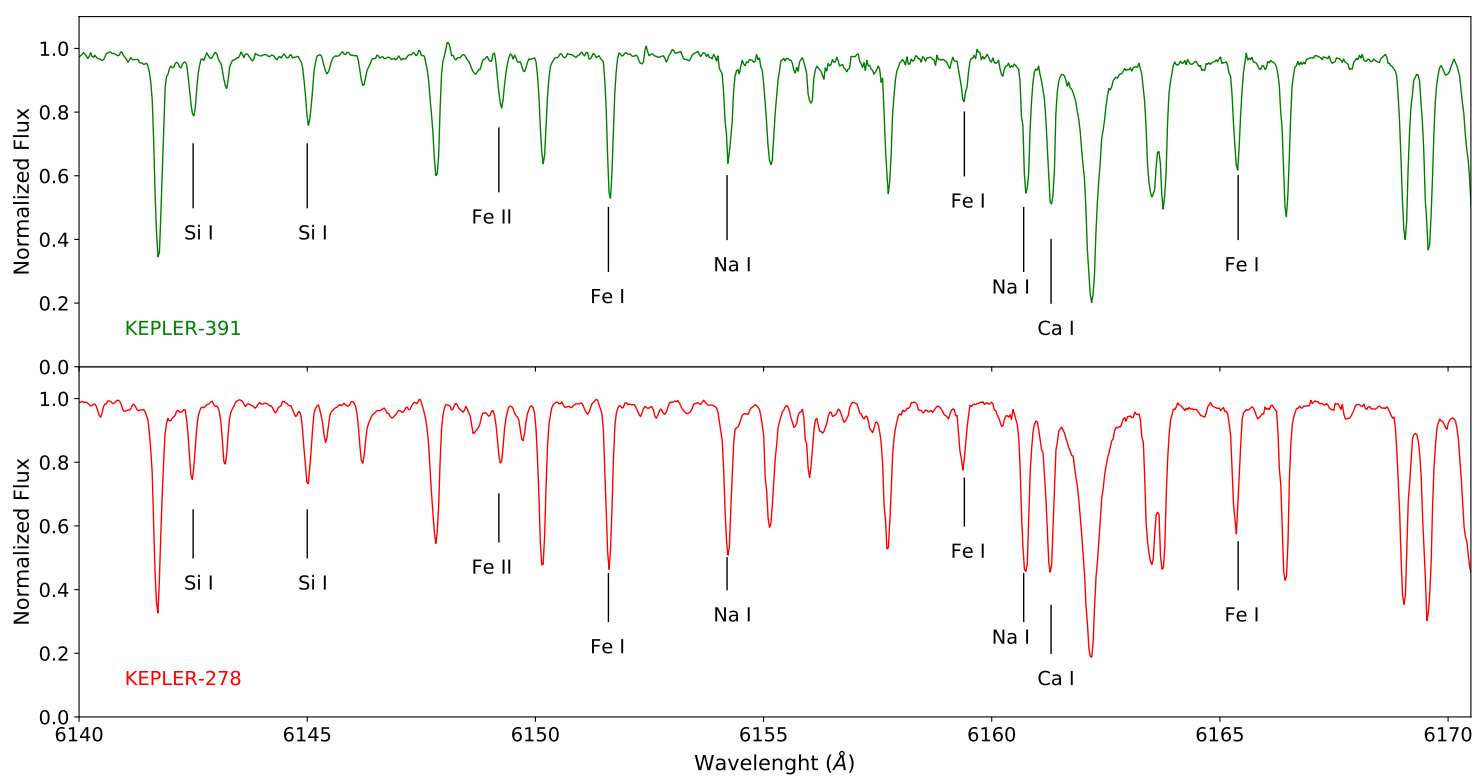

Fig. 1. Narrow range of the high-quality spectra of Kepler-391 (top) and Kepler-278 (bottom) obtained with GRACES at Gemini North Observatory. Some of the lines measured in this region to derive chemical abundances or $T_{\text {eff }}$ via the line depth ratios technique are labeled.

\section{Observations}

\subsection{GRACES spectra}

We observed Kepler-278 and Kepler-391 on 2016 September 10 UT with the Gemini Remote Access to CFHT ESPaDOnS Spectrograph (GRACES; Chene et al. 2014) at the 8.1-m Gemini North telescope. Observations were carried out in the queue mode (GN-2016B-Q-11, PI: E. Jofré) in the one-fiber mode (object only), which achieves a resolving power of $R \sim 67500$ between 400 and $1050 \mathrm{~nm}$. We obtained consecutive exposures of $2 \times 534 \mathrm{~s}$ for Kepler-278 and $2 \times 1124 \mathrm{~s}$ for Kepler-391.

These observations along with a series of calibrations including $6 \times$ ThAr arc lamp, $10 \times$ bias, and $7 \times$ flat-field exposures were used as input in the OPERA (Open source Pipeline for ESPaDOnS Reduction and Analysis) code ${ }^{4}$ software (Martioli et al. 2012) to obtain the reduced data. The reduction includes optimal extraction of the orders using a tilted oversampled aperture. The aperture tilt angle is calibrated on an oversampled instrument profile, which is measured from the ThAr spectrum. The reduction also comprises the wavelength calibration where the set of calibration lines are automatically detected by computing the cross-correlation between the ThAr spectrum and the measured instrument profile. The lines are identified using the Lovis \& Pepe (2007) ThAr atlas. The wavelength solution is obtained with an average accuracy of $\sim 60 \mathrm{~m} \mathrm{~s}^{-1}$. Also, OPERA performs the normalization of the spectra as follows. First, it divides the raw flux by the normalized blaze function obtained from flat-field exposures. Then it bins the spectrum by calculating the median of every $\sim 100$ points. For each bin, it calculates a local robust linear fit using two neighbor bins on each side, where the linear function is evaluated at the mean wavelength of the central bin providing an estimate to the continuum flux. Thus, the continuum is evaluated at each bin. To obtain the continuum value for each spectral element, it performs a cubic spline interpolation. Finally, it divides the flux by the continuum to obtain the normalized flux.

The two individual exposures of each target were co-added to obtain the final spectra with $\mathrm{S} / \mathrm{N}$ per resolution element of

4 OPERA is available at http://wiki.lna.br/wiki/espectro
$S / N \sim 450$ and $S / N \sim 300$ around $6700 \AA$, for Kepler-278 and Kepler-391, respectively. We derived absolute radial velocities (RVs) by cross-correlating our program stars with standard stars using the $\mathrm{IRAF}^{5}$ task fxcor, obtaining VR $=-46.98 \pm$ $0.04 \mathrm{~km} \mathrm{~s}^{-1}$ for Kepler-278 and VR $=21.59 \pm 0.05 \mathrm{~km} \mathrm{~s}^{-1}$ for Kepler-391. These values are in excellent agreement with the absolute RVs from Gaia Data Release 2 (DR2, Gaia Collaboration 2018) of $-46.73 \pm 0.83 \mathrm{~km} \mathrm{~s}^{-1}$ and $21.55 \pm 2.18 \mathrm{~km} \mathrm{~s}^{-1}$ for Kepler-278 and Kepler-391, respectively. Finally, the combined spectra were corrected for the radial velocity shifts with the IRAF task dopcor. A small portion of the spectra of both stars is shown in Fig. 1.

\subsection{Kepler light curve}

We retrieved the data release 25 of the Kepler light curves (Twicken et al. 2016) from the Mikulski Archive for Space Telescopes (MAST) ${ }^{6}$. Kepler-278 was observed from quarter Q1 to Q8 in long-cadence data (about one point every $29.4 \mathrm{~min}$ ), and from Q9 to Q17 in short-cadence data (about one point per minute). Kepler-391 was observed from quarter Q0 to Q17 only in long-cadence data. We used the simple aperture photometry (SAP) light curve, which we corrected for the flux contamination (between 0.0 and $1.0 \%$ depending on the quarter) using the value estimated by the Kepler team. Only the data spanning three transit durations around each transit were modelled, after normalization with a parabola. The observed transits for Kepler-278b/c and Kepler-391b/c are presented in Figs. B.2-B.5.

\section{Stellar parameters}

\subsection{Fundamental atmospheric parameters}

The atmospheric fundamental parameters $\left(T_{\mathrm{eff}}, \log g,[\mathrm{Fe} / \mathrm{H}]\right.$, and microturbulent velocity, $v_{\text {micro }}$ ) of Kepler-278 and Kepler-391 were derived by imposing spectroscopic equilibrium

5 IRAF is distributed by the National Optical Astronomy Observatories, which are operated by the Association of Universities for Research in Astronomy, Inc., under cooperative agreement with the National Science Foundation.

6 http://archive.stsci.edu 
of neutral and singly-ionized iron lines (Fe I and Fe II). Using the average of literature values for each star as the initial set of atmospheric parameters, all the parameters are iteratively modified until the correlations of $[\mathrm{Fe} / \mathrm{H}]$ with excitation potential $(\mathrm{EP}=\chi)$ and reduced $\mathrm{EW}(\mathrm{REW}=\log \mathrm{EW} / \lambda)$ are minimized while simultaneously minimizing the difference between the iron abundances obtained from Fe I lines and those from Fe II lines (see Fig. B.1). For this work we employed the spectral analysis code MOOG (Sneden 1973) and linearly interpolated onedimensional local thermodynamic equilibrium (LTE) Kurucz ODFNEW model atmospheres (Castelli \& Kurucz 2003) via the Qoyllur-quipu (or $q^{2}$ ) Python package ${ }^{7}$ (Ramírez et al. 2014).

The iron line list, as well as the atomic parameters (EP and oscillator strengths, $\log g f$ ), were compiled from da Silva et al. (2011). The list includes $72 \mathrm{Fe} \mathrm{I}$ and $12 \mathrm{Fe}$ II lines in the range $\approx 4080-6752 \AA$, and EWs were manually measured using the splot task in IRAF. Since weak lines are the most sensitive to changes in the fundamental parameters, we selected only those lines with EWs $<100 \mathrm{~m} \AA$. Lines giving abundances departing $\pm 3 \sigma$ from the average were removed, and the fundamental parameters were recomputed ${ }^{8}$. We adopted the solar abundances from Asplund et al. (2009).

The resulting fundamental parameters, in both cases consistent with those of evolved stars (see Sect. 5.1), were $T_{\text {eff }}=4965 \pm$ $48 \mathrm{~K}, \log g=3.58 \pm 0.08 \mathrm{dex},[\mathrm{Fe} / \mathrm{H}]=0.22 \pm 0.04 \mathrm{dex}$, and $v_{\text {micro }}=1.12 \pm 0.09 \mathrm{~km} \mathrm{~s}^{-1}$ for Kepler- 278 and $T_{\text {eff }}=5038 \pm 24 \mathrm{~K}$, $\log g=3.62 \pm 0.05 \mathrm{dex},[\mathrm{Fe} / \mathrm{H}]=0.04 \pm 0.02 \mathrm{dex}$, and $v_{\text {micro }}=$ $0.93 \pm 0.06 \mathrm{~km} \mathrm{~s}^{-1}$ for Kepler-391. Here, the errors correspond to intrinsic uncertainties of the technique which are based on the scatter of the individual iron abundances from each individual line, the standard deviations in the slopes of the least-squares fits of iron abundances with REW, excitation, and ionization potential (see, e.g., Gonzalez \& Vanture 1998).

We checked for possible differences between the atmospheric parameters derived from the interpolated Kurucz's atmosphere models, obtained via $q^{2}$, and those explicitly calculated (i.e., non-interpolated). For the latter, we employed the FUNDPAR program $^{9}$ (Saffe 2011), that also derives atmospheric parameters based on the spectroscopic equilibrium procedure via the MOOG code but uses explicitly 1D LTE Kurucz's model atmospheres computed with ATLAS9 and ODFNEW opacities (Castelli \& Kurucz 2003). We find that the differences in the atmospheric parameters derived from these two set of models (in the sense interpolated - calculated) are very small: $\Delta T_{\text {eff }}=27 \mathrm{~K}, \Delta \log g=$ -0.02 dex, $\Delta[\mathrm{Fe} / \mathrm{H}]=-0.03 \mathrm{dex}, \Delta v_{\text {micro }}=-0.01 \mathrm{~km} \mathrm{~s}^{-1}$, and $\Delta T_{\text {eff }}=14 \mathrm{~K}, \Delta \log g=0.02 \mathrm{dex}, \Delta[\mathrm{Fe} / \mathrm{H}]=0.03 \mathrm{dex}, \Delta v_{\text {micro }}=$ $0.01 \mathrm{~km} \mathrm{~s}^{-1}$, for Kepler-278 and Kepler-391, respectively.

For consistency, we also computed the atmospheric parameters of both Kepler stars using MARCS model atmospheres (Gustafsson et al. 2008) instead of those from Kurucz's ODFNEW grid via the $q^{2}$ program. Although the MARCS grid that $q^{2}$ employs includes spherically-symmetric models, which are somewhat more realistic representations of evolved star atmospheres, we find that the dependency on the choice of model atmosphere grid is very weak. For both stars, the parameters derived from MARCS models are fully consistent with those computed with Kurucz model atmospheres, obtaining the following differences in the fundamental parameters

\footnotetext{
7 https://github.com/astroChasqui/q2

8 Final parameters for Kepler-278 were computed from 50 lines of Fe I and 9 of Fe II, whilst for Kepler-391 we employed 56 lines of Fe I and 8 lines of Fe II.

9 https://sites.google.com/site/saffecarlos/fundpar
}

(Kurucz-MARCS): $\Delta T_{\text {eff }}=20 \mathrm{~K}, \Delta \log g=0.01 \mathrm{dex}, \Delta[\mathrm{Fe} / \mathrm{H}]=$ -0.02 dex, $\Delta v_{\text {micro }}=-0.01 \mathrm{~km} \mathrm{~s}^{-1}$, and $\Delta T_{\text {eff }}=26 \mathrm{~K}, \Delta \log g=$ $0.02 \mathrm{dex}, \Delta[\mathrm{Fe} / \mathrm{H}]=0.02 \mathrm{dex}, \Delta v_{\text {micro }}=-0.01 \mathrm{~km} \mathrm{~s}^{-1}$, for Kepler-278 and Kepler-391, respectively. These results are in good agreement with those found in other evolved stars studies (e.g., Ramírez \& Allende Prieto 2011; Carlberg et al. 2012; Jofré et al. 2015b).

Regarding 3D or non-LTE effects, several studies show that these effects are noticeable only in warm $\left(T_{\text {eff }}>6000 \mathrm{~K}\right)$ and for very metal-poor evolved stars (e.g., Mashonkina et al. 2010; Lind et al. 2012). Since our stars have cooler temperatures and they are not very metal-poor, 3D and non-LTE effects should not compromise our results.

Additionally, we derived projected rotational velocities $(v \sin i)$ based on the spectral synthesis of six relatively isolated iron lines, following the procedure of Carlberg et al. (2012) and we adopted the calibration of Hekker \& Meléndez (2007) to determine the macroturbulence velocity, $v_{\text {macro }}$. We find both objects are slow rotators: $v \sin i=2.50 \pm 0.65 \mathrm{~km} \mathrm{~s}^{-1}$ and $v \sin i=$ $2.70 \pm 0.70 \mathrm{~km} \mathrm{~s}^{-1}$ for Kepler-278 and Kepler-391, respectively. These results are in excellent agreement with previous estimations computed from TRES and HIRES spectra (Buchhave et al. 2012; Huber et al. 2013; Petigura et al. 2017), and also with the expected velocities for similar evolved stars (e.g., de Medeiros et al. 1996).

\subsubsection{Consistency checks on $T_{\text {eff }}$ and $\log g$}

Fundamental atmospheric parameters, especially $T_{\text {eff }}$ and $\log g$, have a great impact on the stellar mass and radius determination and, ultimately, on the resulting planetary properties. Therefore, we present a set of consistency checks on our spectroscopically established $T_{\text {eff }}$ and $\log g$ values to determine their reliability and external accuracy (Sousa et al. 2011).

We performed the following checks on the spectroscopic effective temperatures:

Photometric estimate. We computed photometric effective temperatures using the metallicity-dependent $T_{\text {eff }}$-color calibrations from Casagrande et al. (2010). Based on available photometric data (see Table 1$)$, we calculated $(B-V),(V-J)$, $(V-H),\left(V-K_{S}\right)$, and $\left(J-K_{S}\right)$ colors. Magnitudes were corrected for extinction using the tables from Arenou et al. (1992) and adopting the extinction ratios, $k=E($ color $) / E(B-V)$, from Ramírez \& Meléndez (2005). Using our [Fe/H] values, we obtained average effective temperatures of $T_{\text {eff }}=4939 \pm$ $60 \mathrm{~K}$ and $T_{\text {eff }}=4997 \pm 80 \mathrm{~K}$ for Kepler-278 and Kepler-391, respectively, which in both cases are in good agreement with our spectroscopic $T_{\text {eff }}$ determinations.

Equivalent width line strength ratios. We used the $T_{\text {eff }}-\mathrm{LR}^{10}$ code that relies on the calibration between $T_{\text {eff }}$ and 433 line EWs ratios obtained by Sousa et al. (2010) analyzing 451 FGK dwarf stars. The EWs line ratios are built from 171 spectral lines of different chemical elements including $\mathrm{Fe}$, $\mathrm{Na}, \mathrm{Si}, \mathrm{Sc}, \mathrm{Cr}, \mathrm{Co}, \mathrm{Ti}, \mathrm{V}, \mathrm{Ni}$, and Co. In Fig. 1 we mark some of the doublets used for both stars. The 433 line ratios are build from a list of 171 lines (Sousa et al. 2010) whose EWs were measured automatically using the upgraded version of the code ARES $^{11}$ (Sousa et al. 2015). Of the 433 EWs ratios included in the list of Sousa et al. (2010) to build the $T_{\text {eff }}$-LR calibration, our

\footnotetext{
10 http://www.astro.up.pt/ sousasag/ares/line_ ratiopick2.php

11 http://www .astro.up.pt/ sousasag/ares/
} 
Table 1. Stellar properties of Kepler-278 and Kepler-391.

\begin{tabular}{|c|c|c|c|}
\hline Parameter & Kepler-278 & Kepler-391 & Source \\
\hline \multicolumn{4}{|c|}{ Astrometry } \\
\hline Right Ascension, RA & $19: 20: 25.73$ & $19: 22: 29.23$ & Gaia DR2 \\
\hline Declination, Dec & $+38: 42: 08.03$ & $+51: 03: 26.32$ & Gaia DR2 \\
\hline Proper motion in RA, $\mu_{\alpha}$ [mas $\mathrm{yr}^{-1}$ ] & $2.637 \pm 0.047$ & $10.3 \pm 0.034$ & Gaia DR2 \\
\hline Proper motion in Dec, $\mu_{\delta}$ [mas $\left.\mathrm{yr}^{-1}\right]$ & $8.95 \pm 0.052$ & $7.585 \pm 0.036$ & Gaia DR2 \\
\hline Parallax, $\pi$ [mas] ${ }^{(a)}$ & $2.146 \pm 0.119$ & $1.040 \pm 0.112$ & Gaia DR2 \\
\hline \multicolumn{4}{|c|}{ Kinematics and position } \\
\hline Barycentric radial velocity, $\mathrm{RV}\left[\mathrm{km} \mathrm{s}^{-1}\right]$ & $-46.98 \pm 0.04$ & $21.59 \pm 0.05$ & This work \\
\hline Distance, $d[\mathrm{pc}]^{(b)}$ & $465.98 \pm 25$ & $963 \pm 103$ & This work \\
\hline Space velocity component, $U\left[\mathrm{~km} \mathrm{~s}^{-1}\right]$ & $24.56 \pm 0.60$ & $36.33 \pm 2.36$ & This work \\
\hline Space velocity component, $V\left[\mathrm{~km} \mathrm{~s}^{-1}\right]$ & $-32.24 \pm 0.19$ & $40.43 \pm 0.71$ & This work \\
\hline Space velocity component, $W\left[\mathrm{~km} \mathrm{~s}^{-1}\right]$ & $1.26 \pm 0.15$ & $-14.67 \pm 1.35$ & This work \\
\hline Cartesian Galactic coordinate, $X[\mathrm{pc}]$ & $430.4 \pm 4.9$ & $916.8 \pm 10.5$ & This work \\
\hline Cartesian Galactic coordinate, $Y$ [pc] & $8349.9 \pm 96.1$ & $8379.3 \pm 96.4$ & This work \\
\hline Cartesian Galactic coordinate, $Z$ [pc] & $91.8 \pm 1.1$ & $268.8 \pm 3.1$ & This work \\
\hline \multicolumn{4}{|c|}{ Photometry $^{(c)}$} \\
\hline Kep [mag] & 11.584 & 13.007 & KIC \\
\hline$G$ [mag] & $11.530 \pm 0.003$ & $12.984 \pm 0.001$ & Gaia DR2 \\
\hline$B_{\mathrm{P}}[\mathrm{mag}]$ & $12.068 \pm 0.001$ & $13.492 \pm 0.002$ & Gaia DR2 \\
\hline$R_{\mathrm{P}}[\mathrm{mag}]$ & $10.873 \pm 0.001$ & $12.342 \pm 0.001$ & Gaia DR2 \\
\hline$B$ [mag] & $12.79 \pm 0.24$ & $14.075 \pm 0.018$ & APASS \\
\hline$V[\mathrm{mag}]$ & $11.818 \pm 0.14$ & $13.203 \pm 0.015$ & APASS \\
\hline$g[\mathrm{mag}]$ & 12.328 & $13.599 \pm 0.003$ & PAN-STARRS \\
\hline$r$ [mag] & 11.644 & 13.206 & PAN-STARRS \\
\hline$i[\mathrm{mag}]$ & 11.338 & 12.892 & PAN-STARRS \\
\hline$z[\mathrm{mag}]$ & 11.163 & 12.713 & PAN-STARRS \\
\hline$y[\mathrm{mag}]$ & 11.081 & $12.535 \pm 0.005$ & PAN-STARRS \\
\hline$J[\mathrm{mag}]$ & $10.047 \pm 0.021$ & $11.564 \pm 0.025$ & 2MASS \\
\hline$H[\mathrm{mag}]$ & $9.581 \pm 0.018$ & $11.072 \pm 0.027$ & 2MASS \\
\hline$K s[\mathrm{mag}]$ & $9.465 \pm 0.018$ & $10.97 \pm 0.025$ & 2MASS \\
\hline$W 1[\mathrm{mag}]$ & $9.37 \pm 0.024$ & $10.916 \pm 0.024$ & ALLWISE \\
\hline$W 2[\mathrm{mag}]$ & $9.456 \pm 0.02$ & $11.001 \pm 0.02$ & ALLWISE \\
\hline$W 3$ [mag] & $9.285 \pm 0.025$ & $10.991 \pm 0.057$ & ALLWISE \\
\hline$W 4[\mathrm{mag}]$ & $8.57 \pm 0.207$ & $<9.365$ & ALLWISE \\
\hline \multicolumn{4}{|c|}{ Fundamental and physical parameters } \\
\hline Effective temperature, $T_{\text {eff }}[\mathrm{K}]$ & $4965 \pm 54$ & $5038 \pm 57$ & This work \\
\hline Surface gravity, $\log g$ [cgs] & $3.58 \pm 0.095$ & $3.62 \pm 0.05$ & This work \\
\hline Metallicity, $[\mathrm{Fe} / \mathrm{H}]$ & $0.22 \pm 0.04$ & $0.04 \pm 0.02$ & This work \\
\hline Microturbulent velocity, $v_{\text {micro }}\left[\mathrm{km} \mathrm{s}^{-1}\right]$ & $1.12 \pm 0.09$ & $0.92 \pm 0.06$ & This work \\
\hline Macroturbulent velocity, $v_{\text {macro }}\left[\mathrm{km} \mathrm{s}^{-1}\right]$ & $3.54 \pm 1.32$ & $3.72 \pm 1.70$ & This work \\
\hline Rotational velocity, $v \sin i\left[\mathrm{~km} \mathrm{~s}^{-1}\right]$ & $2.50 \pm 0.67$ & $2.60 \pm 0.70$ & This work \\
\hline Spectral type, ST & K2 III-IV & K2 III-IV & This work \\
\hline Activity index $\log \left(R_{\mathrm{HK}}^{\prime}\right)_{\text {CaII-IRT }}$ & $-5.31 \pm 0.04$ & $-5.14 \pm 0.04$ & This work \\
\hline Mass, $M_{\star}\left[M_{\odot}\right]$ & $1.227 \pm 0.061$ & $1.270 \pm 0.081$ & This work \\
\hline Radius, $R_{\star}\left[R_{\odot}\right]$ & $2.861 \pm 0.060$ & $2.879 \pm 0.318$ & This work \\
\hline Age, $\tau_{\star}[\mathrm{Gyr}]$ & $5.761 \pm 1.019$ & $4.365 \pm 0.899$ & This work \\
\hline Density, $\rho_{\star}\left[\mathrm{g} \mathrm{cm}^{-3}\right]^{(d)}$ & $0.074 \pm 0.005$ & $0.073 \pm 0.006$ & This work \\
\hline Luminosity, $L_{\star}\left[L_{\odot}\right]^{(e)}$ & $4.46 \pm 0.57$ & $4.60 \pm 1.34$ & This work \\
\hline \multicolumn{4}{|c|}{ Asteroseismic properties } \\
\hline Frequency separation, $\Delta v[\mu \mathrm{Hz}]$ & $30.63 \pm 0.20$ & - & Huber et al. (2013) \\
\hline Frequency of maximum oscillation power, $v_{\max }[\mu \mathrm{Hz}]$ & $500.7 \pm 7.0$ & - & Huber et al. (2013) \\
\hline
\end{tabular}

Notes. ${ }^{(a)}$ Values have been corrected for the $-82 \pm 33 \mu$ arcsec offset found by Stassun \& Torres (2018). ${ }^{(b)}$ Derived from the corrected Gaia DR2 parallaxes (Gaia Collaboration 2018). To compute the errors on the distances, we have quadratically included 0.1 mas to the parallaxes uncertainties to account for systematic errors of Gaia's astrometry (Luri et al. 2018). ${ }^{(c)}$ We assume $1 \%$ of error when no errors are available in the catalogs. ${ }^{(d)}$ Calculated from the derived stellar radius and mass. ${ }^{\left({ }^{e}\right)}$ Calculated using the relation $\left(\frac{L_{\star}}{L_{\odot}}\right)=\left(\frac{R_{\star}}{R_{\odot}}\right)^{2}\left(\frac{T_{\text {eff, },}}{T_{\text {eff, }}}\right)^{4}$. 

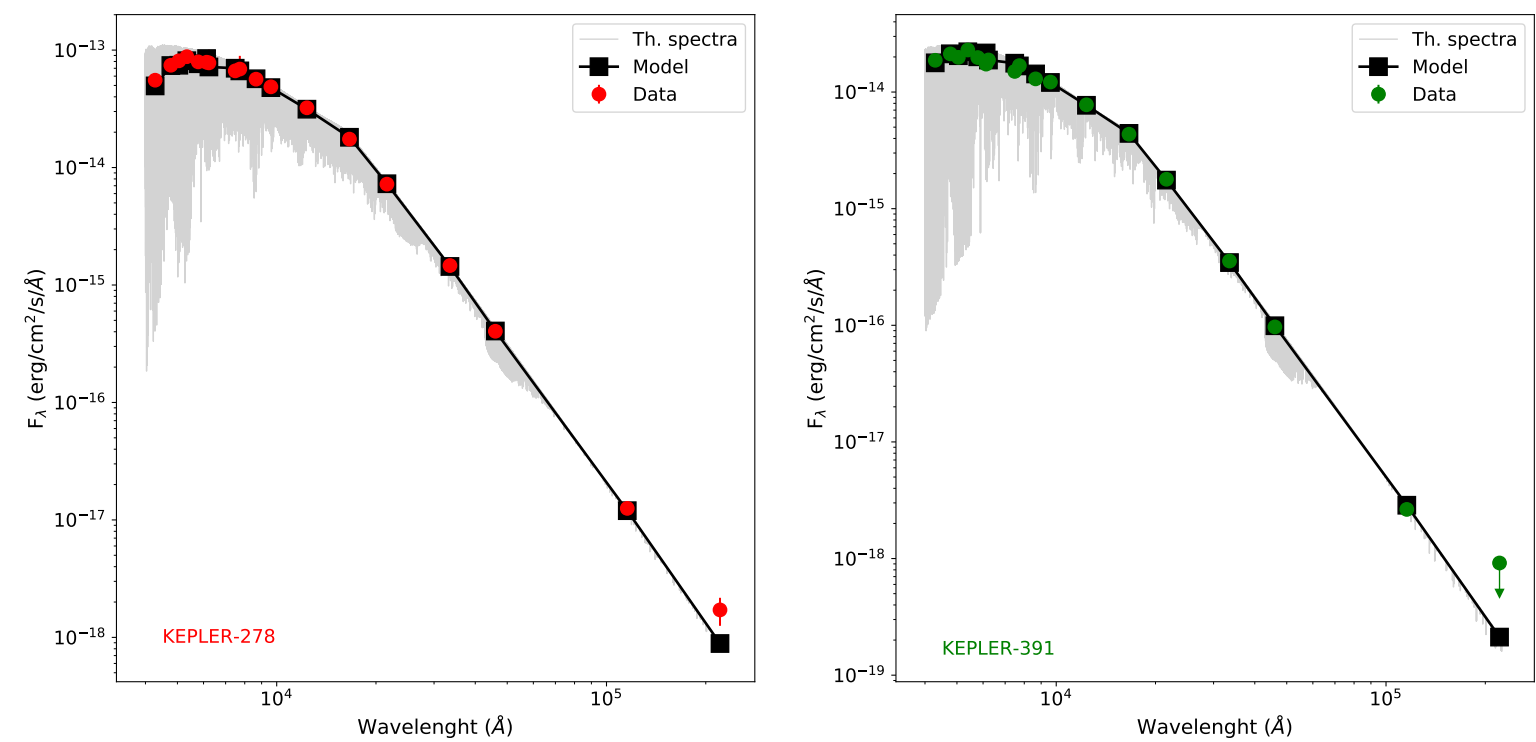

Fig. 2. Spectral energy distributions of Kepler-278 (left) and Kepler-391 (right). The black squares and black solid lines are the best fitting models, circles (red and green) mark the observed fluxes from the optical and infrared magnitudes listed in Table 1. Grey solid lines are the best-fit synthetic spectra.

final $T_{\text {eff }}$ values were computed from 376 EWs ratios for Kepler278 and from 368 for Kepler-391. This is because some of the EWs ratios (57 for Kepler-278 and 65 for Kepler-391) provided a temperature departing more than $2 \sigma$ from the average $T_{\text {eff }}$. For the final number of EWs ratios, we obtained $T_{\text {eff }}=4950 \pm 55 \mathrm{~K}$ for Kepler-278 and $T_{\text {eff }}=5070 \pm 50 \mathrm{~K}$ for Kepler-391. Even though the $T_{\text {eff }}$-LR calibration was built using only dwarfs, the resulting values for our evolved stars are consistent, within the error bars, with our spectroscopic determinations.

Spectral energy distribution. We obtained $T_{\text {eff }}$ from the analysis of the Spectral Energy Distribution (SED) based on the latest version of the Virtual Observatory SED Analyzer ${ }^{12}$ (VOSA, Bayo et al. 2008). We constructed the SEDs of Kepler-278 and Kepler-391 from broadband photometry, including magnitudes from AAVSO Photometric All-Sky Survey (APASS $^{13}$; Henden et al. 2015), Panoramic Survey Telescope and Rapid Response System (PAN-STARRS; Hodapp et al. 2004; Chambers et al. 2016), 2-Micron All-Sky Survey (2MASS; Skrutskie et al. 2006), Gaia DR2 (Gaia Collaboration 2018), Kepler (Borucki et al. 2010), and Wide-field Infrared Survey Explorer (WISE; Wright et al. 2010). Table 1 summarizes this information. We modeled the data using BT-NextGen stellar model atmospheres (Allard et al. 2012) from a Chi-square fit in order to compute the expected values of $T_{\text {eff }}$, surface gravity, metallicity, extinction, and a proportionality factor. The effective temperatures computed from VOSA are $T_{\text {eff }}=5000 \pm 50 \mathrm{~K}$ and $T_{\text {eff }}=5100 \pm 60 \mathrm{~K}$ for Kepler-278 and Kepler-391, respectively, which once again agree reasonably well with our spectroscopic estimates. The SEDs for both stars are shown in Fig. 2. From these figures, it can be noticed that Kepler-278 might exhibit a small infrared excess which is particularly notable at the $22 \mu \mathrm{m}$ WISE W4 bandpass. However, using the test presented in Rebull et al. (2015), we find that the W4-excess would not be significant. Although Kepler-391 also seems to show an IR excess, in this case, the W4 magnitude is only an upper limit value.

Given the good agreement between the effective temperatures of Kepler-278 and Kepler-391 estimated from GRACES

\footnotetext{
12 http://svo2.cab.inta-csic.es/theory/vosa/

13 http://aavso.org/apass
}

spectra and those derived from the approaches detailed above (see left panel of Fig. 3), we can conclude that the first ones are reliable and, therefore, we adopt them as our final values to compute the chemical abundances and physical stellar and planetary parameters in the next sections.

On the other hand, for the surface gravities, we carried out the following tests:

Surface gravity from $\mathrm{Mg}$ ı $b, \mathrm{Na}$ । $\mathrm{D}$, and $\mathrm{Ca}$ lines. We obtained $\log g$ values from the spectral synthesis of the wings of several strong features using the iSpec spectral analysis tool ${ }^{14}$ (Blanco-Cuaresma et al. 2014), following a similar method as described in Bruntt et al. (2010), Ramírez et al. (2011), and Doyle et al. (2017). The lines analyzed include Mg I b ( $\lambda 5180 \AA$ A), Na I D $(\lambda 5889,5895 \AA)$, and Ca I $(\lambda 6122,6162,6439 \AA)$ from which we obtained an average value of $\log g=3.66 \pm 0.10$ dex for Kepler-278 and $\log g=3.70 \pm 0.08$ dex for Kepler-391. Both values are in agreement, within the errors, with the estimates found from the spectroscopic equilibrium.

Asteroseismic gravity. Asteroseismic parameters (such as the maximum frequency, $v_{\max }$ ), obtained from high-precision photometry (e.g., Kepler or CoRoT), can be combined with a $T_{\text {eff }}$ estimation to provide very accurate surface gravities (e.g., Gai et al. 2011; Morel \& Miglio 2012; Hekker et al. 2013). Using asteroseismic data from Huber et al. (2013) and the scaling relation of Brown et al. (1991):

$\log g_{\text {seis }}=\log g_{\odot}+\log \left(\frac{v_{\text {max }}}{v_{\text {max }, \odot}}\right)+\frac{1}{2} \log \left(\frac{T_{\text {eff }}}{T_{\text {eff }, \odot}}\right)$

where $v_{\text {max }, \odot}=3090 \mu \mathrm{Hz}, T_{\text {eff }, \odot}=5777 \mathrm{~K}$ and $\log g_{\odot}=4.44 \mathrm{dex}$, we find $\log g_{\text {seis }}=3.61 \pm 0.02$ dex for Kepler-278, which is in good agreement with the spectroscopic value. We could not perform this check on Kepler-391 because, unfortunately, there is no asteroseismic information available for this star. The reason for this is that Kepler-391 does not have Kepler short-cadence data and, moreover given its $\log g$, the $v_{\max }$ value for this star is expected to be above the Nyquist frequency for the long-cadence data (e.g., Chaplin et al. 2014).

${ }^{14}$ https://www.blancocuaresma.com/s/iSpec 

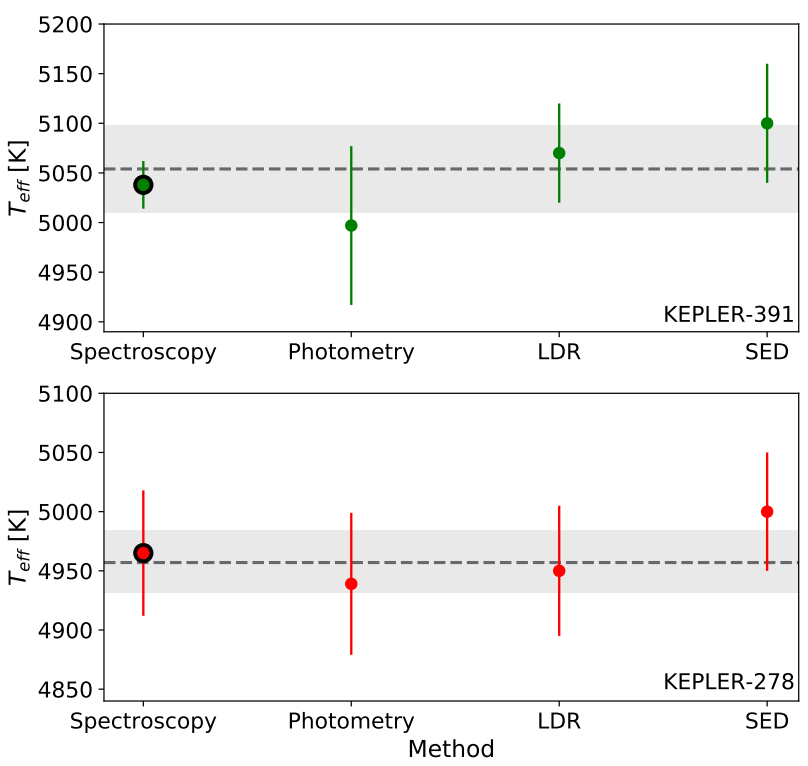
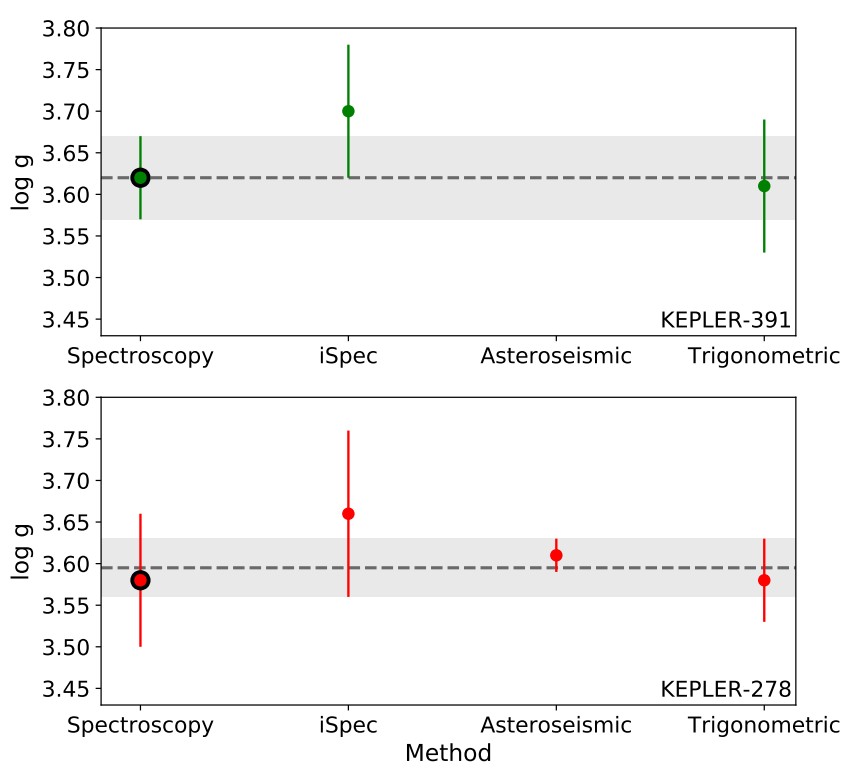

Fig. 3. Stellar temperatures (left) and surface gravity values (right) obtained by the different consistency checks detailed in Sect. 3.1.1. Dashed lines mark the median values and the shaded areas indicate the standard deviations. The adopted spectroscopic $T_{\text {eff }}$ and $\log g$ values for Kepler-278 and Kepler-391 are showed with black edge-color circles.

Trigonometric gravities. We employed the 1.3 version of the PARAM web interface ${ }^{15}$ that performs a Bayesian estimation of stellar parameters (da Silva et al. 2006; Miglio et al. 2013) based on PARSEC isochrones (Bressan et al. 2012). As input we used $V$ magnitudes, Gaia DR2 parallaxes (Gaia Collaboration 2018) and our spectroscopic $T_{\text {eff }}$ and $[\mathrm{Fe} / \mathrm{H}]$ values. In perfect agreement with our previous estimations, PARAM returned $\log g=$ $3.59 \pm 0.05$ dex for Kepler-278 and $\log g=3.61 \pm 0.08$ dex for Kepler-391. Similar results are obtained using the $q^{2}$ program from which we computed $\log g=3.63 \pm 0.06$ dex for Kepler-278 and $\log g=3.60 \pm 0.05$ dex for Kepler-391.

Similar to the results obtained for $T_{\text {eff }}$, we find that the $\log g$ values determined with other independent methods are in good agreement with the ones determined in this work via ionization equilibrium for both stars (see right panel of Fig. 3), and hence, ensuring their reliability.

We also tested the effects on $T_{\text {eff }}$ and $[\mathrm{Fe} / \mathrm{H}]$ of fixing the surface gravity to an external accurate value. Recently, it has been suggested the existence of a strong degeneracy between $T_{\text {eff }}$, $[\mathrm{Fe} / \mathrm{H}]$, and $\log g$ when solving for all three quantities simultaneously, especially when methods based on spectral synthesis are used (Torres et al. 2012). To avoid this degeneracy and improve stellar parameters, it has been pointed out that fixing $\log g$ to an external accurate value (e.g., from asteroseismic data or transits) improves the final stellar parameters. However, it has also been noticed that fixing $\log g$ to an external value has no significant effect on the temperatures or metallicities when working with methods based on EWs, and then it is sufficient to use the unconstrained values (Mortier et al. 2014; Doyle et al. 2017).

We checked these results for Kepler-278 by recomputing their fundamental parameters with $\log g$ fixed to the accurate value estimated from asteroseismology ${ }^{16}$, obtaining $T_{\text {eff }}=$ $4967 \pm 49 \mathrm{~K}$ and $[\mathrm{Fe} / \mathrm{H}]=0.24 \pm 0.04 \mathrm{~K}$, which represent a difference of only $2 \mathrm{~K}$ and 0.02 dex when compared to the parameters obtained with no constraints on $\log g$. Therefore, in

\footnotetext{
15 http://stev.oapd.inaf.it/cgi-bin/param_1.3

${ }^{16} \log g$ from transits might be less accurate for shallower transits, low $\mathrm{S} / \mathrm{N}$ light curves or planets in eccentric orbits (Huber et al. 2013).
}

agreement with the results of Mortier et al. (2014) and Doyle et al. (2017), we find that $T_{\text {eff }}$ and $[\mathrm{Fe} / \mathrm{H}]$ are not significantly altered when $\log g$ is fixed and, hence, in the next sections we adopt the unconstrained set of fundamental stellar parameters.

\subsubsection{Formal errors}

In addition to the internal precision errors for $T_{\text {eff }}$ and $\log g$ provided in Sect. 3.1, we also computed systematic (or accuracy) errors for these parameters following Sousa et al. (2011). We derived an estimation of the systematic error in $T_{\text {eff }}$ and $\log g$ by comparing the results obtained from the excitation and ionization equilibrium with those derived with the other independent methods in Sect. 3.1.1. For $T_{\text {eff }}$, we obtained a mean difference of $1 \pm 25 \mathrm{~K}$ and $22 \pm 51 \mathrm{~K}$ for Kepler-278 and Kepler-391. Therefore, we can assume a systematic error in $T_{\text {eff }}$ of 25 and $51 \mathrm{~K}$ for Kepler-278 and Kepler-391, respectively, which are consistent with the average systematic error obtained recently for a large sample of subgiant stars with planets by Ghezzi et al. (2018).

In the case of surface gravity, the comparison between the $\log g$ values obtained via ionization balance and those derived with the other techniques revealed a mean difference of $0.04 \pm$ 0.04 dex and $0.05 \pm 0.04$ dex for Kepler-278 and Kepler-391, respectively. Then, we adopted 0.04 dex as the systematic error in $\log g$ for Kepler-278 and Kepler-391.

The formal errors in the spectroscopic $T_{\text {eff }}$ and $\log g$ are taken as the quadratic sum of the intrinsic and systematic errors. The formal error for $[\mathrm{Fe} / \mathrm{H}]$ is computed by adding in quadrature the intrinsic error, given by the scatter of the individual line-to-line iron abundances, and the errors introduced by propagating our formal uncertainties in the other atmospheric parameters. The final atmospheric parameters along with their formal errors are summarized in the fourth block of Table 1. An additional source of error in the spectroscopic parameters, not taken into account in this work, could come from the use of classical solar-scaled opacities instead of non-solar-scaled opacities that could amount up to $26 \mathrm{~K}, 0.05 \mathrm{dex}$, and $0.02 \mathrm{dex}$ in $T_{\text {eff }}, \log g$, and $[\mathrm{Fe} / \mathrm{H}]$, respectively (Saffe et al. 2018). 

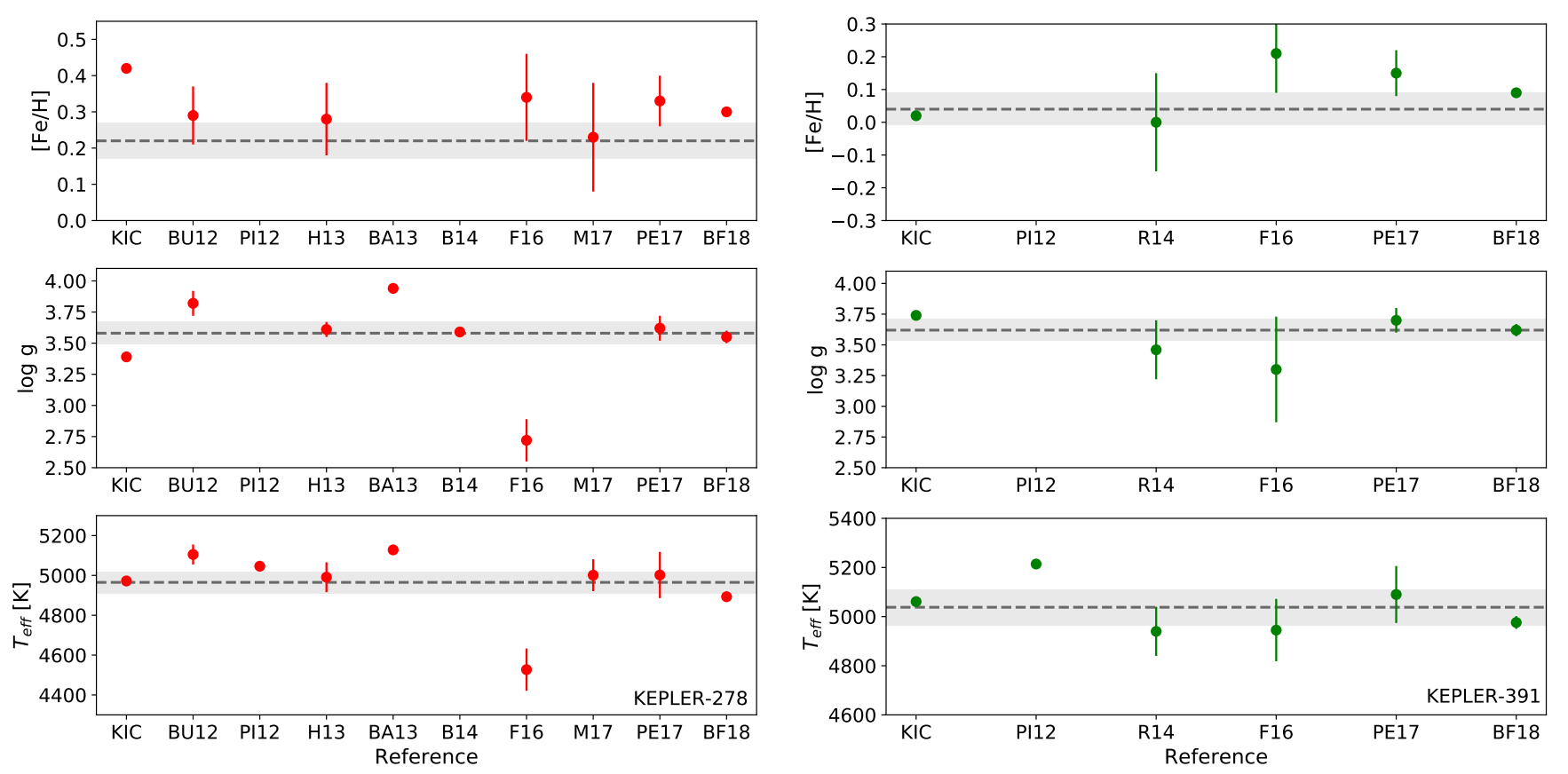

Fig. 4. Comparison of our spectroscopic $T_{\text {eff }}, \log g$, and [Fe/H] values (dashed lines) for Kepler-278 (left) and Kepler-391 (right) with those reported by the Kepler Input Catalogue (Kepler Mission Team 2009, KIC), Buchhave et al. (2012, BU12), Pinsonneault et al. (2012, PI12), Huber et al. (2013, H13), Batalha et al. (2013, BA13), Rowe et al. (2014, R14), Bastien et al. (2014), Frasca et al. (2016, F16), Mathur et al. (2017, M17), Petigura et al. (2017, PE17), and Brewer \& Fischer (2018, BF18). Grey shaded areas indicate the formal error in our results as detailed in Sect. 3.1.2.

\subsubsection{Comparison with previous works}

As a final external validation test on the reliability of our estimations, we compared our results with those previously derived based on different methods and data quality. Figure 4 shows our $T_{\text {eff }}, \log g$, and $[\mathrm{Fe} / \mathrm{H}]$ estimations in comparison with those of the Kepler Input Catalog (KIC, Kepler Mission Team 2009), Buchhave et al. (2012, BU12 hereafter), Pinsonneault et al. (2012, PI12 hereafter), Huber et al. (2013, H13 hereafter), Batalha et al. (2013, BA13 hereafter), Rowe et al. (2014, R14 hereafter), Bastien et al. (2014, B14 hereafter), Frasca et al. (2016, F16 hereafter), Mathur et al. (2017, M17 hereafter), Petigura et al. (2017, PE17 hereafter), and Brewer \& Fischer (2018, BF18 hereafter). In general, our estimations for both stars are in fair agreement with those from previous studies, although a few measurements deviate $\sim 2 \sigma$ from our values. Next we discuss the potential source of these discrepancies.

Although for Kepler-391 our results are in excellent agreement with those from the $\mathrm{KIC}^{17}$, for Kepler-278 the discrepancies are particularly significant for the surface gravity and $[\mathrm{Fe} / \mathrm{H}]$ values $(\Delta \log g=0.19$ dex, and $\Delta[\mathrm{Fe} / \mathrm{H}]=-0.2 \mathrm{dex})$. As we mentioned in the introduction, the limited accuracy of atmospheric parameters (in particular $\log g$ and $[\mathrm{Fe} / \mathrm{H}]$ ) based on broadband photometric calibrations only (Huber et al. 2014; Bruntt et al. 2012) might explain the discrepancies with our spectroscopic values. This also could be the origin for the differences with the effective temperatures derived by PI12, which are also based on photometric calibrations at fixed $[\mathrm{Fe} / \mathrm{H}]=-0.2 \mathrm{dex}$. Their temperatures are larger than our values by 84 and $176 \mathrm{~K}$ for Kepler-278 and Kepler-391, respectively.

From high-resolution reconnaissance spectra (i.e., with low $\mathrm{S} / \mathrm{N}$ ) obtained by the Kepler Follow-up Observing Program (FOP), BU12 reported fundamental parameters of the host stars of 226 small exoplanet candidates discovered by Kepler,

$\overline{17}$ No errors are provided in this catalog. including Kepler-278. Their results are obtained using the Stellar Parameter Classification (SPC) technique, which fits synthetic spectra to the observed data. Their results for Kepler- $278^{18}$, although within the error, are systematically larger than ours: $\Delta T_{\text {eff }}=-140 \mathrm{~K}, \Delta \log g=-0.24 \mathrm{dex}$, and $\Delta[\mathrm{Fe} / \mathrm{H}]=-0.07$ dex. Potential sources of this discrepancy include the substantial difference in the $\mathrm{S} / \mathrm{N}$ of the spectra used and the distinct analysis techniques. Moreover, as we mentioned before, techniques based on spectral synthesis are more sensitive to show strong systematic biases on $T_{\text {eff }}$ and $[\mathrm{Fe} / \mathrm{H}]$ when $\log g$ is unconstrained (Torres et al. 2012). This also could explain the large differences obtained in $T_{\text {eff }}\left(\Delta T_{\text {eff }}=163 \mathrm{~K}\right)$ and $\log g(\Delta \log g=0.16$ dex $)$ with the parameters determined by BA $13^{19}$ from spectral synthesis based on low $\mathrm{S} / \mathrm{N}$ and high resolution spectroscopy (Gautier et al. 2010).

The largest discrepancies, in both stars, are obtained with the results of F16 who determined stellar parameters from the spectral synthesis of LAMOST low resolution spectra (Wang et al. 1996). For Kepler-278 the differences are $\Delta T_{\text {eff }}=438 \mathrm{~K}$, $\Delta \log g=0.86 \mathrm{dex}$, and $\Delta[\mathrm{Fe} / \mathrm{H}]=-0.12 \mathrm{dex}$. For Kepler-391 the differences are a little bit smaller, but still the largest in comparison with any of the other studies: $\Delta T_{\text {eff }}=93 \mathrm{~K}, \Delta \log g=$ $0.32 \mathrm{dex}$, and $\Delta[\mathrm{Fe} / \mathrm{H}]=-0.17 \mathrm{dex}$. The most probable sources for these discrepancies are the quality of the spectra, especially resolution, and the analysis technique, in which $\log g$ values are not constrained (e.g., Doyle et al. 2017).

\subsection{Stellar activity indicators}

Following the procedure described in Mittag et al. (2013) for evolved stars, we characterized the chromospheric stellar activity of Kepler-278 and Kepler-391 from the fluxes of the Ca II H \& $\mathrm{K}$ lines centered at $\lambda 3968$ and $\lambda 3934 \AA$ via the $S$ and $\log \left(R_{\mathrm{HK}}^{\prime}\right)$

18 Obtained using a spectrum with $S / N \approx 45$.

${ }^{19}$ No estimation of $[\mathrm{Fe} / \mathrm{H}]$ nor errors in $T_{\text {eff }}$ and $\log g$ are reported. 

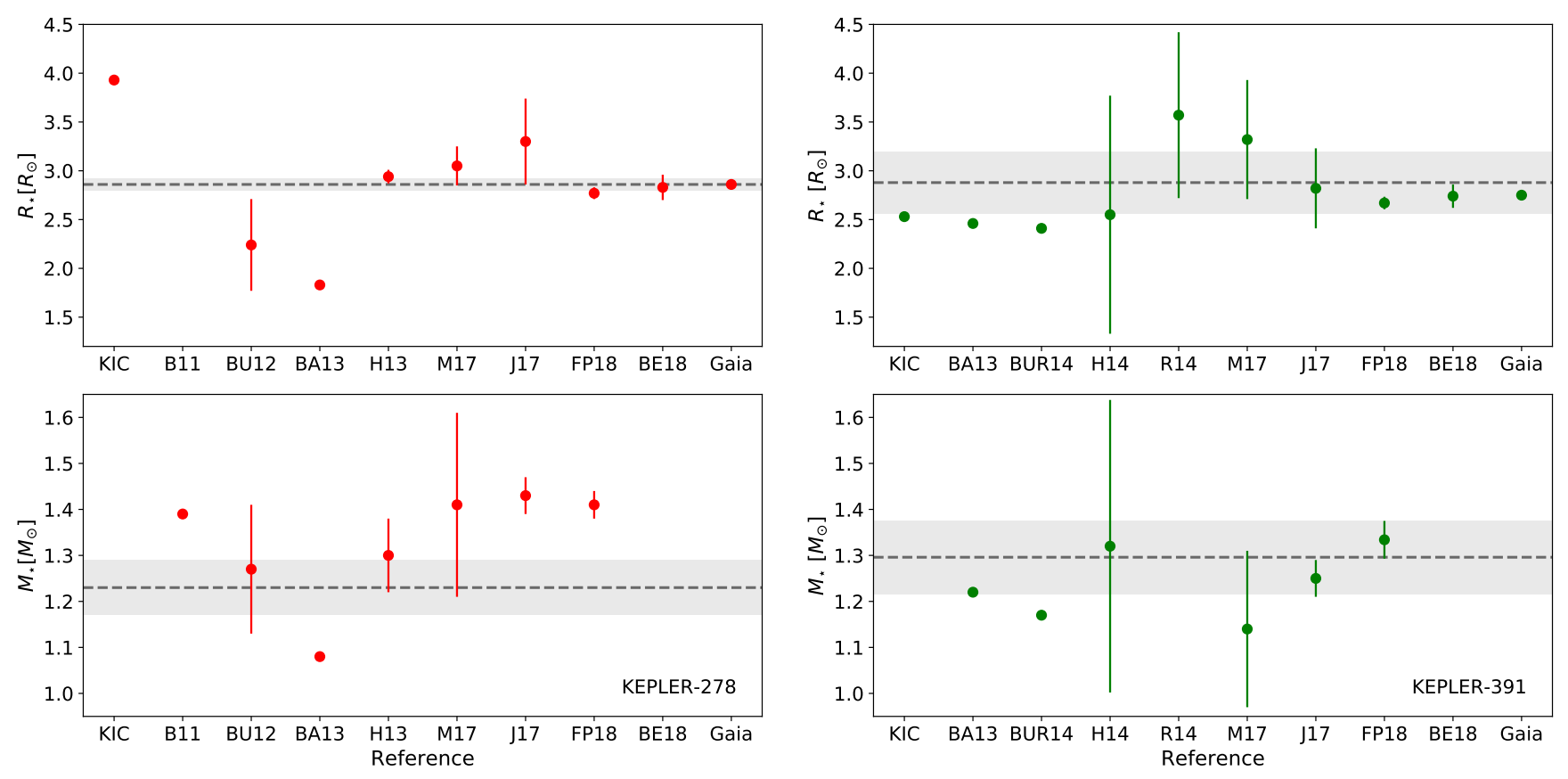

Fig. 5. Stellar masses and radii determined by different authors (filled circles) in comparison with those derived in this study (dashed lines) for Kepler-278 (left) and Kepler-391 (right). Grey shaded areas indicate $1 \sigma$ uncertainty in our results.

indicators (e.g., Baliunas et al. 1995; Lovis et al. 2011; Egeland et al. 2017). Since the wavelength coverage of the GRACES spectra does not include these lines, we used public Keck-HIRES spectra obtained by the California-Kepler Survey ${ }^{20}$ (CKS, Petigura et al. 2017). We obtained $S=0.11$ and $\log \left(R_{\mathrm{HK}}^{\prime}\right)=-5.10$ for Kepler-278, whilst for Kepler-391 we derived $S=0.12$ and $\log \left(R_{\mathrm{HK}}^{\prime}\right)=-5.14$. These values are in line with those found for the vast majority of subgiants, indicating low chromospheric activity (Isaacson \& Fischer 2010). Moreover, our derived $S$ and $\log \left(R_{\mathrm{HK}}^{\prime}\right)$ values are in good agreement with those computed recently by Brewer \& Fischer (2018), although their $\log \left(R_{\mathrm{HK}}^{\prime}\right)$ values were based on the definition of Noyes et al. (1984), which is only valid for main-sequence stars. Additionaly, following Isaacson \& Fischer (2010), we estimated a jitter of $4.33 \mathrm{~m} \mathrm{~s}^{-1}$ for Kepler-278 and $4.11 \mathrm{~m} \mathrm{~s}^{-1}$ for Kepler-391.

On the other hand, given that GRACES spectra include the infrared triplet lines of ionized calcium (Ca II-IRT) at 8498, 8542 and $8662 \AA$, we obtained information about stellar activity from these lines following the method outlined in Busà et al. (2007). In particular, we estimated $\log \left(R_{\mathrm{HK}}^{\prime}\right)=-5.31 \pm 0.04$ for Kepler-278 and $\log \left(R_{\mathrm{HK}}^{\prime}\right)=-5.14 \pm 0.04$ for Kepler-391, considering a $10 \%$-error in the stellar flux. Given that for Kepler-391 the $\log \left(R_{\mathrm{HK}}^{\prime}\right)$ values derived from HIRES and GRACES data are in good agreement, we consider that the difference in the results for this activity indicator for Kepler-278 could be caused mainly by a difference in the level of stellar activity. It would be worthwhile to analyze additional spectroscopic data in order to confirm the stellar variability that might be caused by stellar spots which already have been detected in their photometry (Van Eylen \& Albrecht 2015).

\subsection{Stellar mass, radius, and age}

We derived the stellar mass $M_{\star}$, radius $R_{\star}$, and age $\tau_{\star}$ of Kepler278 and Kepler-391 through the 1.3 version of the Bayesian

${ }^{20}$ Observations were taken on 2014 July 14 UT for Kepler-278 and on 2012 June 20 UT for Kepler-391. interface PARAM (da Silva et al. 2006) using PARSEC isochrones (Bressan et al. 2012). This applet also gives $\log g$, as mentioned in Sect. 3.1.1. As input, we provided our spectroscopic $T_{\text {eff }}$ and $[\mathrm{Fe} / \mathrm{H}]$, Gaia DR2 parallaxes (Gaia Collaboration 2018) corrected for the $82 \mu$ arcsec offset found by Stassun \& Torres (2018), and $\mathrm{V}$ dereddened magnitudes along with all the parameters' uncertainties. For Kepler-278, PARAM returned $M_{\star}=1.325 \pm$ $0.060 M_{\odot}, R_{\star}=2.973 \pm 0.152 R_{\odot}$, and $\tau_{\star}=4.466 \pm 0.630 \mathrm{Gyr}$, whilst for Kepler-391, we found $M_{\star}=1.296 \pm 0.080 M_{\odot}, R_{\star}=$ $2.879 \pm 0.318 R_{\odot}$, and $\tau_{\star}=4.365 \pm 0.899$ Gyr. The computed stellar masses and radii imply stellar densities of $\rho_{\star}=0.071 \pm$ $0.006 \mathrm{~g} \mathrm{~cm}^{-3}$ and $\rho_{\star}=0.077 \pm 0.011 \mathrm{~g} \mathrm{~cm}^{-3}$ for Kepler-278 and Kepler-391, respectively.

As another option, the asteroseismic quantities ( $\Delta v$ : large frequency separation and $v_{\max }$ : frequency of maximum oscillation power) can be used in PARAM as input parameters in replacement of $V$ magnitude and parallax. Using this option for Kepler-278, for which seismic information is available $\left(v_{\max }=500.7 \pm 7 \mu \mathrm{Hz}\right.$, $\Delta v=30.63 \pm 0.20 \mu \mathrm{Hz}$; Huber et al. 2013), PARAM returned $M_{\star}=1.227 \pm 0.061 M_{\odot}, R_{\star}=2.861 \pm 0.057 R_{\odot}, \tau_{\star}=5.761 \pm$ $1.019 \mathrm{Gyr}, \log g=3.606 \pm 0.006 \mathrm{dex}$, and from the values of $M_{\star}$ and $R_{\star}$ we derived $\rho_{\star}=0.074 \pm 0.005 \mathrm{~g} \mathrm{~cm}^{-3}$. All values are in excellent agreement, within $1 \sigma$, with those obtained with the first input. As final results, for Kepler-278 we adopted these values based on asteroseismic information and those based on DR2 Gaia parallaxes for Kepler-391. Independent techniques produced consistent results as can be seen in Appendix A.

Comparison with previous works. As a further check on our computed stellar physical parameters, we compared them with those reported in the literature. In Fig. 5, we show the comparison of our stellar masses and radii of Kepler-278 and Kepler-391 with those obtained by KIC, Borucki et al. (2011, B11 hereafter), BU12, Batalha et al. (2013, BA13 hereafter), H13, Huber et al. (2014, H14 hereafter), R14, M17, Johnson et al. (2017, J17 hereafter), Fulton \& Petigura (2018, FP18 hereafter), and Berger et al. (2018, BE18 hereafter). As can be noticed, there 
are significant discrepancies with some of the results of previous studies, mainly for Kepler-278.

The stellar radius reported in the KIC, $R_{\star}=3.93 R_{\odot}$, is $\sim 37 \%$ larger than our value. As we mentioned before, the origin of this discrepancy is likely caused by the non-negligible difference between fundamental parameters (see Sect. 3.1.3). In line with this difference in the stellar radius, Johnson et al. (2017) found that stellar radii in the KIC, based on broadband photometry only, have fractional uncertainties of $40 \%$.

Based on the $R_{\star}$ and $\log g$ values reported by KIC, B11 derived a stellar mass of $M_{\star}=1.39 M_{\odot}$ (no error provided) for Kepler-278, which is $13 \%$ larger than our value. Again, this is likely due to the limited accuracy of stellar parameters in the KIC.

BU12 used the $T_{\text {eff }}, \log g$, and $[\mathrm{Fe} / \mathrm{H}]$ determined via the SPC technique from the low $\mathrm{S} / \mathrm{N}$ reconnaissance spectra, in combination with a grid of Yonsei-Yale models to infer the stellar mass and radius of Kepler-278. Although the stellar mass reported by BU12 is in good agreement with our value, their radius is $\sim 22 \%$ smaller than our estimation. This discrepancy is likely originated by the large difference between our $T_{\text {eff }}$ and $\log g$ values and those from BU12 (see Sect. 3.1.3).

H13 determined $M_{\star}$ and $R_{\star}$ using the so called grid-based modeling, where atmospheric parameters $\left(T_{\text {eff }},[\mathrm{Fe} / \mathrm{H}]\right)$ and asteroseismic contraints are fitted to a grid of isochrones ${ }^{21}$. For Kepler-278, their estimations agree, within the errors, with our results.

BA13 employed $T_{\text {eff }}, \log g$, and $[\mathrm{Fe} / \mathrm{H}]$ derived from spectroscopy (for Kepler-278) or values compiled from KIC (for Kepler-391) as initial constraints to obtain stellar masses and radii, through Yonsei-Yale stellar evolution models. For Kepler391 they reported $M_{\star}=1.22 M_{\odot}$ and $R_{\star}=2.46 R_{\odot}$ (no errors provided), which are just below our $1 \sigma$ range. For Kepler-278, BA13 reported $M_{\star}=1.08 M_{\odot}$ and $R_{\star}=1.80 R_{\odot}$, which are $\sim 12$ and $\sim 36 \%$ smaller than our estimations. The origin of these discrepancies is probably related to differences between our fundamental parameters and those from BA13, especially for Kepler-278 (see Fig. 4).

BUR14 employed photometric $T_{\text {eff }}$ from PI12 and $\log g$ and $[\mathrm{Fe} / \mathrm{H}]$ values from the $\mathrm{KIC}$ as initial constraints to obtain stellar masses and radii via stellar evolution models as in BA13. For Kepler-391, they determined $M_{\star}=1.17 M_{\odot}$ and $R_{\star}=2.41 R_{\odot}$ (no errors provided), which are very similar to those from BA13 and are just outside the $1 \sigma$ region in our results. As before, the cause of these discrepancies is likely related to differences between our fundamental parameters, especially for the $T_{\text {eff }}$ (see Fig. 4).

For Kepler-391, R14 derived stellar parameters based on Yonsei-Yale models matching, using the atmospheric parameters derived from low S/N HIRES spectra as initial constraints. They estimated $R_{\star}=3.57 \pm 0.85 R_{\odot}$, which is $\sim 24 \%$ larger than our estimation. Curiously, they do not report the value for the stellar mass nor the age. However, they report an estimation for the stellar density of $\rho_{\star}=0.042 \mathrm{~g} \mathrm{~cm}^{-3}$, which combined with the stellar radius would imply a mass of $M_{\star}=1.35 M_{\odot}$. All the parameters agree with our results within the errors.

J17 used Dartmouth stellar evolution models to convert the spectroscopic properties $T_{\text {eff }}, \log g$, and $[\mathrm{Fe} / \mathrm{H}]$, compiled from $\mathrm{PE} 17$, into mass, radius, and age via the isochrones code (Morton 2015). For Kepler-391, their estimations agree well with

\footnotetext{
${ }^{21} \mathrm{H} 13$ used six different models: ASTEC (Christensen-Dalsgaard 2008), BaSTI (Pietrinferni et al. 2004), DSEP (Dotter et al. 2008), Padova (Marigo et al. 2008), Yonsei-Yale (Demarque et al. 2004), and YREC (Demarque et al. 2008).
}

our results. However, for Kepler-278, their derived stellar radius and mass values are $\sim 15 \%$ and $\sim 16 \%$ larger than our estimations. Recently, FP18 derived stellar radii for the CKS sample from the Stefan-Boltzmann law based on Gaia parallaxes, Kepler photometry, and spectroscopic temperatures compiled from PE17. In parallel, they also determined masses, radii, and ages via MIST isochrone grids (Choi et al. 2016) by providing $T_{\text {eff }}, \log g$, and [Fe/H] from PE17, along with $m_{K}$ 2MASS constraints through the isoclassify package (Huber et al. 2017). The agreement between the stellar radii they derived by different approaches is excellent both for Kepler-278 and Kepler-391. However, similarly to $\mathrm{J} 17$, we found a significant discrepancy in the mass values for Kepler- 278 of $\sim 15 \%$. Given that there is no significant difference between our fundamental parameters against those of PE17 and $\mathrm{J} 17$, the origin of the discrepancies in the stellar mass of Kepler278 is not clear. A possibility could be the use of different stellar models (e.g., MESA vs. PARSEC) to obtain the stellar parameters, although it is not evident why there are no differences for Kepler-391. Additionally, as demonstrated by Huber et al. (2019) for TOI-197, uncertainties in the stellar parameters can be underestimated by the use of single, instead of multiple, model grids that do not allow to take systematic errors into account.

Finally, our stellar radii are in perfect agreement with those provided by Gaia DR2 (Gaia Collaboration 2018), based on SED modeling, and those from BE18 derived by combining Gaia DR2 parallaxes with the DR25 Kepler Stellar Properties Catalog.

\subsection{Detailed chemical abundances}

\subsubsection{Analysis}

From the high-quality GRACES spectra obtained for Kepler-278 and Kepler-391, in addition to iron, we derived chemical abundances of five light ( $\mathrm{Li}, \mathrm{C}, \mathrm{N}, \mathrm{Na}, \mathrm{Al}$ ), eight iron-peak ( $\mathrm{Sc}, \mathrm{V}, \mathrm{Cr}$, $\mathrm{Mn}, \mathrm{Co}, \mathrm{Ni}, \mathrm{Cu}, \mathrm{Zn}$ ), six alpha (O, Mg, Si, S, Ca, Ti), and five heavy elements ( $\mathrm{Sr}, \mathrm{Y}, \mathrm{Zr}, \mathrm{Ba}, \mathrm{Ce})$. Abundances were computed using both EWs and spectrum synthesis analysis in combination with the LTE Kurucz model atmospheres previously calculated.

The abundances of $\mathrm{O}, \mathrm{Na}, \mathrm{Mg}, \mathrm{Al}, \mathrm{Si}, \mathrm{Ca}, \mathrm{Sc}, \mathrm{Ti}, \mathrm{V}, \mathrm{Cr}$, $\mathrm{Mn}, \mathrm{Co}, \mathrm{Ni}, \mathrm{Cu}, \mathrm{Zn}, \mathrm{Sr}, \mathrm{Y}, \mathrm{Zr}, \mathrm{Ba}$, and Ce were derived from the curve-of-growth approach employing the MOOG code (abfind driver) through the $q^{2}$ code. The EWs were measured carefully by hand using Gaussian profile fits with the task splot in IRAF. The line-list and atomic parameters for most of the elements were compiled from Neves et al. (2009), Adibekyan et al. (2015), Takeda et al. (2005), Chavero et al. (2010), Ramírez et al. (2014), Saffe et al. (2015), and Delgado Mena et al. (2017). For Sc, Ti, and $\mathrm{Cr}$ our final list includes both neutral and singly-ionized lines, whilst for Y, Ba, and Ce only singly-ionized lines are available. For the rest of the elements, only neutral lines were used. Hyperfine splitting (HFS) was taken into account for V, Mn, Co, $\mathrm{Cu}$, and $\mathrm{Ba}$ using the blends driver and the HFS constants of Kurucz \& Bell (1995).

We applied non-local thermodynamic equilibrium (NLTE) corrections to the oxygen abundances, obtained from the $\lambda 7771$ $5 \AA$ infrared triplet, using the grid of Ramírez et al. (2007). We also obtained NLTE abundances for $\mathrm{Na}$, using the corrections interpolated from the tables of Lind et al. (2009) through the INSPECT database ${ }^{22}$.

The abundances of $\mathrm{C}, \mathrm{N}, \mathrm{Li}, \mathrm{S}$, and also the carbon ${ }^{12} \mathrm{C} /{ }^{13} \mathrm{C}$ isotopic ratio were derived by fitting synthetic spectra to the data using the synth driver of the MOOG code. Carbon abundances

\footnotetext{
22 wWW. inspect-stars.com
} 


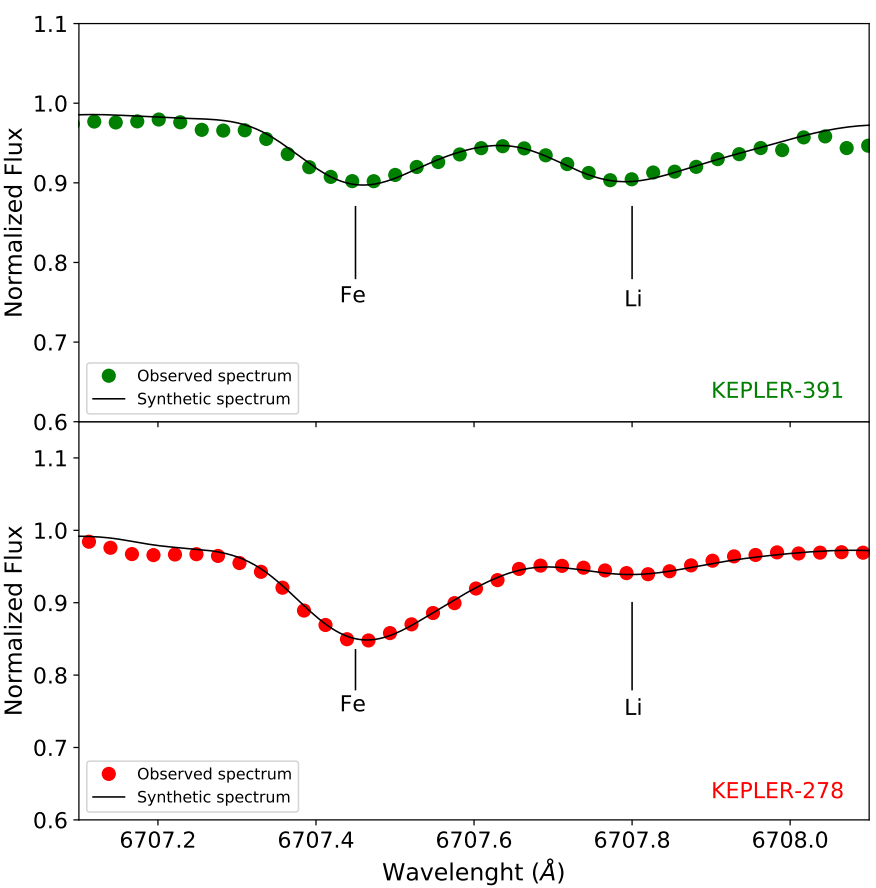

Fig. 6. Best fit obtained between the synthetic and the observed GRACES spectra of Kepler-278 and Kepler-391 around the Li I $\lambda 6708$ feature.

were derived from the $\mathrm{C}_{2}$ Swan band at $\lambda 5086$ and $\lambda 5135$ using a line list from the VALD line database (Kupka et al. 1999). Abundances of $\mathrm{N}$ and the ${ }^{12} \mathrm{C} /{ }^{13} \mathrm{C}$ isotopic ratio were computed by fitting ${ }^{12} \mathrm{CN}$ and ${ }^{13} \mathrm{C}$ features in the range 8002-8004 $\AA$ using the line list of Carlberg et al. (2012). We employed the following molecular dissociation energies: $D_{0}=6.21(\mathrm{C} 2$; Huber \& Herzberg 1979), and $D_{0}=7.65$ (CN; Bauschlicher et al. 1988). For lithium, we analyzed the Li I feature at $\lambda 6707.8 \AA$ adopting the line list of Carlberg et al. (2012), which includes blends from atomic and molecular (CN) lines. We obtained NLTE lithium abundances using the corrections by Lind et al. (2009) via the INSPECT database. In the case of sulphur, we fitted the S I features at $\lambda 6757.15 \AA$, and $\lambda 8694.62 \AA$ using the atomic data of Takeda et al. (2016). Figure 6 shows the best fits to the Li features for both stars.

The final computed abundances, relative to the solar values from Asplund et al. (2009), together with the errors are listed in Table B.1. Here, $\sigma_{\text {lines }}$ corresponds to the line-by-line abundance dispersion for each element whilst $\sigma_{\text {pars }}$ indicates the error introduced by propagating our formal uncertainties in the atmospheric parameters (see, e.g., Ramírez \& Allende Prieto 2011; Morel et al. 2014). As usual, the formal total error given in the last column of Table B.1 is obtained by adding quadratically all the error contributions.

\subsubsection{Comparison with literature}

In addition to the atmospheric parameters, BF18 also derived chemical abundances for 15 elements $(\mathrm{C}, \mathrm{N}, \mathrm{O}, \mathrm{Na}, \mathrm{Mg}, \mathrm{Al}$, $\mathrm{Si}, \mathrm{Ca}, \mathrm{Ti}, \mathrm{V}, \mathrm{Cr}, \mathrm{Mn}, \mathrm{Fe}, \mathrm{Ni}$, and $\mathrm{Y}$ ) via spectral synthesis of low S/N HIRES spectra through the SME code (Valenti \& Piskunov 1996). In Fig. 7 we show the abundances of Kepler278 and Kepler-391 derived in this work in comparison with those obtained by BF18. In general, there is a good agreement between both measurements for most elements in common. Our
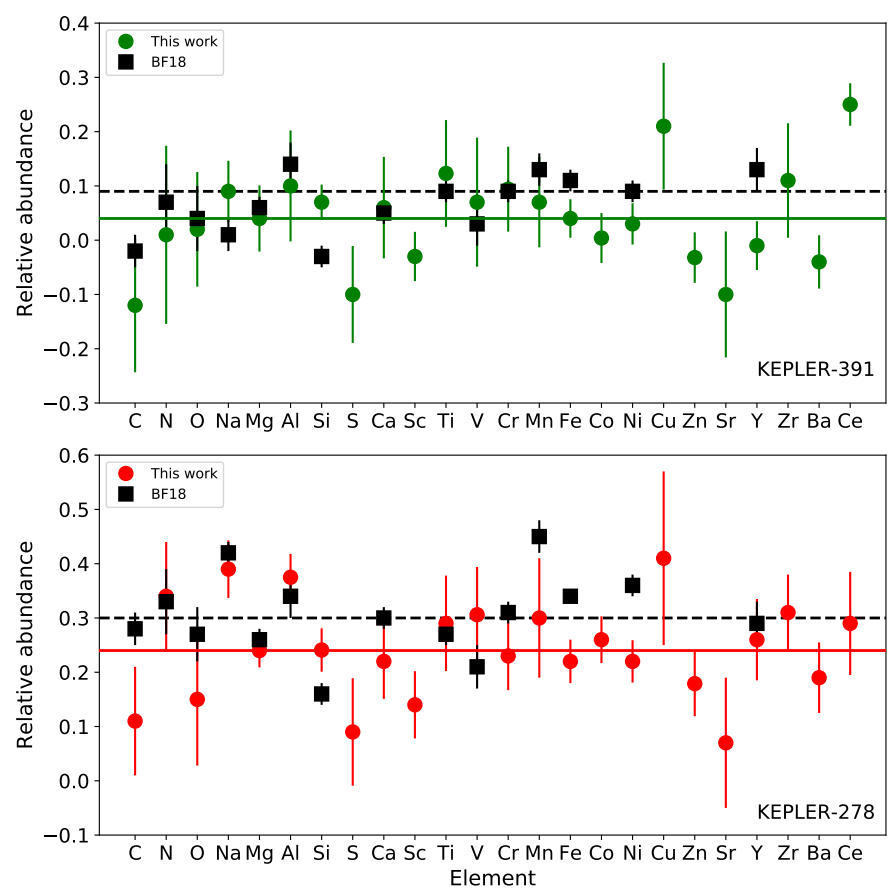

Fig. 7. Elemental abundances derived in this work (filled circles) in comparison to those reported by BF18 (filled squares) for Kepler-278 (bottom) and Kepler-391 (top). Continuous and dashed lines indicates the mean metallicities obtained in this work and in BF18, respectively.

mean metallicities (continuous lines) are slightly lower than the ones of BF18 (dashed lines) by $-0.05 \pm 0.08$ dex for Kepler-278 and $-0.02 \pm 0.07$ dex for Kepler-391. For some elements such as $\mathrm{C}, \mathrm{Mn}, \mathrm{Fe}$, and $\mathrm{Ni}$, however, the abundances obtained by BF18 are systematically larger than our values, and the differences are even larger for Kepler-278 for which the agreement is only within $2 \sigma$. On the other hand, the abundances of Si determined by BF18 are lower than our estimations. Also, for Kepler-391, their abundance of $\mathrm{Y}$ is $\sim 0.15$ dex $(>1 \sigma)$ larger than our estimation. The systematic differences are likely related to the use of different techniques to compute abundances, atmospheric parameters (see Sect. 3.1.3), models of stellar atmospheres, and line-lists (Hinkel et al. 2016; Jofré et al. 2017). Moreover, the large difference in the $\mathrm{S} / \mathrm{N}$ between their HIRES and our GRACES spectra $(\Delta S / N \gtrsim 300$ for Kepler-278 and $\Delta S / N \gtrsim 240$ for Kepler-391) likely produces a non-negligible effect in the computed chemical abundances. An additional reason for the discrepancies could be the existence of a possible systematic flaw in the abundance analysis that, according to BF18, might be affecting their results for the evolved stars.

Another feature visible in Fig. 7 is that, similar to the uncertainties in the atmospheric parameters, error bars in the chemical abundances provided by BF18 are significantly smaller (up to $\sim 80 \%$ ) than those computed in this work. The origin of such differences is that our formal quoted errors, as explained in the previous section, include the uncertainties in the atmospheric parameters (considering both internal and external errors) and the dispersion in the line-by-line measurements, whilst those reported by BF18 correspond to statistical uncertainties from fitting models to observations only, and likely underestimate the true uncertainties.

Recently, Berger et al. (2018) measured Li abundances from EWs for 1305 Kepler targets, also based on HIRES spectra taken by the CKS. They obtain $A(\mathrm{Li})_{\mathrm{LTE}}=0.52 \pm 0.16 \mathrm{dex}$ 


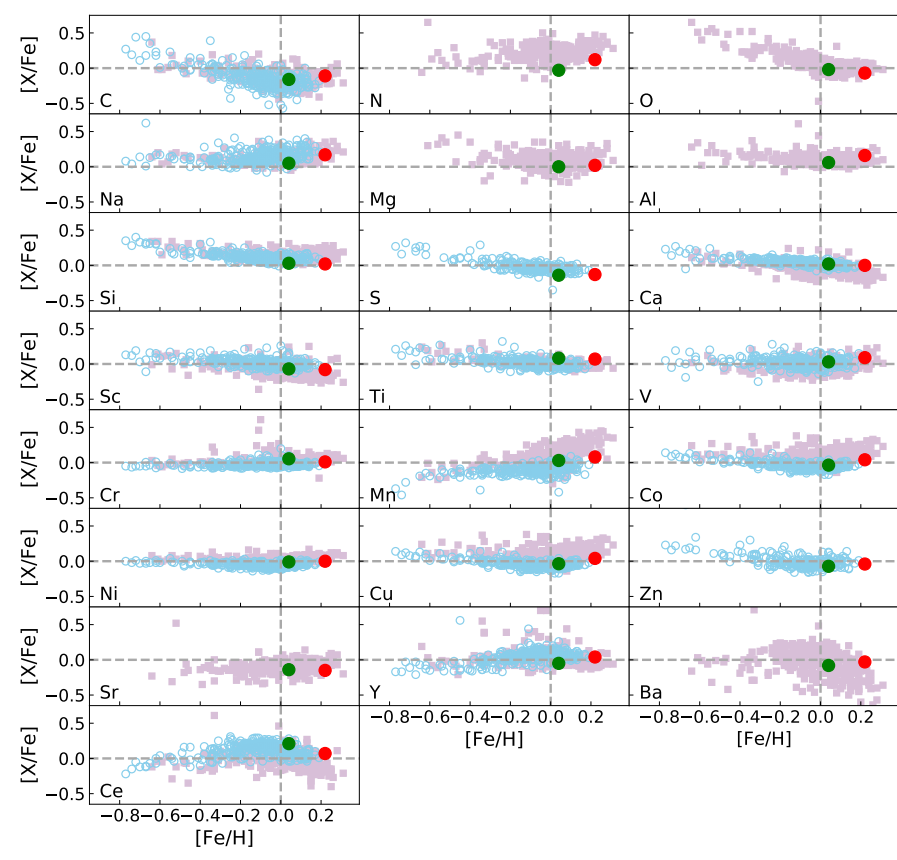

Fig. 8. Elemental abundance ratios of Kepler-278 (red circle) and Kepler-391 (green circle) compared to the Galactic chemical evolution trends by Luck \& Heiter (2007, squares) and Takeda et al. (2008b, 2016, empty circles). Dashed lines indicate the solar values.

and $A(\mathrm{Li})_{\mathrm{LTE}}=0.96 \pm 0.27$ dex for Kepler-278 and Kepler-391, respectively. These values are $0.17 \mathrm{dex}$ and $0.18 \mathrm{dex}$ smaller than our estimations for Kepler-278 and Kepler-391, respectively. As before, the discrepancies, are probably related to the use of different techniques employed (EWs vs. spectral synthesis), the difference in the adopted $T_{\text {eff }}$ (which they compiled from PE17, see Sect. 3.1.3), and the use of different data quality.

\subsubsection{Location on the $[\mathrm{X} / \mathrm{Fe}]-[\mathrm{Fe} / \mathrm{H}]$ plane and kinematic membership}

To check unusual chemical compositions, we investigated abundance trends with metallicity for all the elements. In Fig. 8, we show the $[\mathrm{X} / \mathrm{Fe}]$ abundances as a function of the $[\mathrm{Fe} / \mathrm{H}]$ of Kepler-278 and Kepler-391 compared to Galactic chemical evolution trends of solar neighborhood evolved stars. The comparison data were taken from Luck \& Heiter (2007, purple squares) for all elements except Zn, and from Takeda et al. (2008b, 2016, light blue circles) for all elements with the exception of $\mathrm{N}, \mathrm{Mg}$, $\mathrm{O}$, and $\mathrm{Ba}$. In both samples, abundances have been computed from high-resolution spectra using similar techniques to ours and are dominated by stars of the thin disk. In general, the chemical composition of both Kepler stars does not appear to be anomalous but rather consistent with the abundances of the thin-disk nearby evolved stars with similar metallicities. In particular, no important $\alpha$-element enhancement is observed neither for Kepler-278 nor Kepler-391. Averaging the abundances of O, $\mathrm{Mg}, \mathrm{Si}, \mathrm{Ca}$, and $\mathrm{Ti}$ we obtain $[\alpha / \mathrm{Fe}]=0.04$ dex for both stars The $\mathrm{Ca}$ abundance for Kepler-278 and $\mathrm{Ti}$ abundances for both stars appear slightly overabundant compared to the mean trend of both reference samples. However, they are still consistent with the abundances of the comparison stars considering the $1 \sigma$ scatter. Also, we do not observe any evident signs of anomalies in the abundance of Fe-peak or $s$-process elements.
Using proper motions and parallaxes ${ }^{23}$ from Gaia DR2 (Gaia Collaboration 2018), we derived Galactic space-velocity $U V W$ components for both stars following the procedure detailed in Jofré et al. (2015b). We find $(U, V, W)=(24.56 \pm 0.60,-32.24 \pm$ $0.19,1.26 \pm 0.15) \mathrm{km} \mathrm{s}^{-1}$ for Kepler-278 and $(U, V, W)=(36.33 \pm$ $2.33,40.43 \pm 0.71,-14.67 \pm 1.31) \mathrm{km} \mathrm{s}^{-1}$ for Kepler-391. Then, from these space-velocity components and using the membership formulation by Reddy et al. (2006), we found that the probability of belonging to the thin disk population is $\sim 98 \%$ for both stars, which is consistent with the results from the chemical analysis.

\subsubsection{Lithium abundance}

The lithium feature at $\lambda 6707.8$ is detectable in the spectra of Kepler-278 and it is even stronger on the spectra of Kepler391 as can be noticed in Fig. 6. For Kepler-278, we determined $A(\mathrm{Li})_{\mathrm{NLTE}}=0.87 \pm 0.10 \mathrm{dex}$ and $A(\mathrm{Li})_{\mathrm{NLTE}}=1.29 \pm 0.09 \mathrm{dex}$ for Kepler-391. The Li abundance of Kepler-391 is just below the standard limit, $A(\mathrm{Li}) \approx 1.5 \mathrm{dex}$, from which evolved stars are considered to have an anomalous abundance of $\mathrm{Li}$ and termed as Li-rich stars (e.g., Kumar et al. 2011). According to their $T_{\text {eff }}$ and $\log g$ values, Kepler-391, is near the base of the RGB (see Fig. 12) and then only recently started the first dredge-up (FDU). Therefore, the relatively high Li abundance of Kepler-391 is most likely a remnant from the main-sequence phase as in other similar cases (e.g., Adamów et al. 2014, 2015). In agreement with this scenario, we do not find evidence of increased stellar rotation or other chemical anomalies (see next section) that could indicate planet engulfment events (Siess \& Livio 1999; Carlberg et al. 2012; Adamów et al. 2014; Jofré et al. 2015a).

Another possibility to explain high lithium content in evolved stars is a fresh lithium production phase via the Cameron-Fowler mechanism (Cameron \& Fowler 1971). This scenario is generally associated to stars near the luminosity bump (LB, see, e.g., Fig. 2 of Jofré et al. 2015a) on the RGB, where most of lowmass lithium-enhanced evolved stars tend to cluster. However, from its position on the HR-diagram (see Fig. 12), Kepler-391 it is relatively far away from the LB position.

\subsection{5. $[\mathrm{X} / \mathrm{Fe}]$ versus $T_{\mathrm{c}}$}

Beyond the overall metallicity enhancement of main-sequence stars with planets (e.g., Ghezzi et al. 2010b), it has been suggested that trends in elemental abundances with condensation temperature $\left(T_{\mathrm{c}}\right)$ are possible signatures of planet formation and evolution processes. A deficit of refractory elements $\left(T_{\mathrm{c}} \gtrsim\right.$ $900 \mathrm{~K} ; \alpha$-group and iron-peak elements) relative to volatiles $\left(T_{\mathrm{c}}<900 \mathrm{~K}\right.$, e.g., C, O, S) on the stellar atmosphere might indicate this material was sequestered in the formation of planetesimals, rocky planets, or the cores of giant planets (Meléndez et al. 2009; Ramírez et al. 2010). On the other hand, an overabundance of refractory elements in comparison to volatiles might indicate the accretion of hydrogen-depleted rocky material onto the star (Gonzalez 1997; Murray \& Chaboyer 2002; Meléndez et al. 2017; Saffe et al. 2017).

Recently, Maldonado \& Villaver (2016, M16 hereafter) analyzed the abundances of different groups of stars (dwarfs, subgiants, and giants), with and without planets, as a function of $T_{\mathrm{c}}$ in order to search for differences that could be linked to

23 We corrected the Gaia parallaxes for the $82 \mu$ arcsec suggested by Stassun \& Torres (2018) and derived the distances from these values (see Table 1). 

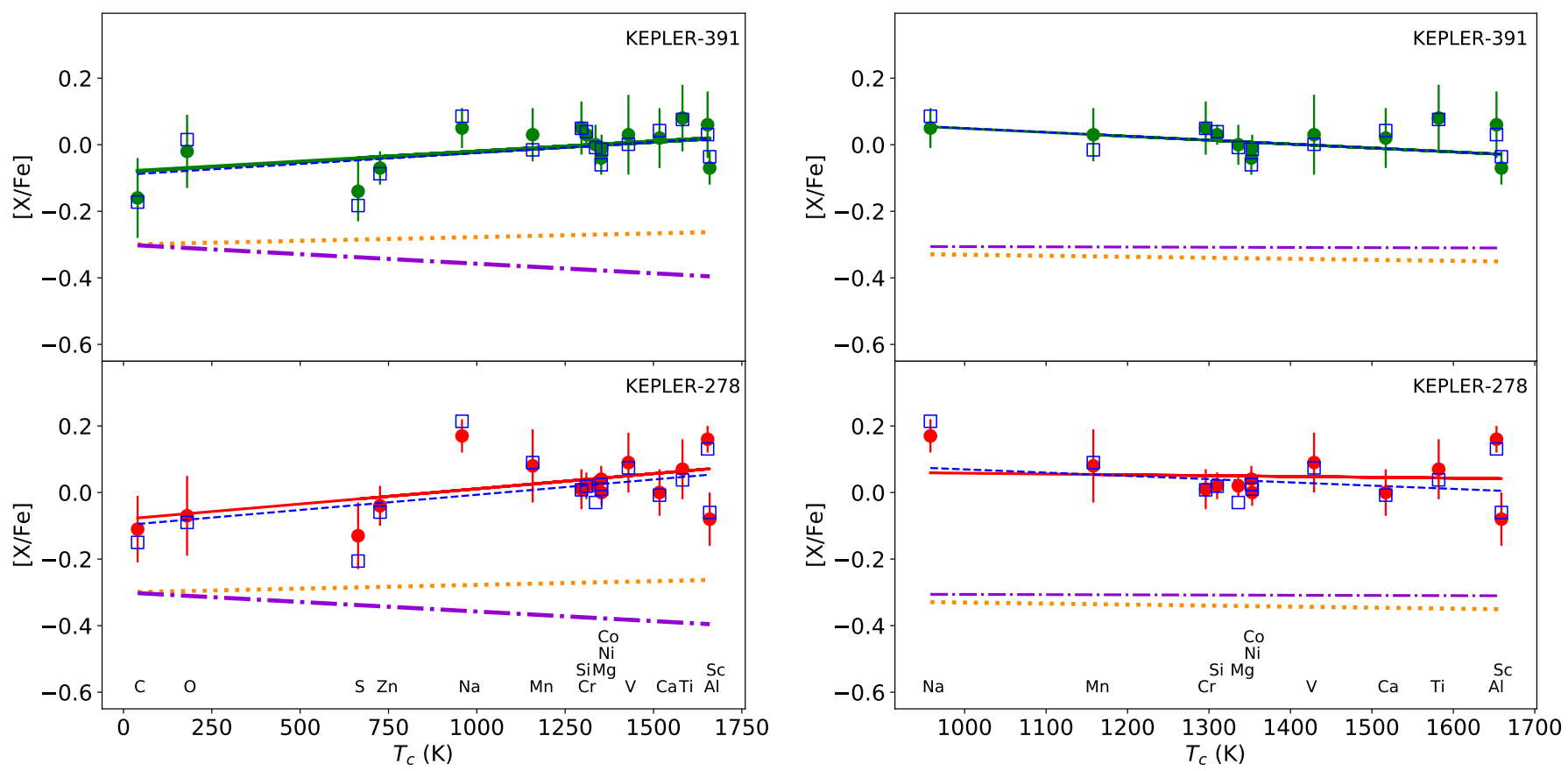

Fig. 9. Left: abundances of volatile and refractory elements for Kepler-278 (bottom) and Kepler-391 (top) as a function of dust condensation temperature; filled circles and blue squares represent $[\mathrm{X} / \mathrm{Fe}]$ without and with Galactic chemical evolution (GCE) corrections, respectively. Solid and dashed lines show the weighted linear fits to the abundance values without and with GCE corrections, respectively. Orange dotted and purple dot-dashed lines show the mean trend found by M16 for subgiants and giants with planets, respectively. Right: same as left panel, but for refractory elements only.

the planet formation process. Considering all elements (volatiles and refractories), these authors found no significant difference between the slopes of evolved stars with and without planets. When restricting the analysis to refractory elements, however, they found differences between stars with and without known planets for the samples of main-sequence and subgiant stars but no for the sample of giants. Given that the sample of main-sequence and subgiant stars contain less massive and older stars than the sample of giants, M16 consider that Galactic radial mixing offers a more suitable scenario for explaining the observed trends in main-sequence and subgiant stars rather than planet formation.

In the left panel of Fig. 9 we show our $[\mathrm{X} / \mathrm{Fe}]$ values as function of $T_{\mathrm{c}}$ for Kepler-278 and Kepler-391, considering both volatile and refractory elements in common with those analyzed by M16 (16 elements). For $T_{\mathrm{c}}$, we have used the $50 \%$ values from Lodders (2003). No significant trend is observed for Kepler-278 nor for Kepler-391. A weighted linear fit reveals small positive slopes of $(9.12 \pm 6.03) \times 10^{-5}$ dex $\mathrm{K}^{-1}$ for Kepler-278 and $(6.02 \pm 4.14) \times 10^{-5}$ dex K ${ }^{-1}$ with standard fit deviations of 0.07 and 0.05 dex for Kepler-278 and Kepler-391, respectively. In both cases the slopes are consistent, within the errors, with the average value presented by M16 for the subgiants with planets sample: $(2.24 \pm 1.17) \times 10^{-5} \mathrm{dex} \mathrm{K}^{-1}$, rather than the one obtained for giants with planets sample: $(-5.76 \pm 1.58) \times 10^{-5} \mathrm{dex} \mathrm{K}^{-1}$.

We also applied the Galactic chemical evolution (GCE) corrections to our $[\mathrm{X} / \mathrm{Fe}]$ values based on the studies of González Hernández et al. (2013) following the same procedure as Saffe et al. $(2015,2017)$. These corrections are very small for both stars and therefore there are no significant changes in the slopes values. In the right panel of Fig. 9 we show the $[\mathrm{X} / \mathrm{Fe}]$ vs. $T_{\mathrm{c}}$ trends when only the refractory elements common to the study of M16 are considered (12 elements). As before, no evident trend in the abundances as a function of $T_{\mathrm{c}}$ is present for any of the stars. Weighted linear fits to the data result in negative slopes for both Kepler-278 $\left(-2.45 \pm 8.91 \times 10^{-5}\right.$ dex K $\left.{ }^{-1}\right)$ and Kepler-391 $\left(-11.8 \pm 7.08 \times 10^{-5} \mathrm{dex} \mathrm{K}^{-1}\right)$ in agreement with the negative average slopes found by M16 for subgiants with planets $(-3.06 \pm$ $2.32 \times 10^{-5}$ dex $\left.\mathrm{K}^{-1}\right)$ and giants with planets $(-0.62 \pm 2.35 \times$ $10^{-5}$ dex $\mathrm{K}^{-1}$ ). Again, similar negative slopes are observed when GCE corrections are considered.

\section{Planetary properties}

\subsection{Transit photodynamical modeling}

We analyzed the Kepler data using a photodynamical model (Carter et al. 2011) described in Almenara et al. (2018a). Briefly, we used the REBOUND code (Rein \& Liu 2012), with the WHFast $N$-body integrator (Rein \& Tamayo 2015), to compute the positions of the star and the planets during the Kepler observations. The latter is used to compute the light curve model with the analytic description of Mandel \& Agol (2002) using a quadratic limb-darkening law (Manduca et al. 1977) and the parameterization of Kipping (2013) to consider only physical values. To model the long-cadence data, we oversampled the model by a factor of 10 and then binned back to the observed cadence. This accounts for the deformation of the signal due to the duration of the exposure (Kipping 2010). With an $N$-body time-step of $0.05 \mathrm{~d}$ we estimate the error of the model to be lower than $1 \mathrm{ppm}$ following Almenara et al. (2018a).

For Kepler-278, the model has 22 free parameters: the stellar density, two limb-darkening coefficients, five orbital elements, and a mass and radius ratio per planet, the difference in longitudes of the ascending nodes, the amplitude of an additional multiplicative white noise term for the Kepler long and short-cadence data, and a free normalization factor for each dataset, corresponding to the out-of-transit flux. The five orbital 
parameters of each planet and the difference in longitudes of the ascending node are set at the reference time 2455695.37632 BJD $_{\text {TDB }}$ for Kepler-278, and 2455690.39125 $\mathrm{BJD}_{\mathrm{TDB}}$ for Kepler-391. For Kepler-391 there is only longcadence data so the model has 20 parameters.

We used a normal prior for the stellar density $\left(\rho_{\star}=\right.$ $0.07240 \pm 0.00094 \mathrm{~g} \mathrm{~cm}^{-3}$ for Kepler-278 from H13, and $\rho_{\star}=$ $0.077 \pm 0.011 \mathrm{~g} \mathrm{~cm}^{-3}$ for Kepler-391, Sect. 3.3), and uniform prior distributions for the remaining parameters. We limit the inclination of the inner planet to the range $[0,180]$ degrees and the inclination of the outer planet to the range $[0,90]$ degrees due to the symmetry of the problem.

We used the emcee algorithm (Goodman \& Weare 2010; Foreman-Mackey et al. 2013) to sample from the posterior distributions of the parameter models. We ran 100 walkers for $1.2 \times 10^{6}$ steps for Kepler-278, and $0.7 \times 10^{6}$ steps for Kepler391. Only the last 100000 steps were used for the final inference.

\subsection{Results}

The photodynamical modeling allows improving the precision of the planetary parameters significantly with respect to an analysis correcting the light curves using individually-measured transit times. This can be particularly important in low $\mathrm{S} / \mathrm{N}$ regimes, such as is the case for Kepler-278 and Kepler-391, where the transit times measured on individual transits are plagued by biases from spot crossing and other stellar variability issues (see, e.g., Barros et al. 2013). One of the largest advantages of the photodynamical modeling is minimizing the impact of these effects by using the entire set of transits to constrain each transit time and analyzing the dynamics of the system (Almenara et al. 2015). However, even with a photodynamical analysis, the small S/N of the transits of Kepler-278 and Kepler-391 calls for caution. For example, the mean stellar density can be inferred with a precision of around $50 \%$ from the transit light curve analysis for Kepler-278, but the asteroseismic analysis provides a precision of $1.3 \%$ (H13). The inference may be biased by unmodelled or unknown systematics effects, that at this level of signal dominate the error budget. The two-dimensional projections of the posterior sample are shown in Figs. B.6 and B.7 whilst the summary statistics of the marginal posterior distributions of each parameter is presented in Table 2. For each value, we report the median and $68.3 \%$ confidence interval. For certain parameters, such as the eccentricities and mass ratios of the planets around Kepler-391, only upper limits are available. In this case, we report only the upper limit of the $95 \%$ Highest Density Interval $(\mathrm{HDI})^{24}$.

Concerning the planets around Kepler-278, the inference based on the photodynamical model indicates that the planets are in eccentric $(e \sim 0.7)$ aligned (similar arguments of the pericentre, inclinations and longitudes of the ascending nodes) orbits. The posterior of the mean stellar density is dominated by the asteroseismic prior. The planet/star radius ratio values, $R_{\mathrm{p}} / R_{*}$, are known with precisions of around $2.3 \%$, and combined with the stellar radius computed in Sect. 3.3, they yield planetary radii of $3.96 \pm 0.08 R_{\oplus}$, and $3.27 \pm 0.07 R_{\oplus}$, for planets b and c, respectively. Because the planets exhibit transit timing variations (Fig. 10, left; see also Sect. 5.2), the mass ratios $M_{\mathrm{p}} / M_{*}$ can also be determined, albeit with a precision of around $40 \%$ for both

\footnotetext{
${ }^{24}$ The $q-\%$ Highest Density Interval on a one-dimensional density is defined by all the points such that their densities are larger than a given value $W$ and such that the integral over all those values is $q / 100$, i.e., the inteval $I=\{x: f(x)>W\}$ where $W$ satisfies that $\int_{x: f(x)>W} f(x)=q / 100$.
}

planets. The posterior distributions of the mass ratios exhibit two or three not fully separated modes, indicating that the solution is not unique, and highlighting the difficulty of exploring the parameter space of the photodynamical model when transits have low S/N (see also Almenara et al. 2018a). The 95\% upper limits for the masses are $127.12 M_{\oplus}\left(0.4 M_{\mathrm{Jup}}\right)$, and $54 M_{\oplus}\left(0.17 M_{\mathrm{Jup}}\right)$, for planets $\mathrm{b}$ and $\mathrm{c}$, respectively. The planets are, therefore, likely to be Neptune-like, but their density is very poorly constrained.

The orbits of the planets around Kepler-391 are compatible with circular orbits, according to the inference using the photodynamical model, but their eccentricities are badly constrained, with $95 \%$ upper limits of 0.46 and 0.27 for the inner and outer planet, respectively. The difference of the longitudes of the ascending nodes is compatible with zero, but the value is again, poorly constrained. In this case, the stellar density changes slightly from the prior, but the precision is not much improved by the inclusion of the data. Because no TTV are observed (Fig. 10, right) only upper limits on the masses can be measured. Assuming the stellar mass from Sect. 3.3, we find that the upper limits of the $95 \%$-HDI are $4.4 M_{\text {Jup }}$ and $4.8 M_{\text {Jup }}$, for planets $\mathrm{b}$ and $\mathrm{c}$, respectively. In fact, the posterior distribution of planet $\mathrm{b}$ is slightly bimodal, and the $95 \%$ HDI is disjointed: $[0.0,2.3] \cup[3.2,4.4] M_{\text {Jup }}$. In any case, the data do not provide strong constraints on the planetary masses. On the other hand, the radii are determined with a precision of around $10 \%$, and indicate the planets are sub-Neptunes.

Finally, we combined the stellar parameters derived earlier in Sect. 3.3 with the relative parameters from the photodynamical analysis to compute other physical properties for all the planets. Our final planet properties are listed in Table 2.

In Fig. 11 we show the comparison between our planetary radii and those from B11, BA13, H13, R14, Rowe et al. (2015, R15, hereafter), J17, FP18, and BE18. In general, as can be noticed, our radii agree fairly well with those obtained previously, particularly for Kepler-391b/c. Alternatively, for Kepler$278 \mathrm{~b} / \mathrm{c}$ although most of the results are in relatively good agreement (within $1 \sigma$ ), we note that a few estimations from literature (e.g., B11, BA13, H13) agree with our values only within $2 \sigma$. The discrepancies with B11 and BA13 mainly arise owing to their stellar radii are $\sim 37 \%$ larger and $\sim 36 \%$ smaller, respectively, than our estimation (see Fig. 5). Additionally, although within $1 \sigma$, the large discrepancies with the estimations of J17 are also originated from the differences in the stellar radius. The disagreement with the planetary radii derived by $\mathrm{H} 13$ can be explained from significant differences between our derived radius ratio values $R_{\mathrm{p}} / R_{\star}$ and those computed by BA13 that are adopted by $\mathrm{H} 13$. The radius ratio values determined by BA13 are 16 and $6 \%$ larger than ours for Kepler-278b and Kepler-278c, respectively. The disagreement is likely related to different methods employed to analyze the Kepler light curves (i.e., photodynamical vs. individual transit curves; Almenara et al. 2018a), which also includes significant differences in the assumption for excentricity, limb-darkening, and normalization, among others. A similar scenario possibly explains the small discrepancy with the radius of Kepler-278b derived by R15.

Our estimations of semi-major axis and incident flux are in good agreement with the available results computed by $\mathrm{H} 13$, R14, R15, J17, and FP18. Finally, the masses of the planets around Kepler-278 presented here are independent of the measured stellar mass given that they are computed from the planetary radii and densities derived from the photodynamical model using the asteroseismic stellar density. 
Table 2. Planetary parameters of the Kepler-278 and Kepler-391 systems.

\begin{tabular}{|c|c|c|c|c|}
\hline Parameter & Kepler-278b & Kepler-278c & Kepler-391b & Kepler-391c \\
\hline \multicolumn{5}{|c|}{ Model parameters } \\
\hline $\begin{array}{l}\text { Kepler long-cadence additional } \\
\text { white noise level }\end{array}$ & \multicolumn{2}{|c|}{$2.495 \pm 0.040$} & \multicolumn{2}{|c|}{$1.3993 \pm 0.0095$} \\
\hline $\begin{array}{l}\text { Kepler } \text { short-cadence additional } \\
\text { white noise level }\end{array}$ & \multicolumn{2}{|c|}{$1.1317 \pm 0.0032$} & \multicolumn{2}{|c|}{ N/A } \\
\hline Stellar mean density, $\rho_{\star}\left[\mathrm{g} \mathrm{cm}^{-3}\right]^{(a)}$ & \multicolumn{2}{|c|}{$0.07237 \pm 0.00096$} & \multicolumn{2}{|c|}{$0.0822_{-0.012}^{+0.0099}$} \\
\hline Linear limb darkening, $u_{\mathrm{a}}$ & \multicolumn{2}{|c|}{$0.74_{-0.16}^{+0.13}$} & \multicolumn{2}{|c|}{$0.30_{-0.21}^{+0.32}$} \\
\hline Quadratic limb darkening, $u_{\mathrm{b}}$ & \multicolumn{2}{|c|}{$-0.27_{-0.12}^{+0.22}$} & \multicolumn{2}{|c|}{$0.31_{-0.40}^{+0.31}$} \\
\hline Scaled semi-major axis, $a / R_{\star}$ & $15.152 \pm 0.067$ & $21.541 \pm 0.096$ & $6.20_{-0.31}^{+0.24}$ & $12.23_{-0.62}^{+0.47}$ \\
\hline Eccentricity, $e^{(b)}$ & $0.696_{-0.026}^{+0.017}$ & $0.616_{-0.023}^{+0.015}$ & $<0.46^{(c)}$ & $<0.27$ \\
\hline Inclination, $i\left[^{\circ}\right]^{(d)}$ & $85.11_{-0.52}^{+0.72}$ & $88.58_{-0.51}^{+1.2}$ & $85.5_{-1.7}^{+2.9}$ & $87.27_{-0.48}^{+0.72}$ \\
\hline Argument of pericentre, $\omega\left[^{\circ}\right]$ & $21.1 \pm 7.7$ & $20.80 \pm 7.9$ & $103_{-46}^{+53}$ & $126_{-63}^{+100}$ \\
\hline $\begin{array}{l}\text { Difference of the longitude of the } \\
\text { ascending node, } \Omega_{1}-\Omega_{2}\left[{ }^{\circ}\right]\end{array}$ & \multicolumn{2}{|c|}{$10_{-32}^{+10}$} & \multicolumn{2}{|c|}{$0 \pm 74$} \\
\hline$\sqrt{e} \cos \omega$ & $0.778_{-0.056}^{+0.039}$ & $0.735_{-0.055}^{+0.037}$ & $-0.06 \pm 0.32$ & $-0.077_{-0.22}^{+0.25}$ \\
\hline$\sqrt{e} \sin \omega$ & $0.295 \pm 0.098$ & $0.274 \pm 0.093$ & $0.35_{-0.21}^{+0.14}$ & $0.16 \pm 0.22$ \\
\hline Mean anomaly, $M_{0}$ & $11.2_{-1.1}^{+1.4}$ & $340.7 \pm 1.7$ & $321+28$ & $227 \pm 63$ \\
\hline Impact parameter, $b$ & $0.544_{-0.085}^{+0.066}$ & $0.27_{-0.23}^{+0.11}$ & $0.38_{-0.26}^{+0.23}$ & $0.561_{-0.019}^{+0.087}$ \\
\hline Epoch of transit, $T_{0}\left[\mathrm{BJD}_{\mathrm{TDB}}\right]$ & $2455695.3727_{-0.0095}^{+0.00036}$ & $2455700.4118 \pm 0.0052$ & $2455690.3959_{-0.0089}^{+0.013}$ & $2455696.9123_{-0.0074}^{+0.0085}$ \\
\hline Period, $P[\text { days }]^{(e)}$ & $30.1556_{-0.0020}^{+0.0041}$ & $51.111_{-0.011}^{+0.023}$ & $7.4175_{-0.0037}^{+0.0027}$ & $20.501_{-0.010}^{+0.032}$ \\
\hline Radius ratio, $R_{\mathrm{p}} / R_{\star}$ & $0.01266_{-0.00027}^{+0.00023}$ & $0.01046 \pm 0.00025$ & $0.00795_{-0.00027}^{+0.00034}$ & $0.00915 \pm 0.00034$ \\
\hline Mass ratio, $M_{\mathrm{p}} / M_{\star}$ & $0.000142_{-0.000033}^{+0.000095}$ & $0.000087_{-0.000050}^{+0.000026}$ & $<0.0026$ & $<0.0036$ \\
\hline Planet mean density, $\rho_{\mathrm{p}}\left[\mathrm{g} \mathrm{cm}^{-3}\right]$ & $5.0_{-1.1}^{+3.2}$ & $5.5_{-3.2}^{+1.5}$ & $<380$ & $<400$ \\
\hline \multicolumn{5}{|c|}{ Derived properties } \\
\hline Semi-major axis, $a[\mathrm{AU}]$ & $0.202 \pm 0.005$ & $0.287 \pm 0.007$ & $0.083_{-0.013}^{+0.012}$ & $0.164_{-0.022}^{+0.026}$ \\
\hline Radius, $R_{\mathrm{p}}\left[R_{\oplus}\right]$ & $3.955 \pm 0.080$ & $3.268 \pm 0.067$ & $2.50 \pm 0.29$ & $2.88 \pm 0.33$ \\
\hline Mass, $M_{\mathrm{p}}\left[M_{\oplus}\right]$ & $56+13$ & $34.9_{-21}^{+9.9}$ & $<1100$ & $<450$ \\
\hline Incident flux, $S_{\text {inc }}\left[S_{\oplus}\right]$ & $110 \pm 14$ & $54.3 \pm 7.2$ & $690+390$ & $179{ }_{-110}^{+96}$ \\
\hline Equilibrium temperature, $T_{\mathrm{eq}}[\mathrm{K}]^{(f)}$ & $586 \pm 19$ & $492 \pm 16$ & $930 \begin{array}{c}+37 \\
-140\end{array}$ & $662+89$ \\
\hline Semiamplitude, $K_{\star}\left[\mathrm{m} \mathrm{s}^{-1}\right]$ & $14.0_{-4.7}^{+21}$ & $6.7_{-5.1}^{+2.4}$ & $<340$ & $<93$ \\
\hline
\end{tabular}

Notes. ${ }^{(a)}$ For Kepler-278 we used as prior the stellar density derived from asteroseismology by H13 and the one determined from stellar models in Sect. 3.3 for Kepler-391. ${ }^{(b)}$ The orbital parameters correspond to Jacobi elements computed at the reference time $t_{\text {ref }}=2455695.37632$ BJD for Kepler-278 and at $t_{\mathrm{ref}}=2455696.9123 \mathrm{BJD}_{\mathrm{TBD}}$ for Kepler-391. ${ }^{(c)}$ All upper limits correspond to the $95 \%$ confidence interval. ${ }^{(d)}$ The prior for the inclination of planets $\mathrm{b}$ is uniform between $[0,180]^{\circ}$, and $[0,90]^{\circ}$ for planets $\mathrm{c}$. The value for the inclination in the table for planets $\mathrm{b}$ is reflected with respect to $i=90^{\circ}$, the supplementary angle is equally probable. ${ }^{\left({ }^{e}\right)}$ Defined as $P \equiv \sqrt{\frac{3 \pi}{G \rho_{\star}}\left(\frac{a}{R_{\star}}\right)^{3}}$. ${ }^{(f)}$ Calculated with Eq. (4) from Johnson et al. (2017) assuming a Bond albedo $\alpha$ of 0.3 .

\section{Discussion}

\subsection{Host stars ascending the red giant branch}

Figure 12 shows the location of Kepler-278 and Kepler-391 in the $T_{\text {eff }}-\log g$ plane in comparison with other confirmed exoplanet hosts detected via RVs and transits. Both stars, with similar early K spectral types, lie close to the base of the RGB at the boundary between the luminosity classes of giants and subgiants. Their derived surface gravities are more compatible with their classification as subgiants $(3.5<\log g<4.1$, Bastien et al. 2016). Based on the criteria to distinguish between subgiants and giants, that relies on the bolometric magnitude $M_{\mathrm{bol}}$, both Kepler-278 $\left(M_{\text {bol }}=3.12\right)$ and Kepler-391 $\left(M_{\text {bol }}=3.36\right)$ would be also classified as subgiants ${ }^{25}$. However, the two stars are classified as giants according to physically motivated boundaries from solar metallicity interior models (Huber et al. 2017; Berger et al. 2018 ), using the evolstate code ${ }^{26}$. In any case, consistent with their location on the HR-diagram, we obtained a relatively high carbon isotopic ratio, ${ }^{12} \mathrm{C} /{ }^{13} \mathrm{C}>40$ (no detection) for both stars, indicating that $\mathrm{CN}$-cycled material is little or not yet well mixed (e.g., Gilroy \& Brown 1991; Thorén et al. 2004; Afşar et al. 2012) and therefore confirming that both stars are just starting their ascent on the RGB.

$25 \quad M_{\text {bol }}>2.82$ : subgiants; $M_{\text {bol }}<2.82$ : giants (Ghezzi et al. 2010a; Maldonado et al. 2013; Jofré et al. 2015b).

${ }^{26}$ See https://github.com/danxhuber/evolstate 

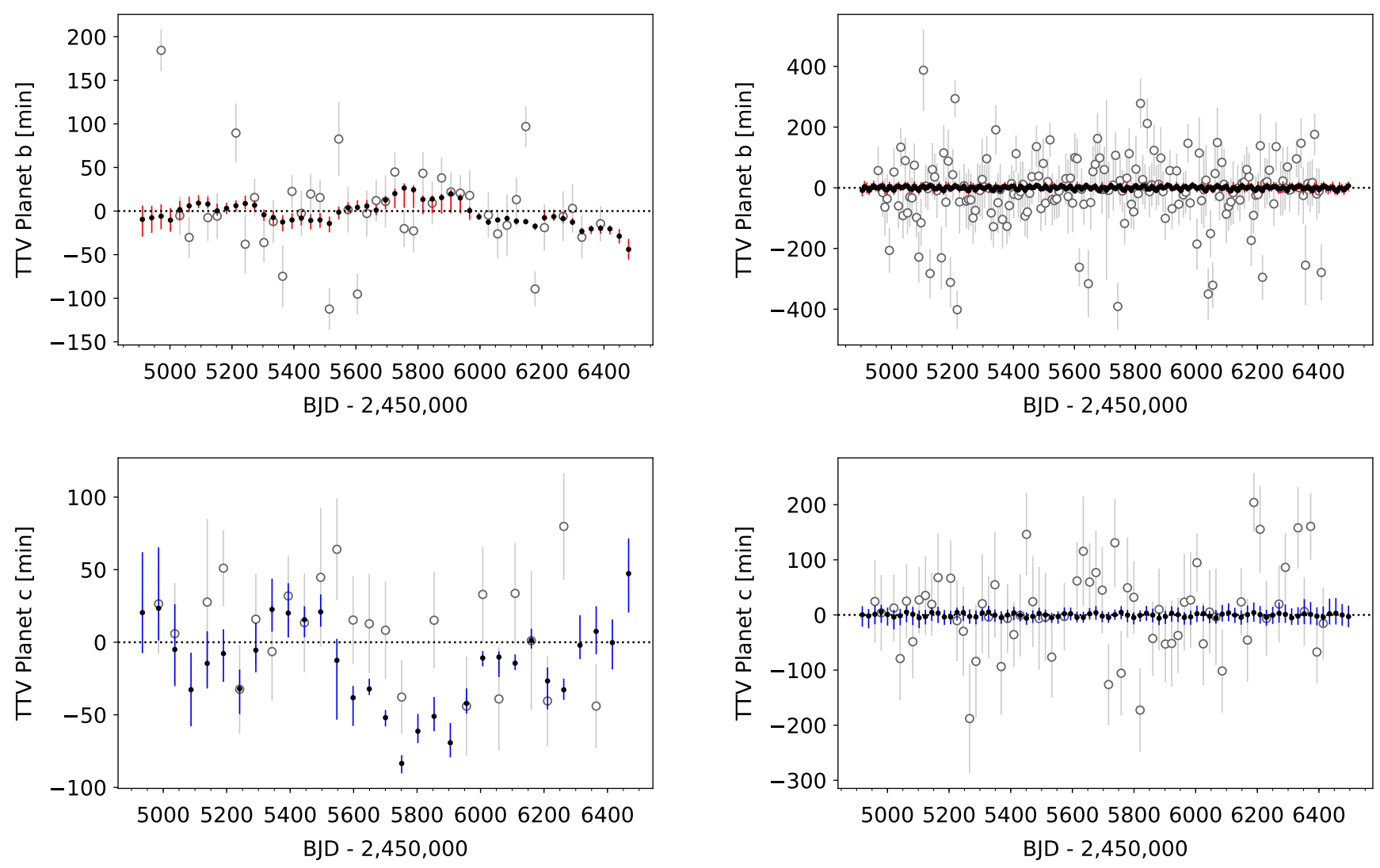

Fig. 10. Posterior TTV of Kepler-278 b (top left panel, red), Kepler-278c (bottom left panel, blue), Kepler-391 b (top right panel, red), and Kepler$391 \mathrm{c}$ (bottom right panel, blue) from the photodynamical modeling. For comparison the TTV of Rowe et al. (2015) measured on individual transits are shown as empty circles with grey errorbars.

As can be noticed in Fig. 12, there are several stars all along the RGB with planets detected from RV surveys. However, only a few late subgiants and early red giants $(\sim 15)$ are known to host transiting planets, which highlight the difficulty to detect planetary transits at this evolutionary stage. Recently, Huber et al. (2019) presented the first oscillating late subgiant star, TOI-197, with a transiting planet discoverd by TESS. The stellar parameters of TOI-197 $\left(T_{\text {eff }}=5080 \pm 90 \mathrm{~K}, \log g=3.60 \pm 0.08 \mathrm{dex}\right.$, $M_{\star}=1.21 \pm 0.07 M_{\odot}$, and $R_{\star}=2.94 \pm 0.06 R_{\odot}$ ) are very similar to those of Kepler-278 and Kepler-391. Moreover, the measured $\Delta v$ value of $28.94 \pm 0.15 \mu \mathrm{Hz}$ in TOI-197 (Huber et al. 2019), indicating that the star has just started its ascent on the RGB (Mosser et al. 2014), is also very similar to that of Kepler-278.

\subsection{TTV in the system Kepler-278}

The first sinusoidal TTV signals for Kepler-278c were reported by Van Eylen \& Albrecht (2015), and later by Holczer et al. (2016). Also, R15 measured TTV in the Kepler-278 system and included their effect in the transit models. However, none of these works reported long-term TTV signals for the inner planet Kepler-278b. The increased precision in the transit times obtained from the photodynamical analysis allowed us not only to confirm the presence of a TTV signal in the outer planet but also to show, for the first time, a TTV signal in Kepler-278b (upper left panel in Fig. 10), and hence estimate the mass of the outer planet. For comparison, in Fig. 10 we also show the TTV of R15 measured on individual transits (empty circles). As can be seen, the error bars of the transit times based on individual measurements are considerably larger than those from the photodynamical analysis (e.g., Almenara et al. 2018a), which in the case of the planets around Kepler-278 hinder the detection of a TTV signal in the inner planet.

Using the Bayesian information criterion $\left(\mathrm{BIC}^{27}\right.$, Schwarz 1978), we found that the photodynamical model with the planetary masses as free parameters (model with TTV), as derived in Sect. 4.1 for the system Kepler-278, provides a better fit to the data than a model where the planetary masses are fixed to zero (model without TTV). We obtained $\mathrm{BIC}_{\mathrm{TTV}}=-813267.0$ and $\mathrm{BIC}_{\mathrm{no}-\mathrm{TTV}}=-813226.8$ for the first and second case, respectively. Considering these values we derived $\triangle \mathrm{BIC} \sim 40$, which indicates a very strong case for TTV (Kass \& Raftery 1995).

It is also interesting to note that the posterior TTV of Kepler278b (top) and Kepler-278c (bottom) are anticorrelated. The presence of anticorrelated TTV signals among planet candidates on a single target provides strong evidence that the objects are true interacting planets (Steffen et al. 2013, and references therein). However, our posterior TTV are conditional to the model hypothesis that both planets are in the same system and interact gravitationally. Therefore, the anti-correlation of our TTV is simply a consequence of this hypothesis.

\subsection{Planets in the mass-radius diagram}

Figure 13 shows the location of Kepler-278b and Kepler-278c in a mass-radius diagram compared to other relatively small transiting planets $\left(R_{\mathrm{p}}<4.5 R_{\oplus}\right)$ with measured masses and radii

${ }^{27} \mathrm{BIC}=-2 \ln \mathcal{L}_{\max }+d \ln n$; where $\mathcal{L}_{\max }$ is the maximum value of the Likelihood function, $d$ is the number of free parameters in the model, and $n$ is the number of data-points. 

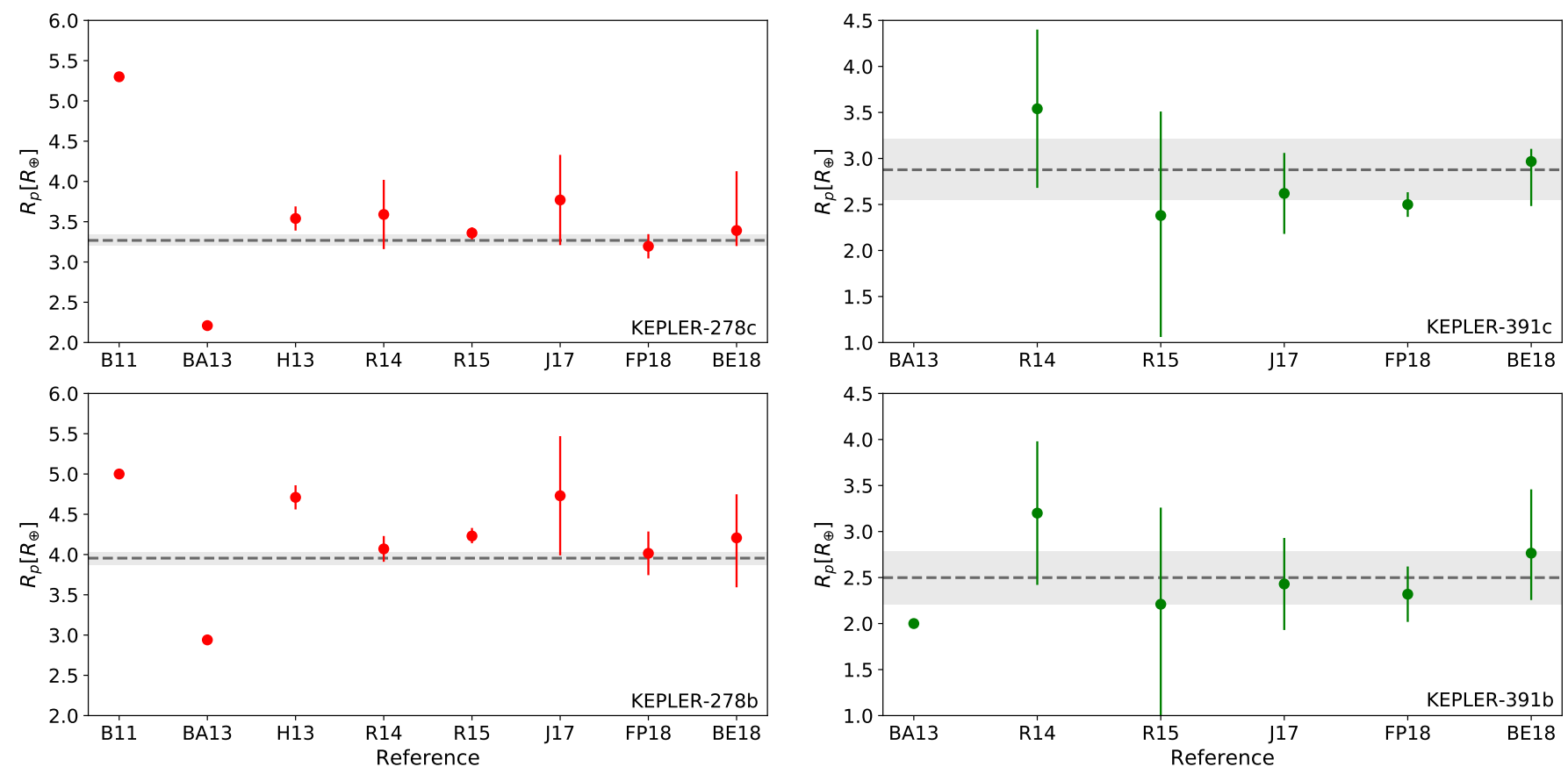

Fig. 11. Planetary radii derived in this study (dashed lines) in comparison with those reported by Batalha et al. (2013, BA13), Huber et al. (2013, H13), Rowe et al. (2014, R14), Rowe et al. (2015, R15), Johnson et al. (2017, J17), Fulton \& Petigura (2018, FP18), Berger et al. (2018, BE18) indicated by red and green filled circles for Kepler-278b/c (left) and Kepler-391b/c (right), respectively. Grey shaded areas indicate $1 \sigma$ uncertainty in our results.

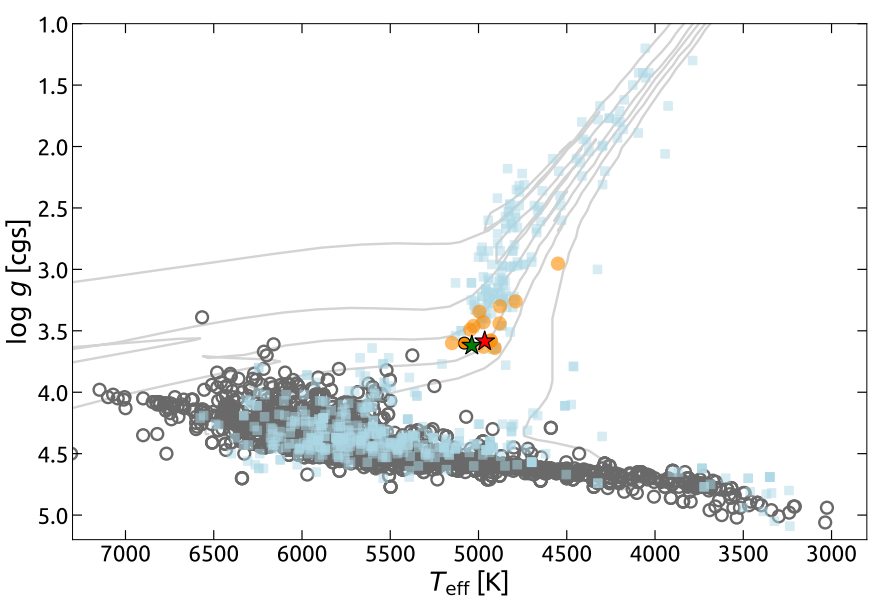

Fig. 12. Location of Kepler-278 (red star) and Kepler-391 (green star) in the HR diagram, based on the spectroscopic $T_{\text {eff }}$ and $\log g$ measured in this work, in comparison with other confirmed exoplanet hosts (taken from the NASA Exoplanet Archive on 2019 March 19; Akeson et al. 2013): light blue squares represent stars with confirmed planets detected via RVs, dark gray circles are stars with planets found by transits, and orange circles represent the small population of RGB stars with transiting planets. The star TOI-197, with values taken from Huber et al. (2019), is indicated by an orange circle with black edge-color. Error bars are omitted for clarity. Evolutionary tracks, corresponding to masses of $3,2,1.6,1.3,1.0$, and $0.6 M_{\odot}$ (left to right) for $[\mathrm{Fe} / \mathrm{H}]=+0.0 \mathrm{dex}$, from Girardi et al. (2000) are overplotted with continuous lines.

better than $30 \%$. Planet structure models from Zeng et al. (2016) are also overplotted. In terms of mass and radius, Kepler-278c and especially Kepler-278b fall in relatively unpopulated regions of the mass-radius diagram. Although the large uncertainties in the planetary masses of Kepler-278b/c ( $50 \%)$ prevent us from

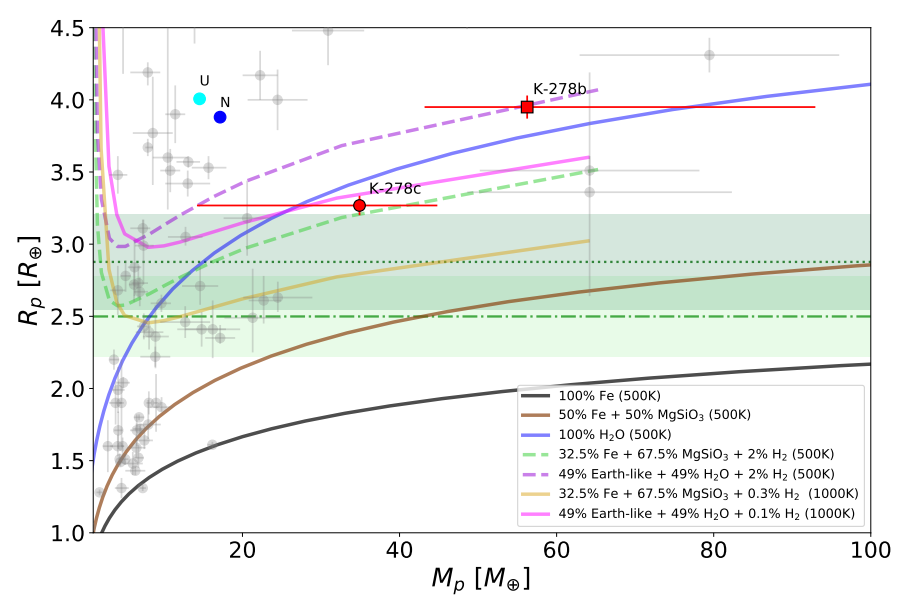

Fig. 13. Mass-radius diagram for all confirmed planets with masses between $1-100 M_{\oplus}$ and radii $1-4.5 R_{\oplus}$ determined with a precision better than $30 \%$ (data taken from the NASA Exoplanet Archive on 2019 June 10). Theoretical composition models from Zeng et al. (2016) are displayed with different lines and colors. Kepler-278b and Kepler-278c are indicated with a red square and circle. Kepler-391b and Kepler-391c are indicated with dark and light green bands, respectively, that represent the $1 \sigma$ uncertainty in our derived planetary radii. For reference, the solar system planets Uranus and Neptune are marked with a cyan and blue circle, respectively.

performing a detailed analysis of their composition, their radii are precise enough to locate both planets completely above the regime of solid planets with pure-iron or Earth-like rocky $(32.5 \%$ $\mathrm{Fe}+67.5 \% \mathrm{MgSiO}_{3}$ ) composition. Instead, both planets present bulk structures consistent with significant $\mathrm{H}_{2} \mathrm{O}$ or $\mathrm{H}_{2}$ content. In particular, Kepler-278b/c span a regime in mass in which they may either have a water-rich core $(49.95 \%$ Earth-like rocky core $+49.95 \% \mathrm{H}_{2} \mathrm{O}$ layer) with a $\mathrm{H}_{2}$-dominated gas envelope 
( $\sim 2 \%$ for both planets or $0.1 \%$ for Kepler-278c) or being pure $\mathrm{H}_{2} \mathrm{O}$ planets. For Kepler-278c given our uncertainties, the mass and radius also match the regime of an Earth-like rocky core with a $2 \% \mathrm{H}_{2}$ gaseous envelope. Improved measurements of the planetary masses for Kepler-278b/c would better constrain their interior compositions. Future studies, beyond the scope of this paper, like a full probabilistic Bayesian inference analysis would require precise $\mathrm{RV}$ s combined with the precise planetary radii and the stellar abundances obtained here (see, e.g., Dorn et al. 2017a,b; Almenara et al. 2018a).

For the Kepler-391 system, given the lack of TTV signals, we cannot estimate the planetary masses and therefore both planets are indicated in Fig. 13 with bands corresponding to their radii. However, considering the predicted masses from the massradius relation of Chen \& Kipping $\left(2017,6.74_{-3.27}^{+4.35} M_{\oplus}\right.$ for Kepler-391b and $8.92+6.22{ }_{-4.47} M_{\oplus}$ for Kepler-391c), these planets also might fall into the composition regimes consistent with a significant amount of water content or Earth-like rocky core and the presence of $\mathrm{H}_{2}$ gaseous envelopes.

\subsection{Binary companion candidate to Kepler-278}

It is worth mentioning that, using adaptive-optics imaging with the NIRC2 facility on the Keck II telescope, Kraus et al. (2016) detected a faint visual companion to Kepler-278 with a separation of 1'.984 \pm 0 .'050, projected separation of $\sim 860 \mathrm{AU}$, and position angle PA $10^{\mathrm{d}} 1 \pm 0^{\mathrm{d}} .11$. Based on star count models and the computed magnitude difference of $\Delta K=9.83 \pm 0.13$ relative to Kepler-278, Kraus et al. (2016) determined that this faint object is likely a bound companion given the low chance of background stars alignment. Nevertheless, they caution that multi-epoch imaging and common proper motion analysis are still necessary to conclusively confirm this companion is actually bound. Unfortunately, the visual companion is not resolved by Gaia.

The large 4" pixel scale of Kepler implies that it is not possible to isolate the photometry of Kepler-278 from that of its potential close secondary companion, whose extra flux might contribute to dilute the observed transit depth, and therefore the derived planetary radii. However, given the large magnitude difference $\Delta K$, we find that transit depths are insignificantly diluted by the light of the stellar companion and therefore the planetary radii do not require any correction ${ }^{28}$. This is also in agreement with the results of Furlan et al. (2017). Moreover, considering the low mass inferred for the stellar companion $\left(\sim 0.1 M_{\odot}\right.$; Kraus et al. 2016) and the transit duration of Kepler-278b ( $7.5 \mathrm{~h})$ and Kepler-278c $(\sim 11.3 \mathrm{~h})$, we determine that the planets are actually transiting the late subgiant/early red giant star and not the low-mass visual companion.

Considering the angular separation of the companion candidate $(\sim 2$ arcsec $)$, the difference in magnitudes between both stars ( $\Delta K=9.83$ ), and that our spectroscopic observations were taken with a seeing of $\sim 0.8 \operatorname{arcsec}^{29}$, we expect no significant contamination by the light of the companion candidate on the spectra of Kepler-278. Also, the spectra show no indication of a second set of stellar lines, which rules out additional close companions or background stars. Therefore, the existence of the visual companion should not affect any of the measurements performed from the GRACES spectra of Kepler-278.

28 The corrected planet radius can be obtained as $R_{\mathrm{p}, \mathrm{corr}}=$ $R_{\mathrm{p}} \sqrt{1+10^{-0.4 \Delta m}}$, where $\Delta m$ is the difference in magnitudes between the secondary and the primary (Furlan et al. 2017)

29 The on-sky size of the GRACES' fiber is 1.2 arcsec.

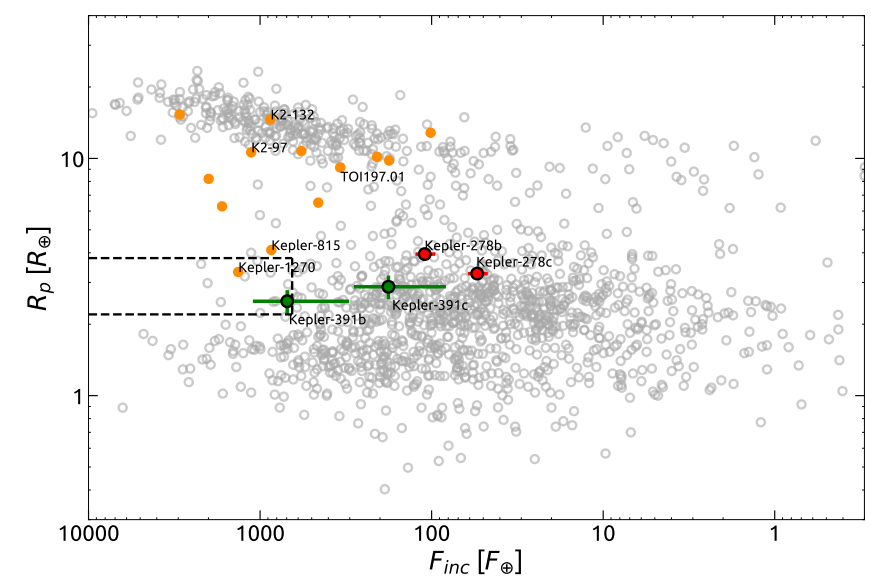

Fig. 14. Planet radius versus incident flux for transiting confirmed exoplanets (grey circles; data taken from the NASA Exoplanet Archive on 2019 March 19). Planets around Kepler-278 and Kepler-391 are highlighted in red and green circles, respectively. Planets around RGB stars are indicated with filled orange circles. The black dashed lines delimit the super-Earth desert as derived in Lundkvist et al. (2016). Other planets around RGB stars are also labelled (see text for more details).

\subsection{Planet properties in context}

Using the derived planetary parameters listed in Table 2, in Fig. 14 we located Kepler-278b/c and Kepler-391b/c in the radius-flux diagram. According to the classification scheme of Petigura et al. (2018) based on planetary radius and orbital period, Kepler-278b $\left(P=30.1 \mathrm{~d}, R_{\mathrm{p}}=3.95 R_{\oplus}\right)$, Kepler-278c $\left(P=51 \mathrm{~d}, R_{\mathrm{p}}=3.26 R_{\oplus}\right)$, and Kepler-391c $(P=20.5 \mathrm{~d}$ and $R_{\mathrm{p}}=2.88 R_{\oplus}$ ) would be classified as warm sub-Neptunes, whilst Kepler-391b $\left(P=7.4 \mathrm{~d}\right.$ and $\left.R_{\mathrm{p}}=2.49 R_{\oplus}\right)$ would be termed as a hot sub-Neptune. Only other two multi-planet systems have been reported to transit around RGB stars, Kepler-56 and Kepler-432, although they are composed by Jupiter-size planets and only the inner planet transits in the Kepler-432 system. Moreover, as can be seen in Fig. 14, the planets around Kepler-391 are the smallest planets $\left(R_{\mathrm{p}} \lesssim 3 R_{\oplus}\right)$ detected around stars ascending the RGB so far.

As evidenced on Fig. 14, Kepler-278b, Kepler-278c, and Kepler-391c fall outside the limits of the hot super-Earth "desert" (Lundkvist et al. 2016; Berger et al. 2018), whilst Kepler-391b resides within the rightmost boundary of this region. In this area there is a deficit of super-Earth to Neptune-size planets $\left(2.2 R_{\oplus}<\right.$ $\left.R_{\mathrm{p}}<3.8 R_{\oplus}\right)$ at high irradiance $\left(F_{\text {inc }}>650 F_{\oplus}\right)$, that could be a consequence of the photoevaporation of low-mass planets atmospheres (e.g., Owen \& Wu 2016). Although the errorbar in the $F_{\text {inc }}$ might still locate Kepler-391b slightly outside the rightmost limit, as the host star continues its ascent on the RGB the significant increase of $F_{\text {inc }}$ will move the position of Kepler-391b well inside this desert in just a few Myr. During this phase, a potential hydrogen atmosphere in Kepler-391b, which is likely given its radius and predicted mass (see Sect. 5.3), could be stripped by photoevaporation (Owen \& Wu 2017). With similar parameters to those of Kepler-391b, the planets around Kepler1270 and Kepler-815 might experience a similar process as their host stars evolve up the RGB.

On the other hand, planets such as those orbiting Kepler-391 and Kepler-278 are probably too small or too far from their hosts to fall into the regime of significant inflation (e.g., Van Eylen et al. 2016). For example, with $F_{\text {inc }} \sim 340 F_{\oplus}$ and $R_{\mathrm{p}}=9.16 R_{\text {Jup }}$, the planet TOI-197.01 (Huber et al. 2019) is located at the base of a "inflation sequence", a region in the radius-incident flux 
diagram (Fig. 14) from which the planet radius increases with stellar irradiation (see, e.g., Demory \& Seager 2011). While TOI-197.01 would just be starting to be re-inflated (Huber et al. 2019), there are other planets around RGB stars on this inflation sequence, including the giant planets K2-97b and K2-132b whose radii have already become re-inflated as result of stellar evolution (Grunblatt et al. 2016, 2017).

Planets Kepler-278b/c and Kepler-391b/c are part of the small group of planets $(\sim 20)$, most of them detected via transits, in close-in orbits around evolved stars with a semi-major axis below $\sim 0.5$ AU $(P \lesssim 100 \mathrm{~d})$. One of the main scenarios to explain the paucity of close-in planets considers that these objects might end up engulfed by their host stars as they ascend on the RGB (e.g., Villaver \& Livio 2009). Based on our computed $T_{\text {eff }},[\mathrm{Fe} / \mathrm{H}], R_{\star}$, and $M_{\star}$ values, listed on Table 1 , and evolutionary tracks from Girardi et al. (2000), we found that the stellar surface of Kepler-391 will reach the inner planet in $\sim 410 \mathrm{Myr}\left(\approx 17 R_{\odot}\right)$ and the outer planet in $428 \mathrm{Myr}\left(\approx 63 R_{\odot}\right)$. Kepler-278, that is slightly more evolved than Kepler-391, will engulf its inner planet in $\sim 408 \mathrm{Myr}\left(\approx 43 R_{\odot}\right)$ and in $\sim 428 \mathrm{Myr}$ $\left(\approx 63 R_{\odot}\right)$ the outer planet. These values represent a conservative upper limit on the remaining lifetimes of the planets, because the engulfment could be accelerated due to tidal interactions in the star-planet systems causing the orbital decay of the planets (Villaver \& Livio 2009; Matsumura et al. 2010; Kunitomo et al. 2011).

\subsection{Stellar abundances and planets}

The stellar metallicities that we measured for Kepler-278 $([\mathrm{Fe} / \mathrm{H}]=0.22 \mathrm{dex})$ and Kepler-391 $([\mathrm{Fe} / \mathrm{H}]=0.04 \mathrm{dex})$, from our GRACES spectra, are in line with the tendency that small planets can occur around stars with a wide range of metallicities (Buchhave et al. 2012, 2014; Petigura et al. 2018). Interestingly, the higher metallicity of Kepler-278 in comparison with that of Kepler-391 agrees with the trend of increasing planet radius with the host star metallicity (Petigura et al. 2018). On the other hand, recent studies suggest that $[\mathrm{Mg} / \mathrm{Si}]$ mineralogical ratio probably plays an important role in the formation of small planets, since they tend to orbit stars with larger $[\mathrm{Mg} / \mathrm{Si}]$ ratios in comparison with Jovian host stars or control stars without detected planets (Adibekyan et al. 2015; Mack et al. 2018). The relatively low mineralogical ratio that we found for both stars $([\mathrm{Mg} / \mathrm{Si}]=$ 0.00 for Kepler-278 and $[\mathrm{Mg} / \mathrm{Si}]=-0.03$ for Kepler-391) seems to point out that these evolved stars do not follow the reported trend, although we note that in Adibekyan et al. (2015) planets are categorized by mass rather than radius and most of them are single systems around dwarf stars. To make a proper comparison, it would be interesting to see how the results for these evolved Kepler stars are compared with the $[\mathrm{Mg} / \mathrm{Si}]$ ratios of a larger sample of evolved stars with planets. Considering that, to date, most of RGB stars host giant RV planets, this sample would represent a good starting point for such a comparison.

\subsection{Eccentricity of the planets orbiting Kepler-278}

The highly eccentric orbits of Kepler-278b $\left(e=0.696_{-0.026}^{+0.017}\right)$ and Kepler-278c $\left(e=0.616_{-0.023}^{+0.015}\right)$ revealed by our photodynamical analysis are extremely rare among the known multi-planet systems and therefore add further interest to this system. The only previous measurement of eccentricity in this system was performed by Van Eylen \& Albrecht (2015) based on a technique relying on Kepler's second law, measuring the duration of individual transits from Kepler data and using the asteroseismic stellar density. After removing the TTV signal detected for the outer planet, using a sinusoidal model, they also found an eccentric orbit with a mode of $e=0.51$ and a $68 \%$ confidence interval $[0.39,0.70]$. The authors, however, advise caution about this result due to the large degeneracy with the impact parameter and the poor quality of the transit light curves. For Kepler-278b, on the other hand, they found a circular orbit (modal value of $e=0.03$ and $68 \%$ confidence interval at $[0,0.36])$ but did not detect a TTV signal, which might have biased their result for this planet.

The striking eccentricities found for Kepler-278b/c require additional and deeper studies. A high-precision radial velocity follow-up of Kepler-278 is necessary to confirm the eccentricities found in our analysis. From our computed orbital periods, planetary masses, and eccentricities, we estimate a RV semi-

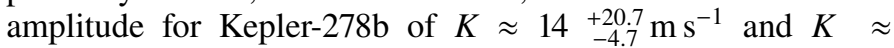
$6.6_{-5.1}^{+2.3} \mathrm{~m} \mathrm{~s}^{-1}$ for Kepler-278c. Considering that Kepler-278 is not too faint $(V=11.8 \mathrm{mag})$, is a slow rotator $\left(v \sin i=2.5 \mathrm{~km} \mathrm{~s}^{-1}\right)$, and that our estimated RV jitter is $4.3 \mathrm{~m} \mathrm{~s}^{-1}$, the RV signal for Kepler-278b might be detectable with existing precise instruments such as Keck-HIRES.

If future studies indeed support high-eccentric orbits, it will be necessary to perform a detailed dynamical study of this system in order to constrain its origin and evolution. With the eccentricity values found here, Kepler-278 would join the short list of systems where both planets present significant eccentricities such as those around Kepler-432 (Quinn et al. 2015). Additionally, they would be among the most eccentric planets around evolved stars. Kepler-278b/c would not follow the trend showing that small planets in Kepler multi-planet have low eccentricities (Van Eylen \& Albrecht 2015; Van Eylen et al. 2019; Mills et al. 2019). In contrast, such values would be in line with the eccentric orbits found for close-in giant planets orbiting evolved stars (Grunblatt et al. 2018), for which orbital decay happens faster than tidal circularization due to tides raised on the evolved host stars (Villaver \& Livio 2009; Villaver et al. 2014; Grunblatt et al. 2018).

Nevertheless, considering the masses, orbital periods and highly eccentric orbits of Kepler-278b/c, it is possible that other mechanisms be at work. The large eccentricities could be the result of planet-planet scattering events (e.g., Rasio \& Ford 1996; Ford \& Rasio 2008). However, since in this case it would be expected that both the eccentricities and the mutual inclinations be high, the coplanar nature of this system presents a puzzle for this scenario. Moreover, given the semi-major axis and masses of the planets around Kepler-278, a coplanar high eccentricity migration mechanism (Petrovich 2015) would not be possible for this system ${ }^{30}$. Another possible scenario might involve the influence of a stellar binary companion, like the one detected in the adaptive-optics images (see Sect. 5.4), inducing secular Lidov-Kozai cycles (Lidov 1962; Kozai 1962). In this case, the external fourth body should be highly inclined with respect to the orbital plane in order to maintain the mutual inclinations of the planetary orbits (Takeda et al. 2008a; Almenara et al. 2018b). Additional scenarios that could produce coplanar highly-eccentric orbits (e.g., spin-orbit coupling, collisions; see Almenara et al. 2018b), also should be considered.

Further information that might be key to understand the origin of this system is whether the stellar spin and the orbits of

\footnotetext{
${ }^{30}$ One of the configurations in which the coplanar, and high eccentricity migration mechanism could operate is when the two planets present $e \gtrsim$ 0.5 and $M_{\mathrm{p}, \text { in }} / M_{\mathrm{p}, \text { out }}\left(a_{\mathrm{in}} / a_{\mathrm{out}}\right)^{1 / 2} \lesssim 0.16$ (Petrovich 2015), where $M_{\mathrm{p}}$ is the planetary mass, $a$ is the semi-major axis, and the indices in and out refer to the inner and outer planet, respectively. However, for the planets in Kepler-278 this expression is equal to $\sim 1.38$.
} 
planets are aligned. Although we have derived the rotational velocity, unfortunately, no rotational period has been found for this star from Kepler data (McQuillan et al. 2013; Mazeh et al. 2015), which could provide this information. Alternatively, the analysis of the rotational splitting of asteroseismic oscillation modes of the available Kepler data and those forthcoming from TESS $^{31}$ could be used to determine the stellar obliquity, similar to Huber et al. (2013) and Quinn et al. (2015). This information, coupled with a detailed dynamical study of this system, which is beyond the scope of the current paper, would be key to further constrain the formation, evolution, and stability of the Kepler-278 system.

\section{Summary and conclusions}

In this paper, we have performed a detailed stellar and planetary characterization study of the remarkable multi-planet systems Kepler-278 and Kepler-391. Our main conclusions are summarized as follows:

- Using high-quality spectra collected with Gemini-GRACES, we have refined the stellar parameters $\left(T_{\mathrm{eff}}, \log g, v \sin i\right.$, $\log \left(R_{\mathrm{HK}}^{\prime}\right), M_{\star}, R_{\star}$, and $\left.\tau_{\star}\right)$, and derived precise chemical abundances of 25 elements (Li, C, N, O, Na, Mg, Al, Si, S, $\mathrm{Ca}, \mathrm{Sc}, \mathrm{Ti}, \mathrm{V}, \mathrm{Cr}, \mathrm{Mn}, \mathrm{Fe}, \mathrm{Co}, \mathrm{Ni}, \mathrm{Cu}, \mathrm{Zn}, \mathrm{Sr}, \mathrm{Y}, \mathrm{Zr}, \mathrm{Ba}$, and $\mathrm{Ce})$. Nine of these elements and the carbon isotopic ratios, ${ }^{12} \mathrm{C} /{ }^{13} \mathrm{C}$, were not previously measured. Overall, our stellar parameters agree reasonably well with most of the previous results. However, we find that Kepler-278 is 15\% less massive than recently reported (Mathur et al. 2017; Johnson et al. 2017; Fulton \& Petigura 2018).

- Both the stellar activity index based on the Ca II H \& K lines and that obtained from the $\mathrm{Ca}$ II infrared triplet lines indicate low chromospheric activity for both stars.

- The stellar parameters of Kepler-278 and Kepler-391 along with their measured carbon ${ }^{12} \mathrm{C} /{ }^{13} \mathrm{C}$ isotopic ratio reveal that both stars are just starting their ascent on the RGB. For Kepler-278, this is also confirmed from asteroseismic data.

- The chemical abundances of light, alpha, Fe-peak, and heavy elements of both stars follow the abundance trends of other evolved thin disk stars in the solar neighborhood. Also, the abundance vs. condensation temperature slopes for both stars are consistent with the average value presented by M16 for subgiants with planets.

- The lithium abundance of Kepler-391, $A(\mathrm{Li})_{\mathrm{NLTE}}=1.29 \pm$ 0.09 dex, is slightly below the standard limit of the rare Lirich giant stars. The evolutionary state of this star, its low $v \sin i$, and lack of other chemical peculiarities would support that the Li content of Kepler-391 is most likely a remnant from the main-sequence phase and not the consequence of a recent planetary engulfment episode or a fresh lithium production phase.

- The measured stellar metallicities for Kepler-278 $([\mathrm{Fe} / \mathrm{H}]=$ 0.22 dex $)$ and Kepler-391 $([\mathrm{Fe} / \mathrm{H}]=0.04$ dex $)$ are consistent with the tendency that small planets can occur around stars with a wide range of metallicities (e.g., Petigura et al. 2018). Also, according to the relatively low mineralogical ratio obtained for both stars $([\mathrm{Mg} / \mathrm{Si}] \sim 0)$, none of them would seem to follow the trend of higher $[\mathrm{Mg} / \mathrm{Si}]$ ratios for hosts with small planets (Adibekyan et al. 2015).

- Using a photodynamical analysis of the Kepler light curves, in combination with our new stellar parameters, we derived

31 The Transiting Exoplanet Survey Satellite (TESS; Ricker 2015) will observe Kepler-278 between July 18 and August 15 in 2019. improved planetary properties. In particular, for the Kepler278 system, the increased precision in the transit times obtained from this analysis allowed us to measure for the first time the masses of Kepler-278b $\left(M_{\mathrm{p}}=56_{-13}^{+37} M_{\oplus}\right)$ and Kepler-278c $\left(M_{\mathrm{p}}=35_{-21}^{+9.9} M_{\oplus}\right)$. Not only do we confirm the presence of a TTV signal in Kepler-278c, but also detect a previously unreported TTV signal in the inner planet Kepler-278b. For the system Kepler-391, given the lack of detected TTV signals, only upper limits on the masses could be provided.

- The location of Kepler-278b/c in the mass-radius diagram suggests that their bulk structures might be consistent with significant amount of water content and the presence of $\mathrm{H}_{2}$ gaseous envelopes. According to their radii and orbital period, Kepler-278b, Kepler-278c, and Kepler-391c would be classified as warm sub-Neptunes. Kepler-391b is a warm sub-Neptune that resides just inside the rightmost boundary of the hot super-Earth desert (Lundkvist et al. 2016). In case this planet has a bulk structure consistent with a significant amount of water content or $\mathrm{H}_{2}$ gaseous envelope, this may be suffering photoevaporation. It is expected that this process increases in a few Myr as its host star continues ascending on the RGB.

- The photodynamical analysis reveals that the orbits of both planets around Kepler-278 are surprisingly eccentric $(e \sim$ 0.7 ) and coplanar, which pose a puzzle about its origin. A precise RV follow-up of Kepler-278 is needed to confirm the eccentricity values presented here.

Finally, we note that Kepler-278b/c and Kepler-391b/c are part of the small sample $(\sim 15)$ of close-in $(a<0.5$ AU) transiting planets orbiting RGB stars. Kepler-278 and Kepler-391 are also exceptional because they are two of the three multi-planet systems detected to date that transit evolved stars. Moreover, the planets orbiting Kepler-278 and Kepler-391 are among the smallest bodies discovered so far around RGB stars.

The fact that Kepler observed $\sim 16000$ red giants (Yu et al. 2018) but only a handful of planets were found highlighting the difficulty of finding transiting planets around evolved stars, especially on the RGB. Although this observational bias likely may contribute to the relative paucity of close-in planets detected around giants, it is necessary to examine larger samples of stars in this evolutionary stage in order to constrain other proposed scenarios such as planet engulfment or different planet-formation mechanisms. The TESS mission will observe hundreds of thousands of red giants (with $T_{\text {mag }}<11$ ) from which is expected the detection of 50-100 new planets (Campante et al. 2016; Barclay et al. 2018) that would be more amenable to spectroscopic and photometric follow-up. A larger sample of planets transiting evolved stars on the RGB, will provide better constraints on the formation and evolution of planets around intermediate and high-mass stars. Moreover, since the expected TESS detection limit in planetary mass is approximately the mass of Neptune (Campante et al. 2016), TESS might give us some clues about the frequency of systems similar to Kepler-278 and Kepler-391.

Acknowledgements. This study has been partially supported by UNAM-PAPIIT IN-107518. E.J. and R.P. acknowledge the financial support from DGAPA in the forms of Post-Doctoral Fellowships. We thank the anonymous referee for helpful comments and suggestions that improved the paper. This publication has made use of VOSA, developed under the Spanish Virtual Observatory project supported from the Spanish MINECO through grant AyA2017-84089. This research has made use of the NASA Exoplanet Archive, which is operated by the California Institute of Technology, under contract with the National Aeronautics and Space Administration under the Exoplanet Exploration Program. This research has made use of the SIMBAD database, operated at CDS, Strasbourg, 
France. This paper includes data collected by the Kepler mission. Funding for the Kepler mission is provided by the NASA Science Mission directorate. Data presented in this paper were obtained from the Mikulski Archive for Space Telescopes (MAST). Software. IRAF, OPERA (Martioli et al. 2012), MOOG (Sneden 1973), $q^{2}$ (Ramírez et al. 2014), Teff-LR (Sousa et al. 2010), ARES (Sousa et al. 2015), VOSA (Bayo et al. 2008), iSpec (Blanco-Cuaresma et al. 2014), PARAM (da Silva et al. 2006), INSPECT (Lind et al. 2009), REBOUND (Rein \& Liu 2012), emcee (Goodman \& Weare 2010; Foreman-Mackey et al. 2013), and WHFast (Rein \& Tamayo 2015)

\section{References}

Adamów, M., Niedzielski, A., Villaver, E., Nowak, G., \& Wolszczan, A. 2012, ApJ, 754, L15

Adamów, M., Niedzielski, A., Villaver, E., Wolszczan, A., \& Nowak, G. 2014, A\&A, 569, A55

Adamów, M., Niedzielski, A., Villaver, E., et al. 2015, A\&A, 581, A94

Adibekyan, V. Z., Benamati, L., Santos, N. C., et al. 2015, MNRAS, 450, 1900

Afşar, M., Sneden, C., \& For, B.-Q. 2012, AJ, 144, 20

Aguilera-Gómez, C., Chanamé, J., Pinsonneault, M. H., \& Carlberg, J. K. 2016, ApJ, 829, 127

Akeson, R. L., Chen, X., Ciardi, D., et al. 2013, PASP, 125, 989

Allard, F., Homeier, D., \& Freytag, B. 2012, Phil. Trans. R. Soc. London, Ser. A, 370,2765

Almenara, J. M., Díaz, R. F., Mardling, R., et al. 2015, MNRAS, 453, 2644

Almenara, J. M., Díaz, R. F., Dorn, C., Bonfils, X., \& Udry, S. 2018a, MNRAS 478,460

Almenara, J. M., Díaz, R. F., Hébrard, G., et al. 2018b, A\&A, 615, A90

Arenou, F., Grenon, M., \& Gomez, A. 1992, A\&A, 258, 104

Asplund, M., Grevesse, N., Sauval, A. J., \& Scott, P. 2009, ARA\&A, 47, 481

Baliunas, S. L., Donahue, R. A., Soon, W. H., et al. 1995, ApJ, 438, 269

Barclay, T., Pepper, J., \& Quintana, E. V. 2018, ApJS, 239, 2

Barros, S. C. C., Boué, G., Gibson, N. P., et al. 2013, MNRAS, 430, 3032

Bastien, F. A., Stassun, K. G., \& Pepper, J. 2014, ApJ, 788, L9

Bastien, F. A., Stassun, K. G., Basri, G., \& Pepper, J. 2016, ApJ, 818, 43

Batalha, N. M., Rowe, J. F., Bryson, S. T., et al. 2013, ApJS, 204, 24

Bauschlicher, C. W. J., Langhoff, S. R., \& Taylor, P. R. 1988, ApJ, 332, 531

Bayo, A., Rodrigo, C., Barrado Y Navascués, D., et al. 2008, A\&A, 492, 277

Bedell, M., Meléndez, J., Bean, J. L., et al. 2014, ApJ, 795, 23

Berger, T. A., Howard, A. W., \& Boesgaard, A. M. 2018, ApJ, 855, 115

Blanco-Cuaresma, S., Soubiran, C., Heiter, U., \& Jofré, P. 2014, A\&A, 569, A111

Borucki, W. J., Koch, D., Basri, G., et al. 2010, Science, 327, 977

Borucki, W. J., Koch, D. G., Basri, G., et al. 2011, ApJ, 736, 19

Bressan, A., Marigo, P., Girardi, L., et al. 2012, MNRAS, 427, 127

Brewer, J. M., \& Fischer, D. A. 2018, ApJS, 237, 38

Brown, T. M., Gilliland, R. L., Noyes, R. W., \& Ramsey, L. W. 1991, ApJ, 368, 599

Brown, T. M., Latham, D. W., Everett, M. E., \& Esquerdo, G. A. 2011, AJ, 142 112

Bruntt, H., Deleuil, M., Fridlund, M., et al. 2010, A\&A, 519, A51

Bruntt, H., Frandsen, S., \& Thygesen, A. O. 2011, A\&A, 528, A121

Bruntt, H., Basu, S., Smalley, B., et al. 2012, MNRAS, 423, 122

Buchhave, L. A., Latham, D. W., Johansen, A., et al. 2012, Nature, 486, 375

Buchhave, L. A., Bizzarro, M., Latham, D. W., et al. 2014, Nature, 509, 593

Burkert, A., \& Ida, S. 2007, ApJ, 660, 845

Busà, I., Aznar Cuadrado, R., Terranegra, L., Andretta, V., \& Gomez, M. T. 2007, A\&A, 466, 1089

Cameron, A. G. W., \& Fowler, W. A. 1971, ApJ, 164, 111

Campante, T. L., Schofield, M., Kuszlewicz, J. S., et al. 2016, ApJ, 830, 138

Carlberg, J. K., Cunha, K., Smith, V. V., \& Majewski, S. R. 2012, ApJ, 757, 109

Carlos, M., Nissen, P. E., \& Meléndez, J. 2016, A\&A, 587, A100

Carter, J. A., Fabrycky, D. C., Ragozzine, D., et al. 2011, Science, 331, 562

Casagrande, L., Ramírez, I., Meléndez, J., Bessell, M., \& Asplund, M. 2010, A\&A, 512, A54

Castelli, F., \& Kurucz, R. L. 2003, IAU Symp., 210, A20

Chambers, K. C., Magnier, E. A., Metcalfe, N., et al. 2016, ArXiv e-prints [arXiv: 1612.05560 ]

Chaplin, W. J., Elsworth, Y., Davies, G. R., et al. 2014, MNRAS, 445, 946

Chavero, C., de La Reza, R., Domingos, R. C., et al. 2010, A\&A, 517, A40

Chen, J., \& Kipping, D. 2017, ApJ, 834, 17

Chene, A.-N., Padzer, J., Barrick, G., et al. 2014, SPIE Conf. Ser., 9151, 47

Choi, J., Dotter, A., Conroy, C., et al. 2016, ApJ, 823, 102

Chontos, A., Huber, D., Latham, D. W., et al. 2019, AJ, 157, 192

Christensen-Dalsgaard, J. 2008, Ap\&SS, 316, 13

Currie, T. 2009, ApJ, 694, L171

da Silva, L., Girardi, L., Pasquini, L., et al. 2006, A\&A, 458, 609

da Silva, R., Milone, A. C., \& Reddy, B. E. 2011, A\&A, 526, A71 de Medeiros, J. R., Da Rocha, C., \& Mayor, M. 1996, A\&A, 314, 499

Delgado Mena, E., Tsantaki, M., Adibekyan, V. Z., et al. 2017, A\&A, 606, A94

Demarque, P., Guenther, D. B., Li, L. H., Mazumdar, A., \& Straka, C. W. 2008, Ap\&SS, 316, 31

Demarque, P., Woo, J.-H., Kim, Y.-C., \& Yi, S. K. 2004, ApJS, 155, 667

Demory, B.-O., \& Seager, S. 2011, ApJS, 197, 12

Döllinger, M. P., Hatzes, A. P., Pasquini, L., Guenther, E. W., \& Hartmann, M. 2009, A\&A, 505, 1311

Dorn, C., Hinkel, N. R., \& Venturini, J. 2017a, A\&A, 597, A38

Dorn, C., Venturini, J., Khan, A., et al. 2017b, A\&A, 597, A37

Dotter, A., Chaboyer, B., Jevremović, D., et al. 2008, ApJS, 178, 89

Doyle, A. P., Smalley, B., Faedi, F., Pollacco, D., \& Gómez Maqueo Chew, Y. 2017, MNRAS, 469, 4850

Egeland, R., Soon, W., Baliunas, S., et al. 2017, ApJ, 835, 25

Enoch, B., Collier Cameron, A., Parley, N. R., \& Hebb, L. 2010, A\&A, 516, A33

Feltzing, S., Howes, L. M., McMillan, P. J., \& Stonkute, E. 2017, MNRAS, 465 L109

Fischer, D. A., \& Valenti, J. 2005, ApJ, 622, 1102

Ford, E. B., \& Rasio, F. A. 2008, ApJ, 686, 621

Foreman-Mackey, D., Hogg, D. W., Lang, D., \& Goodman, J. 2013, PASP, 125, 306

Frasca, A., Molenda-Żakowicz, J., De Cat, P., et al. 2016, A\&A, 594, A39

Fulton, B. J., \& Petigura, E. A. 2018, AJ, 156, 264

Furlan, E., Ciardi, D. R., Everett, M. E., et al. 2017, AJ, 153, 71

Gai, N., Basu, S., Chaplin, W. J., \& Elsworth, Y. 2011, ApJ, 730, 63

Gaia Collaboration (Brown, A. G. A., et al.) 2018, A\&A, 616, A1

Gautier, III, T. N., Batalha, N. M., Borucki, W. J., et al. 2010, ArXiv e-prints [arXiv:1001.0352]

Ghezzi, L., Cunha, K., Schuler, S. C., \& Smith, V. V. 2010a, ApJ, 725, 721

Ghezzi, L., Cunha, K., Smith, V. V., \& de la Reza, R. 2010b, ApJ, 724, 154

Ghezzi, L., Montet, B. T., \& Johnson, J. A. 2018, ApJ, 860, 109

Gilroy, K. K., \& Brown, J. A. 1991, ApJ, 371, 578

Girardi, L., Bressan, A., Bertelli, G., \& Chiosi, C. 2000, A\&AS, 141, 371

Gonzalez, G. 1997, MNRAS, 285, 403

Gonzalez, G., \& Vanture, A. D. 1998, A\&A, 339, L29

González Hernández, J. I., Delgado-Mena, E., Sousa, S. G., et al. 2013, A\&A, 552, A6

Goodman, J., \& Weare, J. 2010, Comm. App. Math. Comp. Sci., 5, 65

Grunblatt, S. K., Huber, D., Gaidos, E. J., et al. 2016, AJ, 152, 185

Grunblatt, S. K., Huber, D., Gaidos, E., et al. 2017, AJ, 154, 254

Grunblatt, S. K., Huber, D., Gaidos, E., et al. 2018, ApJ, 861, L5

Gustafsson, B., Edvardsson, B., Eriksson, K., et al. 2008, A\&A, 486, 951

Hekker, S., \& Meléndez, J. 2007, A\&A, 475, 1003

Hekker, S., Elsworth, Y., Mosser, B., et al. 2013, A\&A, 556, A59

Henden, A. A., Levine, S., Terrell, D., \& Welch, D. L. 2015, AAS Meeting Abstracts, 225, 336.16

Hinkel, N. R., Young, P. A., Pagano, M. D., et al. 2016, ApJS, 226, 4

Hodapp, K. W., Kaiser, N., Aussel, H., et al. 2004, Astron. Nachr., 325, 636

Holczer, T., Mazeh, T., Nachmani, G., et al. 2016, ApJS, 225, 9

Huber, K. P., \& Herzberg, G. 1979, Molecular Spectra and Molecular Structure (Berlin: Springer)

Huber, D., Chaplin, W. J., Christensen-Dalsgaard, J., et al. 2013, ApJ, 767, 127

Huber, D., Silva Aguirre, V., Matthews, J. M., et al. 2014, ApJS, 211, 2

Huber, D., Zinn, J., Bojsen-Hansen, M., et al. 2017, ApJ, 844, 102

Huber, D., Chaplin, W. J., Chontos, A., et al. 2019, AJ, 157, 245

Isaacson, H., \& Fischer, D. 2010, ApJ, 725, 875

Jofré, E., Saffe, C., \& Petrucci, R. 2010, Boletin de la Asociacion Argentina de Astronomia La Plata Argentina, 53, 273

Jofré, E., Petrucci, R., García, L., \& Gómez, M. 2015a, A\&A, 584, L3

Jofré, E., Petrucci, R., Saffe, C., et al. 2015b, A\&A, 574, A50

Jofré, P., Heiter, U., Worley, C. C., et al. 2017, A\&A, 601, A38

Johnson, J. A., Fischer, D. A., Marcy, G. W., et al. 2007, ApJ, 665, 785

Johnson, J. A., Aller, K. M., Howard, A. W., \& Crepp, J. R. 2010, PASP, 122, 905

Johnson, J. A., Petigura, E. A., Fulton, B. J., et al. 2017, AJ, 154, 108

Kallinger, T., Weiss, W. W., Barban, C., et al. 2010, A\&A, 509, A77

Kass, R. E., \& Raftery, A. E. 1995, J. Am. Stat. Assoc., 90, 773

Kepler Mission Team. 2009, VizieR Online Data Catalog: V/133

Kipping, D. M. 2010, MNRAS, 408, 1758

Kipping, D. M. 2013, MNRAS, 435, 2152

Kozai, Y. 1962, AJ, 67, 591

Kraus, A. L., Ireland, M. J., Huber, D., Mann, A. W., \& Dupuy, T. J. 2016, AJ, 152,8

Kretke, K. A., Lin, D. N. C., Garaud, P., \& Turner, N. J. 2009, ApJ, 690, 407

Kumar, Y. B., Reddy, B. E., \& Lambert, D. L. 2011, ApJ, 730, L12

Kunitomo, M., Ikoma, M., Sato, B., Katsuta, Y., \& Ida, S. 2011, ApJ, 737, 66

Kupka, F., Piskunov, N., Ryabchikova, T. A., Stempels, H. C., \& Weiss, W. W. 1999, A\&AS, 138, 119 
Kurucz, R., \& Bell, B. 1995, Atomic Line Data (Cambridge, MA: Smithsonian Astrophysical Observatory), 23

Lidov, M. L. 1962, Planet. Space Sci., 9, 719

Lillo-Box, J., Barrado, D., Henning, T., et al. 2014, A\&A, 568, L1

Lind, K., Asplund, M., \& Barklem, P. S. 2009, A\&A, 503, 541

Lind, K., Bergemann, M., \& Asplund, M. 2012, MNRAS, 427, 50

Lissauer, J. J., Marcy, G. W., Bryson, S. T., et al. 2014, ApJ, 784, 44

Lodders, K. 2003, ApJ, 591, 1220

Lopez, E. D., \& Fortney, J. J. 2016, ApJ, 818, 4

Lovis, C., Dumusque, X., Santos, N. C., et al. 2011, ArXiv e-prints [arXiv:1107.5325]

Lovis, C., \& Mayor, M. 2007, A\&A, 472, 657

Lovis, C., \& Pepe, F. 2007, A\&A, 468, 1115

Luck, R. E., \& Heiter, U. 2007, AJ, 133, 2464

Lundkvist, M. S., Kjeldsen, H., Albrecht, S., et al. 2016, Nat. Commun., 7, 11201

Luri, X., Brown, A. G. A., Sarro, L. M., et al. 2018, A\&A, 616, A9

Mack, C. E., Strassmeier, K. G., Ilyin, I., et al. 2018, A\&A, 612, A46

Maldonado, J., \& Villaver, E. 2016, A\&A, 588, A98

Maldonado, J., Villaver, E., \& Eiroa, C. 2013, A\&A, 554, A84

Mandel, K., \& Agol, E. 2002, ApJ, 580, L171

Manduca, A., Bell, R. A., \& Gustafsson, B. 1977, A\&A, 61, 809

Marigo, P., Girardi, L., Bressan, A., et al. 2008, A\&A, 482, 883

Martinez, C. F., Cunha, K., Ghezzi, L., \& Smith, V. V. 2019, ApJ, 875, 29

Martioli, E., Teeple, D., Manset, N., et al. 2012, SPIE Conf. Ser., 8451, 2

Mashonkina, L., Christlieb, N., Barklem, P. S., et al. 2010, A\&A, 516, A46

Mathur, S., Huber, D., Batalha, N. M., et al. 2017, ApJS, 229, 30

Matsumura, S., Peale, S. J., \& Rasio, F. A. 2010, ApJ, 725, 1995

Mazeh, T., Perets, H. B., McQuillan, A., \& Goldstein, E. S. 2015, ApJ, 801, 3

McQuillan, A., Mazeh, T., \& Aigrain, S. 2013, ApJ, 775, L11

Meléndez, J., Asplund, M., Gustafsson, B., \& Yong, D. 2009, ApJ, 704, L66

Meléndez, J., Bergemann, M., Cohen, J. G., et al. 2012, A\&A, 543, A29

Meléndez, J., Bedell, M., Bean, J. L., et al. 2017, A\&A, 597, A34

Miglio, A., Chiappini, C., Morel, T., et al. 2013, MNRAS, 429, 423

Mills, S. M., Howard, A. W., Petigura, E. A., et al. 2019, AJ, 157, 198

Mittag, M., Schmitt, J. H. M. M., \& Schröder, K.-P. 2013, A\&A, 549, A117

Molenda-Żakowicz, J., Latham, D. W., Catanzaro, G., Frasca, A., \& Quinn, S. N. 2011, MNRAS, 412, 1210

Morel, T., \& Miglio, A. 2012, MNRAS, 419, L34

Morel, T., Miglio, A., Lagarde, N., et al. 2014, A\&A, 564, A119

Mortier, A., Santos, N. C., Sousa, S. G., et al. 2013, A\&A, 558, A106

Mortier, A., Sousa, S. G., Adibekyan, V. Z., Brandão, I. M., \& Santos, N. C. 2014, A\&A, 572, A95

Morton, T. D. 2015, Astrophysics Source Code Library [record ascl:1503.010]

Morton, T. D., Bryson, S. T., Coughlin, J. L., et al. 2016, ApJ, 822, 86

Mosser, B., Benomar, O., Belkacem, K., et al. 2014, A\&A, 572, L5

Murray, N., \& Chaboyer, B. 2002, ApJ, 566, 442

Neves, V., Santos, N. C., Sousa, S. G., Correia, A. C. M., \& Israelian, G. 2009 A\&A, 497, 563

Niedzielski, A., Goździewski, K., Wolszczan, A., et al. 2009, ApJ, 693, 276

Nissen, P. E. 2015, A\&A, 579, A52

Nissen, P. E. 2016, A\&A, 593, A65

Noyes, R. W., Hartmann, L. W., Baliunas, S. L., Duncan, D. K., \& Vaughan, A. H. 1984, ApJ, 279, 763

Owen, J. E., \& Wu, Y. 2016, ApJ, 817, 107

Owen, J. E., \& Wu, Y. 2017, ApJ, 847, 29

Pasquini, L., Döllinger, M. P., Hatzes, A., et al. 2008, IAU Symp., 249, 209

Petigura, E. A., Howard, A. W., Marcy, G. W., et al. 2017, AJ, 154, 107

Petigura, E. A., Marcy, G. W., Winn, J. N., et al. 2018, AJ, 155, 89

Petrovich, C. 2015, ApJ, 805, 75

Pietrinferni, A., Cassisi, S., Salaris, M., \& Castelli, F. 2004, ApJ, 612, 168
Pinsonneault, M. H., An, D., Molenda-Żakowicz, J., et al. 2012, ApJS, 199, 30 Quinn, S. N., White, T. R., Latham, D. W., et al. 2015, ApJ, 803, 49

Ramírez, I., \& Allende Prieto, C. 2011, ApJ, 743, 135

Ramírez, I., \& Meléndez, J. 2005, ApJ, 626, 465

Ramírez, I., Allende Prieto, C., \& Lambert, D. L. 2007, A\&A, 465, 271

Ramírez, I., Asplund, M., Baumann, P., Meléndez, J., \& Bensby, T. 2010, A\&A, 521, A33

Ramírez, I., Meléndez, J., Cornejo, D., Roederer, I. U., \& Fish, J. R. 2011, ApJ, 740,76

Ramírez, I., Meléndez, J., Bean, J., et al. 2014, A\&A, 572, A48

Rasio, F. A., \& Ford, E. B. 1996, Science, 274, 954

Rebull, L. M., Carlberg, J. K., Gibbs, J. C., et al. 2015, ApJ, 150, 123

Reddy, B. E., Lambert, D. L., \& Allende Prieto, C. 2006, MNRAS, 367, 1329

Reffert, S., Bergmann, C., Quirrenbach, A., Trifonov, T., \& Künstler, A. 2015, A\&A, 574, A116

Rein, H., \& Liu, S.-F. 2012, A\&A, 537, A128

Rein, H., \& Tamayo, D. 2015, MNRAS, 452, 376

Ricker, G. R. 2015, AAS/Div. Extreme Sol. Syst. Abstracts, 47, 503.01

Rowe, J. F., Bryson, S. T., Marcy, G. W., et al. 2014, ApJ, 784, 45

Rowe, J. F., Coughlin, J. L., Antoci, V., et al. 2015, ApJS, 217, 16

Saffe, C. 2011, Rev. Mex. Astron. Astrofis., 47, 3

Saffe, C., Flores, M., \& Buccino, A. 2015, A\&A, 582, A17

Saffe, C., Flores, M., Miquelarena, P., et al. 2018, A\&A, 620, A54

Saffe, C., Jofré, E., Martioli, E., et al. 2017, A\&A, 604, L4

Sato, B., Izumiura, H., Toyota, E., et al. 2008, PASJ, 60, 539

Sato, B., Omiya, M., Liu, Y., et al. 2010, PASJ, 62, 1063

Schwarz, G. 1978, Ann. Stat., 6, 461

Siess, L., \& Livio, M. 1999, MNRAS, 308, 1133

Skrutskie, M. F., Cutri, R. M., Stiening, R., et al. 2006, AJ, 131, 1163

Slumstrup, D., Grundahl, F., Brogaard, K., et al. 2017, A\&A, 604, L8

Sneden, C. A. 1973, PhD Thesis, The University of Texas at Austin, USA

Sousa, S. G., Alapini, A., Israelian, G., \& Santos, N. C. 2010, A\&A, 512, A13

Sousa, S. G., Santos, N. C., Israelian, G., et al. 2011, A\&A, 526, A99

Sousa, S. G., Santos, N. C., Adibekyan, V., Delgado-Mena, E., \& Israelian, G. 2015, A\&A, 577, A67

Sozzetti, A., Torres, G., Charbonneau, D., et al. 2007, ApJ, 664, 1190

Stassun, K. G., \& Torres, G. 2018, ApJ, 862, 61

Stassun, K. G., Collins, K. A., \& Gaudi, B. S. 2017, AJ, 153, 136

Steffen, J. H., Fabrycky, D. C., Agol, E., et al. 2013, MNRAS, 428, 1077

Takeda, Y., Sato, B., Kambe, E., et al. 2005, PASJ, 57, 109

Takeda, G., Kita, R., \& Rasio, F. A. 2008a, ApJ, 683, 1063

Takeda, Y., Sato, B., \& Murata, D. 2008b, PASJ, 60, 781

Takeda, Y., Omiya, M., Harakawa, H., \& Sato, B. 2016, PASJ, 68, 81

Thorén, P., Edvardsson, B., \& Gustafsson, B. 2004, A\&A, 425, 187

Thygesen, A. O., Frandsen, S., Bruntt, H., et al. 2012, A\&A, 543, A160

Torres, G., Andersen, J., \& Giménez, A. 2010, A\&ARv, 18, 67

Torres, G., Fischer, D. A., Sozzetti, A., et al. 2012, ApJ, 757, 161

Tucci Maia, M., Ramírez, I., Meléndez, J., et al. 2016, A\&A, 590, A32

Twicken, J. D., Jenkins, J. M., Seader, S. E., et al. 2016, AJ, 152, 158

Valenti, J. A., \& Piskunov, N. 1996, A\&AS, 118, 595

Van Eylen, V., \& Albrecht, S. 2015, ApJ, 808, 126

Van Eylen, V., Albrecht, S., Gandolfi, D., et al. 2016, AJ, 152, 143

Van Eylen, V., Albrecht, S., Huang, X., et al. 2019, AJ, 157, 61

Villaver, E., \& Livio, M. 2009, ApJ, 705, L81

Villaver, E., Livio, M., Mustill, A. J., \& Siess, L. 2014, ApJ, 794, 3

Wang, S.-G., Su, D.-Q., Chu, Y.-Q., Cui, X., \& Wang, Y.-N. 1996, Appl. Opt., 35,5155

Wright, E. L., Eisenhardt, P. R. M., Mainzer, A. K., et al. 2010, AJ, 140, 1868

Yu, J., Huber, D., Bedding, T. R., et al. 2018, ApJS, 236, 42

Zeng, L., Sasselov, D. D., \& Jacobsen, S. B. 2016, ApJ, 819, 127 


\section{Appendix A: Independent analysis on stellar mass, radius, and age}
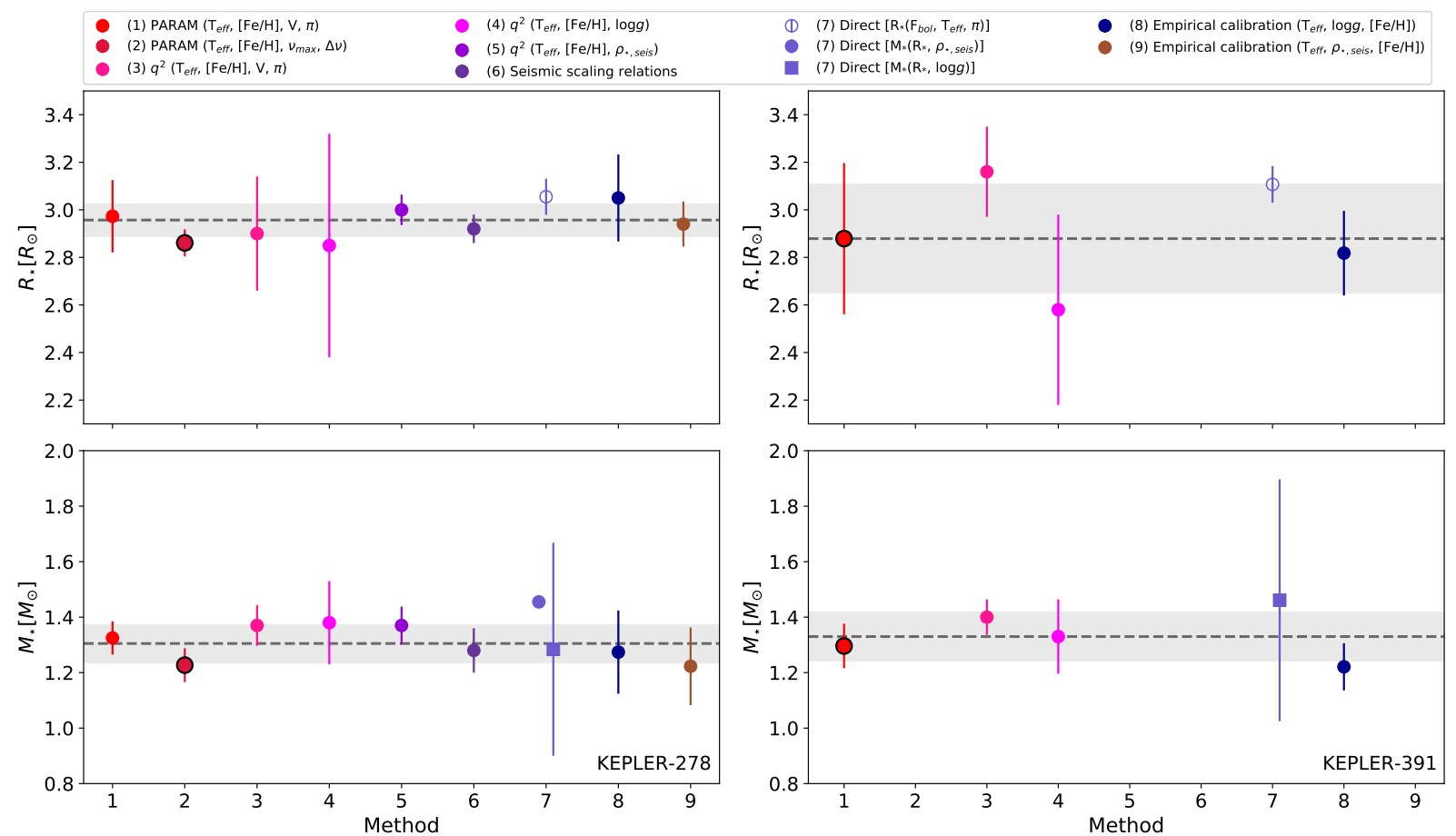

Fig. A.1. Stellar radii and masses determined by different approaches for Kepler-278 (left) and Kepler-391 (right). Dashed lines indicate the median values and the shaded areas indicate the standard deviations. The final adopted values for Kepler-278 and Kepler-391 are indicated with black edge-color circles.

In order to obtain an independent check on the stellar parameters derived from PARAM 1.3, using both DR2 Gaia parallaxes (Method 1) and asteroseismic information (Method 2), we computed masses, radii and ages of both stars with other approaches and stellar models. Figure A.1 shows the values obtained for mass and radius with all the methods, whilst Table A.1 summarizes all the estimations including ages and densities. The different methods that we applied are the following:

$q^{2}+Y Y$ models. We determined $M_{\star}, R_{\star}$, and $\tau_{\star}$ using probability distribution functions with Yonsei-Yale stellar isochrones (Demarque et al. 2004), as described in Meléndez et al. (2012) and Ramírez et al. (2014). This was acomplished via the $q^{2}$ pipeline, using as input $T_{\text {eff }},[\mathrm{Fe} / \mathrm{H}]$, parallax, and $V$ magnitude (Method 3). With this method, for Kepler-278, $q^{2}$ yields $M_{\star}=1.370 \pm 0.073 M_{\odot}, R_{\star}=2.900 \pm 0.241 R_{\odot}$, and $\tau_{\star}=4.200 \pm 0.810$ Gyr. For Kepler-391, we found $M_{\star}=1.400 \pm$ $0.064 M_{\odot}, R_{\star}=3.160 \pm 0.189 R_{\odot}$, and $\tau_{\star}=3.700 \pm 0.655 \mathrm{Gyr}$.

As another option, $q^{2}$ allows for the computation of stellar parameters from $T_{\text {eff }},[\mathrm{Fe} / \mathrm{H}]$, and surface gravity as a luminosity indicator (Method 4). Using this option, $q^{2}$ returned $M_{\star}=$ $1.380 \pm 0.149 M_{\odot}, R_{\star}=2.850 \pm 0.471 R_{\odot}$, and $\tau_{\star}=3.600 \pm 1.010$ Gyr for Kepler-278, whilst for Kepler-391 we found $M_{\star}=1.330$ $\pm 0.134 M_{\odot}, R_{\star}=2.580 \pm 0.400 R_{\odot}$, and $\tau_{\star}=4.000 \pm 1.857 \mathrm{Gyr}$.

Also, $q^{2}$ includes the possibility of using the stellar density, along with $T_{\text {eff }}$ and [Fe/H] as initial constraints (Method 5), instead of parallax or $\log g$, similar to the method described in Sozzetti et al. (2007) and Mortier et al. (2014). For Kepler278 , we used the precise asteroseismic stellar density $\left(\rho_{\star \text {,seis }}\right)$ reported by $\mathrm{H} 13$, and obtained $M_{\star}=1.370 \pm 0.086 M_{\odot}, R_{\star}=$ $3.000 \pm 0.064 R_{\odot}$, and $\tau_{\star}=4.200 \pm 1.010 \mathrm{Gyr}$, which are in good agreement with those determined using the other inputs or models.
Asteroseismic scaling relations. For Kepler-278, with available asteroseismic information, it was possible to estimate stellar mass and radius from the seismic scaling relations (Method 6) of Kallinger et al. (2010):

$$
\begin{aligned}
& \left(R_{\star} / R_{\odot}\right)=\left(v_{\max } / v_{\max , \odot}\right)\left(\Delta v / \Delta v_{\odot}\right)^{-2}\left(T_{\mathrm{eff}} / T_{\mathrm{eff}, \odot}\right)^{1 / 2} \\
& \left(M_{\star} / M_{\odot}\right)=\left(v_{\max } / v_{\max , \odot}\right)^{3}\left(\Delta v / \Delta v_{\odot}\right)^{-4}\left(T_{\mathrm{eff}} / T_{\mathrm{eff}, \odot}\right)^{3 / 2} .
\end{aligned}
$$

As before, here we employed our spectroscopic $T_{\text {eff }}$ and asteroseismic parameters of H13. In excellent agreement with the results obtained with PARAM, these equations returned: $M_{\star}=$ $1.283 \pm 0.077 M_{\odot}, R_{\star}=2.922 \pm 0.058 R_{\odot}$.

Direct mass and radius. As another test, we derived stellar radii and masses using direct observables (Method 7), following the procedure described in Stassun et al. (2017). Briefly, the stellar angular radius $\Theta$ is computed from direct observables such as the bolometric flux $F_{\text {bol }}$, the effective temperature, and the parallax $\pi$ according to,

$\Theta=F_{\text {bol }}^{1 / 2} \times\left(\frac{2341}{T_{\text {eff }}}\right)^{2}$,

where $F_{\text {bol }}$ is in $10^{-8} \mathrm{erg} \mathrm{cm}^{-2} \mathrm{~s}^{-1}, T_{\text {eff }}$ is in $\mathrm{K}$, and $\Theta$ in mas. Then, the linear radius can be obtained via,

$R_{\star}=107.47 \frac{\Theta}{\pi}$,

where $\pi$ is in mas and $R_{\star}$ in solar radii. We measured $F_{\text {bol }}$ in our Kepler stars by fitting atmosphere models to the observed SEDs using the VOSA interface as is detailed in Sect. 3.1.1. Using 
Table A.1. Stellar physical parameters obtained by the different techniques for Kepler-278 and Kepler-391.

\begin{tabular}{|c|c|c|c|c|c|c|}
\hline \# & Method & Input parameters & $M_{\star}\left[M_{\odot}\right]$ & $R_{\star}\left[R_{\odot}\right]$ & $\tau_{\star}[\mathrm{Gyr}]$ & $\rho_{\star}\left[\mathrm{g} \mathrm{cm}^{-3}\right]$ \\
\hline \multicolumn{7}{|c|}{ Kepler-278 } \\
\hline 1 & PARAM 1.3 (Parsec iscochrones) & $T_{\text {eff }},[\mathrm{Fe} / \mathrm{H}], V, \pi$ & $1.325 \pm 0.060$ & $2.973 \pm 0.152$ & $4.466 \pm 0.630$ & $0.071 \pm 0.006$ \\
\hline 2 & PARAM 1.3 (Parsec iscochrones) & $T_{\mathrm{eff}},[\mathrm{Fe} / \mathrm{H}], \Delta v, v_{\max }$ & $1.227 \pm 0.061$ & $2.861 \pm 0.057$ & $5.761 \pm 1.019$ & $0.074 \pm 0.005$ \\
\hline 3 & $q^{2}$ (Yonsei-Yale isochrones) & $T_{\mathrm{eff}},[\mathrm{Fe} / \mathrm{H}], \log g$ & $1.370 \pm 0.073$ & $2.900 \pm 0.241$ & $4.200 \pm 0.810$ & $0.079 \pm 0.009$ \\
\hline 4 & $q^{2}$ (Yonsei-Yale isochrones) & $T_{\mathrm{eff}},[\mathrm{Fe} / \mathrm{H}], V, \pi$ & $1.380 \pm 0.150$ & $2.850 \pm 0.471$ & $3.600 \pm 1.500$ & $0.084 \pm 0.019$ \\
\hline 6 & Asteroseismic scaling relations ${ }^{(a)}$ & $T_{\text {eff }}, \Delta v, v_{\max }$ & $1.283 \pm 0.077$ & $2.922 \pm 0.058$ & N/A & $0.072 \pm 0.005$ \\
\hline 7 & Direct $^{(b)}$ & $R_{\star}\left(F_{\mathrm{bol}}, T_{\mathrm{eff}}, \pi\right) ; M_{\star}\left(R_{\star}, \rho_{\star, \mathrm{seis}}\right)$ & $1.455 \pm 0.019$ & $3.055 \pm 0.076$ & N/A & $0.072 \pm 0.002$ \\
\hline 7 & Direct $^{(b)}$ & $R_{\star}\left(F_{\text {bol }}, T_{\text {eff }}, \pi\right) ; M_{\star}\left(R_{\star}, \log g\right)$ & $1.284 \pm 0.384$ & $3.055 \pm 0.076$ & N/A & $0.063 \pm 0.020$ \\
\hline 8 & Empirical calibration $^{(c)}$ & $T_{\mathrm{eff}},[\mathrm{Fe} / \mathrm{H}], \log g$ & $1.274 \pm 0.150$ & $3.005 \pm 0.183$ & N/A & $0.066 \pm 0.011$ \\
\hline 9 & Empirical calibration ${ }^{(d)}$ & $T_{\mathrm{eff}},[\mathrm{Fe} / \mathrm{H}], \rho_{\star, \text { seis }}$ & $1.223 \pm 0.143$ & $2.940 \pm 0.095$ & N/A & $0.068 \pm 0.009$ \\
\hline \multicolumn{7}{|c|}{ Kepler-391 } \\
\hline 1 & PARAM 1.3 (Parsec iscochrones) & $T_{\text {eff }},[\mathrm{Fe} / \mathrm{H}], V, \pi$ & $1.296 \pm 0.080$ & $2.879 \pm 0.318$ & $4.365 \pm 0.899$ & $0.077 \pm 0.011$ \\
\hline 2 & PARAM 1.3 (Parsec iscochrones) & $T_{\mathrm{eff}},[\mathrm{Fe} / \mathrm{H}], \Delta v, v_{\max }$ & N/A & N/A & N/A & N/A \\
\hline 3 & $q^{2}$ (Yonsei-Yale isochrones) & $T_{\mathrm{eff}},[\mathrm{Fe} / \mathrm{H}], \log g$ & $1.400 \pm 0.064$ & $3.160 \pm 0.189$ & $3.700 \pm 0.655$ & $0.063 \pm 0.006$ \\
\hline 4 & $q^{2}$ (Yonsei-Yale isochrones) & $T_{\mathrm{eff}},[\mathrm{Fe} / \mathrm{H}], V, \pi$ & $1.330 \pm 0.134$ & $2.580 \pm 0.400$ & $4.000 \pm 1.857$ & $0.109 \pm 0.023$ \\
\hline 5 & $q^{2}$ (Yonsei-Yale isochrones) & $T_{\mathrm{eff}},[\mathrm{Fe} / \mathrm{H}], \rho_{\star, \text { seis }}$ & N/A & N/A & N/A & N/A \\
\hline 6 & Asteroseismic scaling relations ${ }^{(a)}$ & $T_{\mathrm{eff}}, \Delta v, v_{\max }$ & N/A & N/A & N/A & N/A \\
\hline 7 & Direct $^{(b)}$ & $R_{\star}\left(F_{\mathrm{bol}}, T_{\mathrm{eff}}, \pi\right) ; M_{\star}\left(R_{\star}, \rho_{\star, \mathrm{seis}}\right)$ & N/A & N/A & N/A & N/A \\
\hline 7 & Direct $^{(b)}$ & $R_{\star}\left(F_{\mathrm{bol}}, T_{\mathrm{eff}}, \pi\right) ; M_{\star}\left(R_{\star}, \log g\right)$ & $1.461 \pm 0.436$ & $3.107 \pm 0.077$ & N/A & $0.069 \pm 0.022$ \\
\hline 8 & Empirical calibration $^{(c)}$ & $T_{\mathrm{eff}},[\mathrm{Fe} / \mathrm{H}], \log g$ & $1.221 \pm 0.085$ & $2.818 \pm 0.178$ & N/A & $0.077 \pm 0.009$ \\
\hline 9 & Empirical calibration $^{(d)}$ & $T_{\mathrm{eff}},[\mathrm{Fe} / \mathrm{H}], \rho_{\star, \text { seis }}$ & N/A & N/A & N/A & $0.000 \pm 0.000$ \\
\hline
\end{tabular}

Notes. ${ }^{(a)}$ Asteroseismic scaling relations from Kallinger et al. (2010). ${ }^{(b)}$ Method detailed in Stassun et al. (2017). ${ }^{(c)}$ Empirical relations of Torres et al. (2010). ${ }^{(d)}$ Empirical relations of Enoch et al. (2010).

the derived $F_{\text {bol }}$, spectroscopic $T_{\text {eff }}$ and DR2 Gaia parallaxes in Eqs. (A.4) and (A.5), we obtained $R_{\star}=3.055 \pm 0.076 R_{\odot}$ and $R_{\star}=3.107 \pm 0.077 R_{\odot}$ for Kepler-278 and Kepler-391, respectively. In both cases the model independent radii are in good agreement with those derived from Yonsei-Yale and PARSEC stellar models.

A direct measure of the stellar mass can be obtained by combining the empirically computed stellar radius with the stellar density obtained from asteroseismology or from the transit light curve analysis. For Kepler-278, we derived $M_{\star}=1.455 \pm$ $0.019 M_{\odot}$ from the seismic density reported by $\mathrm{H} 13$ which agrees with those computed from stellar models. Given that both Kepler-278 and Kepler-391 present low S/N Kepler light curves (see Sect. 4), we do not obtain stellar densities from these data.

As suggested by Stassun et al. (2017), another possibility to derive a model-independent stellar mass is by using the direct $R_{\star}$ in combination with the spectroscopic $\log g$ via the formula:

$\frac{M_{\star}}{M_{\odot}}=\left(\frac{R_{\star}}{R_{\odot}}\right)^{2} 10^{\log g-\log g_{\odot}}$,

where $\log g_{\odot}=4.44$ dex. For Kepler-278 we derived $M_{\star}=$ $1.284 \pm 0.384 M_{\odot}$ and $M_{\star}=1.461 \pm 0.436 M_{\odot}$ for Kepler-391.

Empirical calibration 1. We computed $M_{\star}$ and $R_{\star}$ from the empirical relations of Torres et al. (2010) that link $T_{\text {eff }}, \log g$, and $[\mathrm{Fe} / \mathrm{H}]$ to the stellar mass and radius (Method 8). The relations were calibrated based on the precisely measured masses and radii (3\% or better) of 95 eclipsing binaries. The results based on this calibration are $M_{\star}=1.274 \pm 0.150 M_{\odot}$ and $R_{\star}=3.005 \pm$ $0.183 R_{\odot}$ for Kepler-278, and of $M_{\star}=1.221 \pm 0.085 M_{\odot}$ and $R_{\star}=2.818 \pm 0.178 R_{\odot}$ for Kepler-391. For both stars, the results are in excellent agreement with those obtained with PARAM and the other methods.
Empirical calibration 2. Finally, we tested our results via the empirical calibration of Enoch et al. (2010) which is similar to that of Torres et al. (2010), but uses $\rho_{\star}$ instead of $\log g$ as input (Method 9). Also, their equations were calibrated with the same 95 eclipsing binaries from Torres et al. (2010). For Kepler-278, we obtain $M_{\star}=1.223 \pm 0.143 M_{\odot}$ and $R_{\star}=2.940 \pm$ $0.095 R_{\odot}$ from the $\rho_{\star \text {, seis }}$ value by $\mathrm{H} 13$ as input. These results are in good agreement with those obtained from PARAM. All values for the stellar radii, masses, ages, and stellar densities that result from the different techniques and models are summarized in Table A.1. As can be seen from this table and in Fig A.1, there is generally good agreement between the masses and radii obtained from PARAM + PARSEC isochrones and those derived from most of the other techniques and stellar models.

\section{Ages from the $[\mathrm{Y} / \mathrm{Mg}]$ ratio and lithium abundance}

It has been proven that the stellar abundances of Y and $\mathrm{Mg}$ can be used to estimate stellar ages of solar-type stars through the [Y/Mg] ratio (Nissen 2015, 2016; Tucci Maia et al. 2016; Feltzing et al. 2017). Moreover, recently Slumstrup et al. (2017) found that the $[\mathrm{Y} / \mathrm{Mg}]$ ratio is also a good indicator of age for evolved stars. Therefore, considering the age- $[\mathrm{Y} / \mathrm{Mg}]$ relation from Tucci Maia et al. (2016), we obtain an age of $5.95 \pm 2.57$ Gyr for Kepler278 from $[\mathrm{Y} / \mathrm{Mg}]=-0.06$, whilst for Kepler-391, with $[\mathrm{Y} / \mathrm{Mg}]=$ 0.02 , we derive an age of $4.14 \pm 2.58$ Gyr.

We also derived the stellar ages employing the lithium-age relation from Carlos et al. (2016). We estimated that Kepler-278, with a sub-solar lithium abundance, has an age of $5.5 \pm 1.3 \mathrm{Gyr}$, whilst the Kepler-391's higher lithium abundance implies a subsolar age of $3.64 \pm 1.2$ Gyr. As can be seen from Sect. 3.3 and in the previous section of this Appendix, these independent stellar ages are in good agreement with the values derived from the isochrones analysis. 


\section{Appendix B: Additional figures and tables}
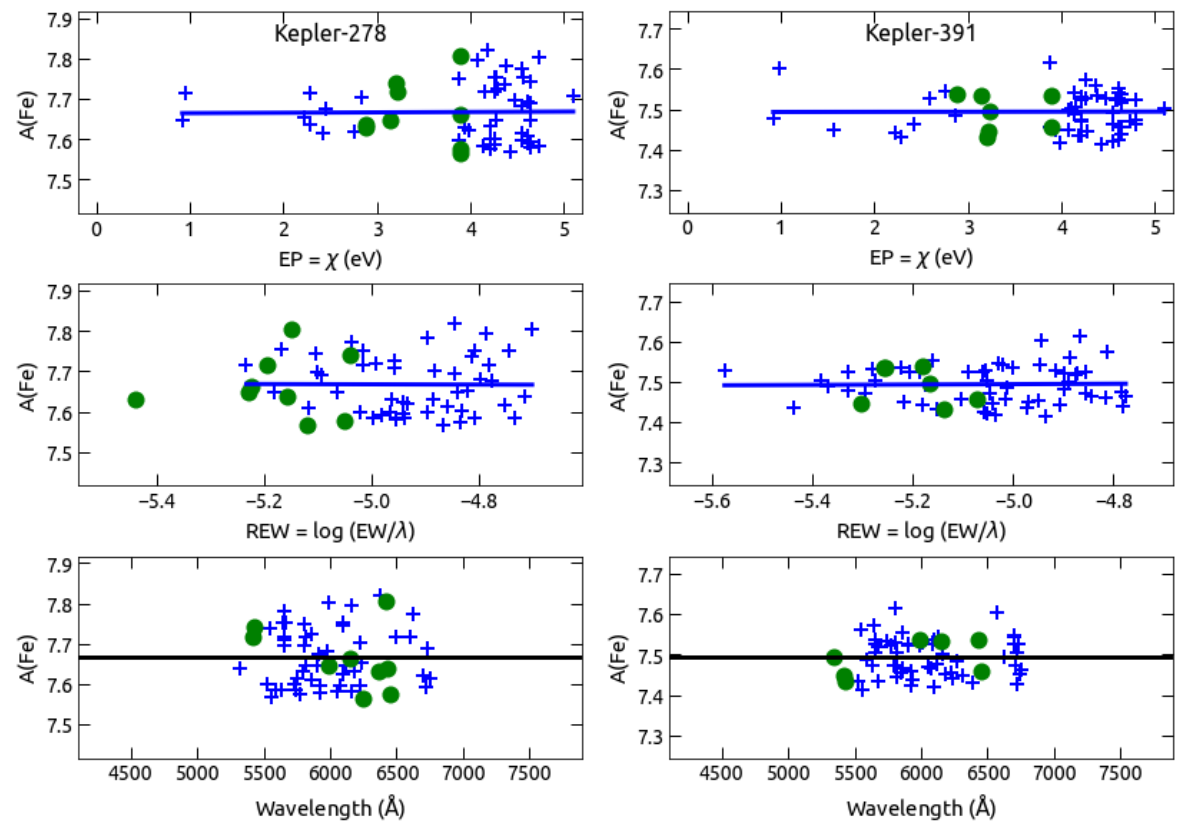

Fig. B.1. Iron abundance as a function of the spectral lines' excitation potential (upper panels), reduced EWs (middle panels), and wavelength (bottom panels) for Kepler-278 (left) and Kepler-391 (right). Blue crosses (green circles) correspond to Fe I (Fe II). Top and middle panels: solid lines are linear fits to the $\mathrm{Fe} I$ data. Lower panel: the solid lines indicate the average $\mathrm{A}(\mathrm{Fe})$ values.

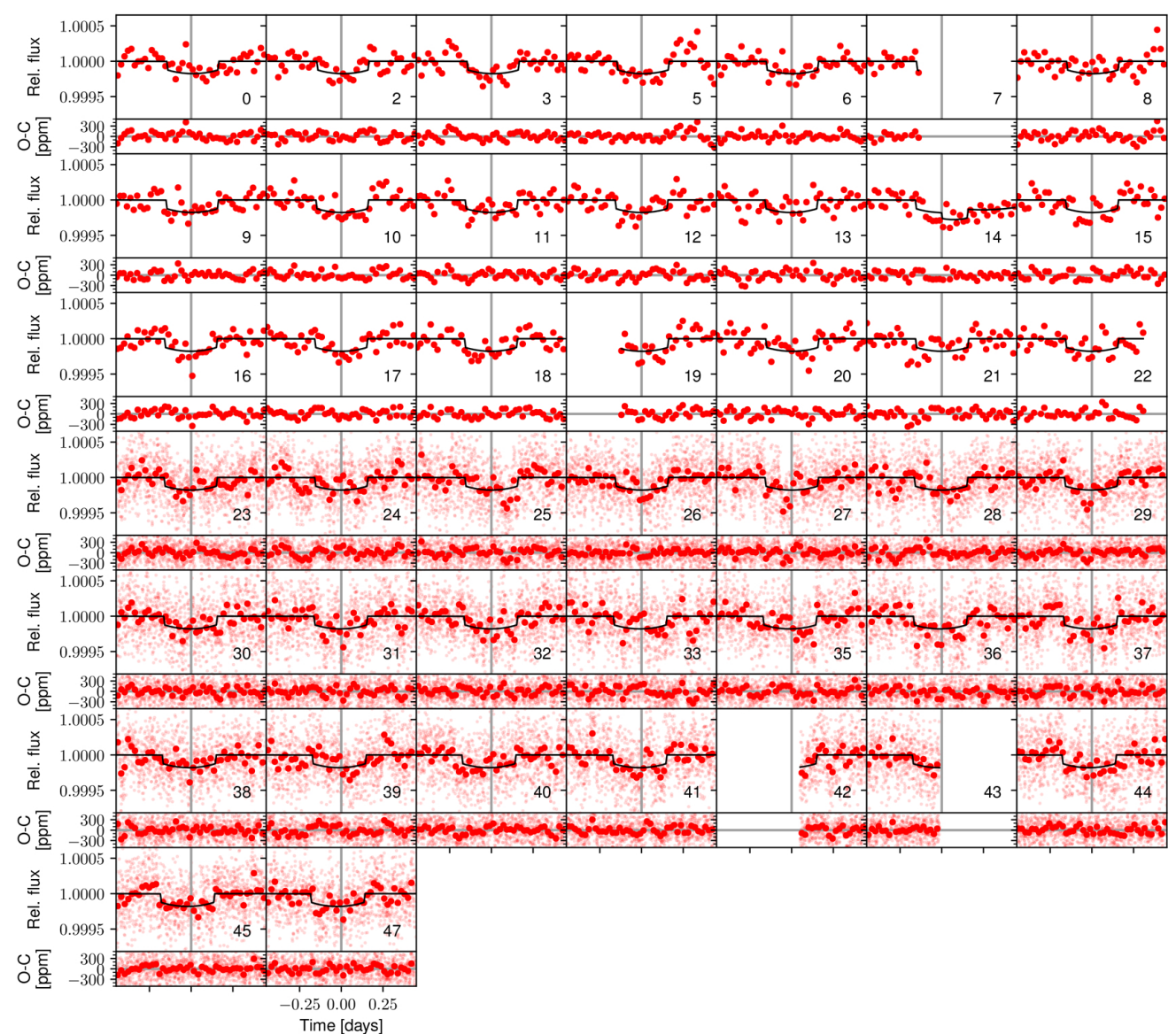

Fig. B.2. Transits of Kepler-278b observed by Kepler. Dots represent the individual short-cadence observations and larger circles are 30-min averaged values. In those panels without short-cadence points, the circles represent the long-cadence data. Each panel is labelled with the transit epoch, and centered relative to a linear ephemeris (indicated by the vertical grey lines). The model distribution is constructed from 1000 random MCMC steps. The black line denotes the median model. In the lower part of each panel the residuals after subtracting the model to the observed data are shown. 


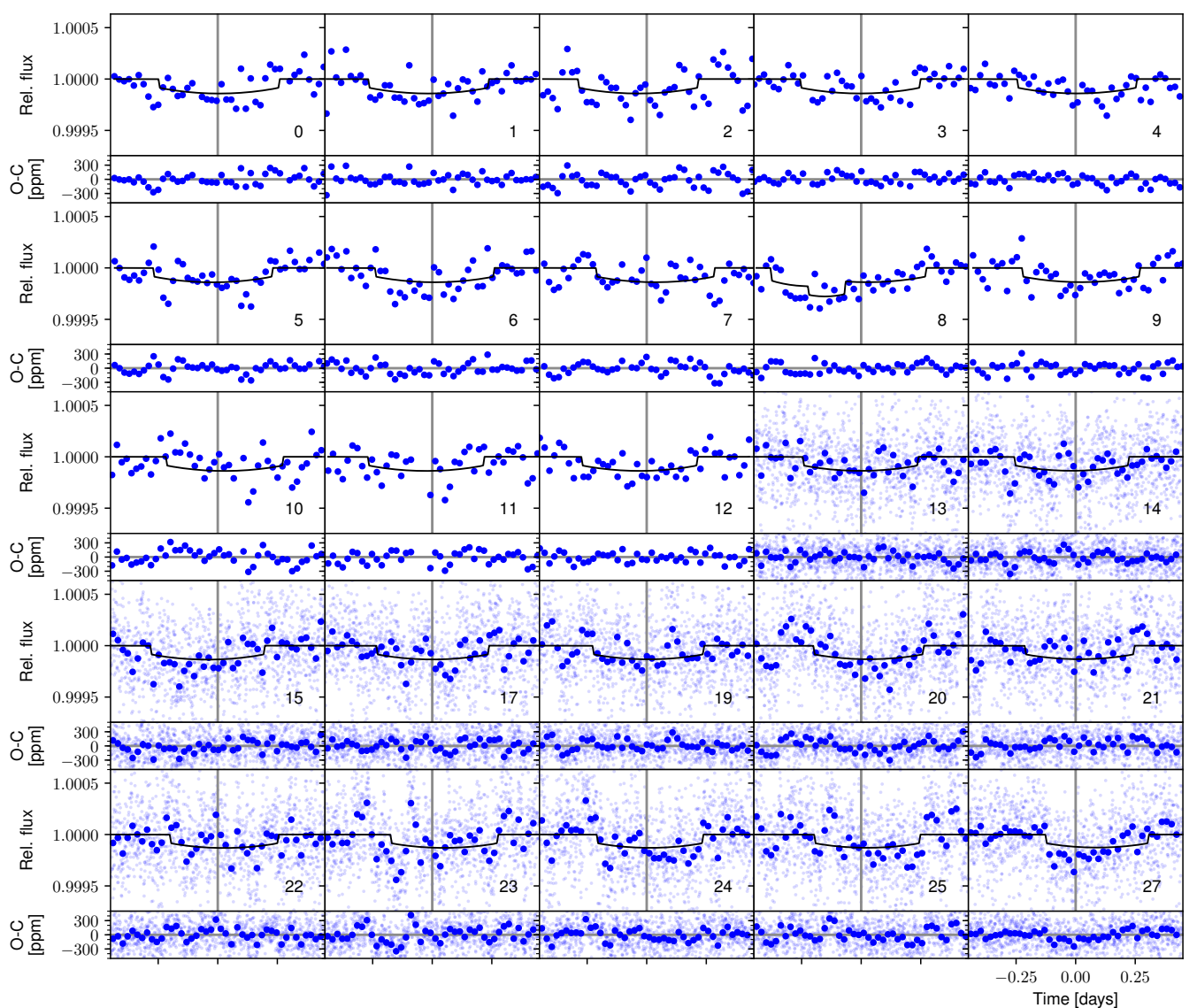

Fig. B.3. Same as Fig. B.2 for Kepler-278c.

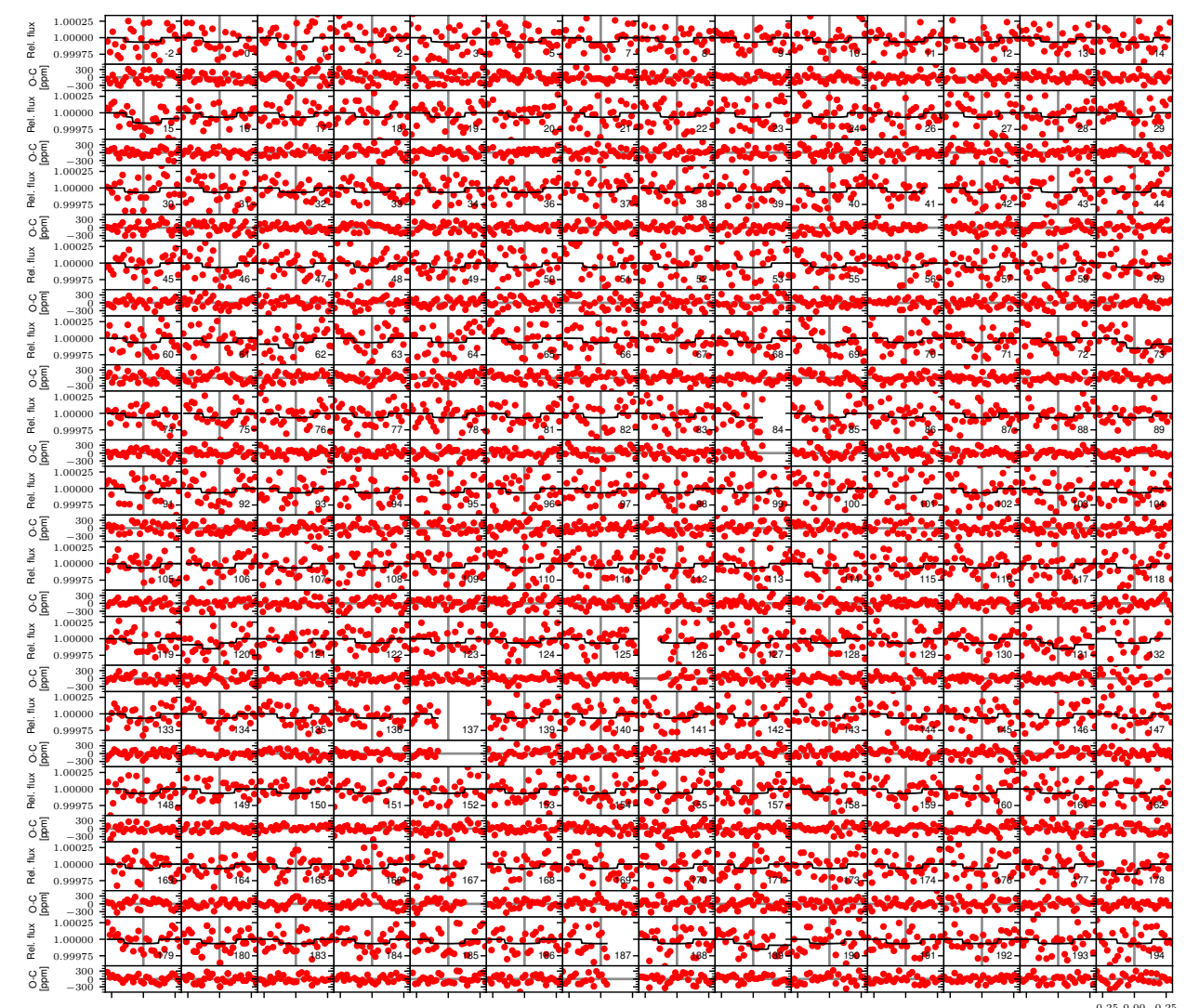

Fig. B.4. Same as Fig. B.2 for Kepler-391b. 


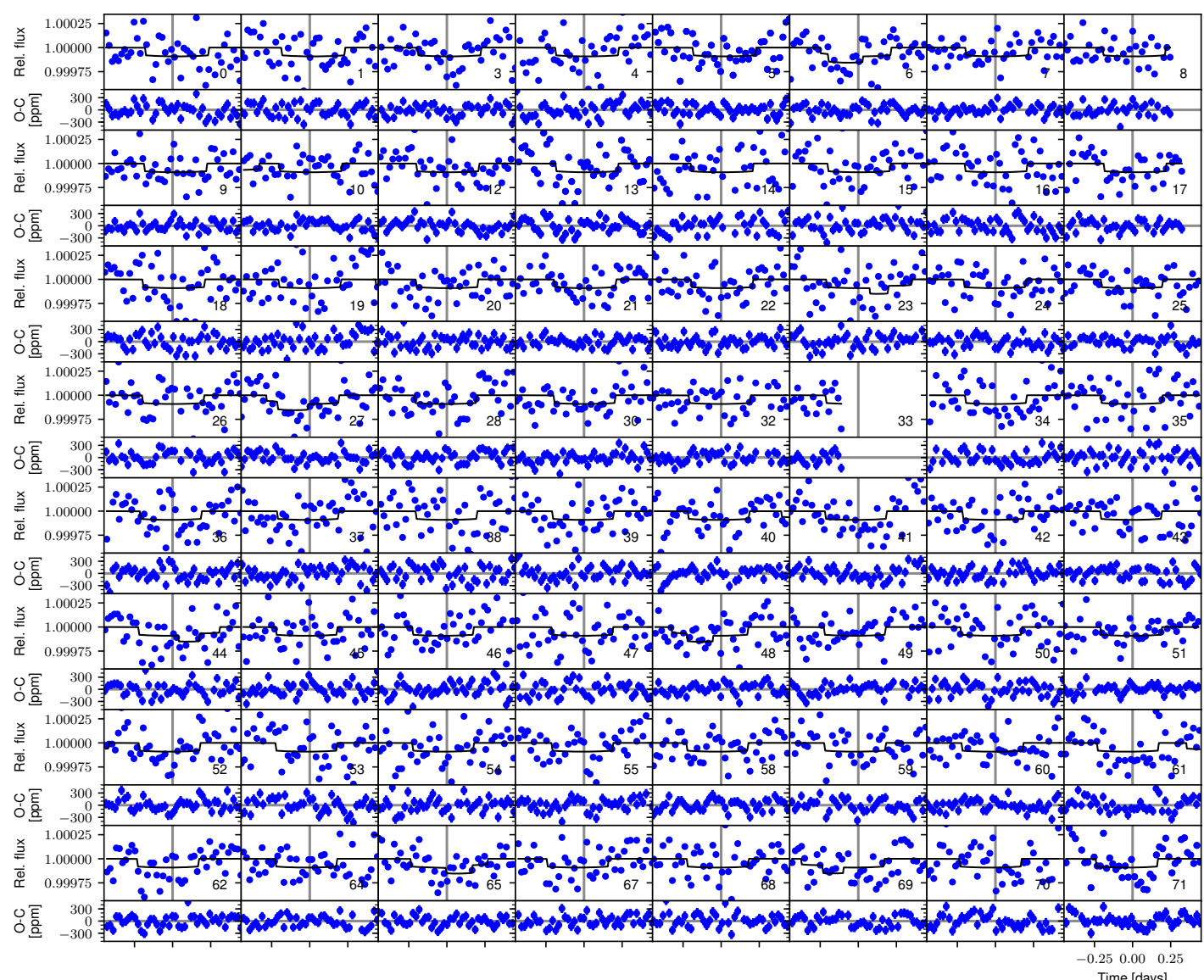

Fig. B.5. Same as Fig. B.2 for Kepler-391c. 


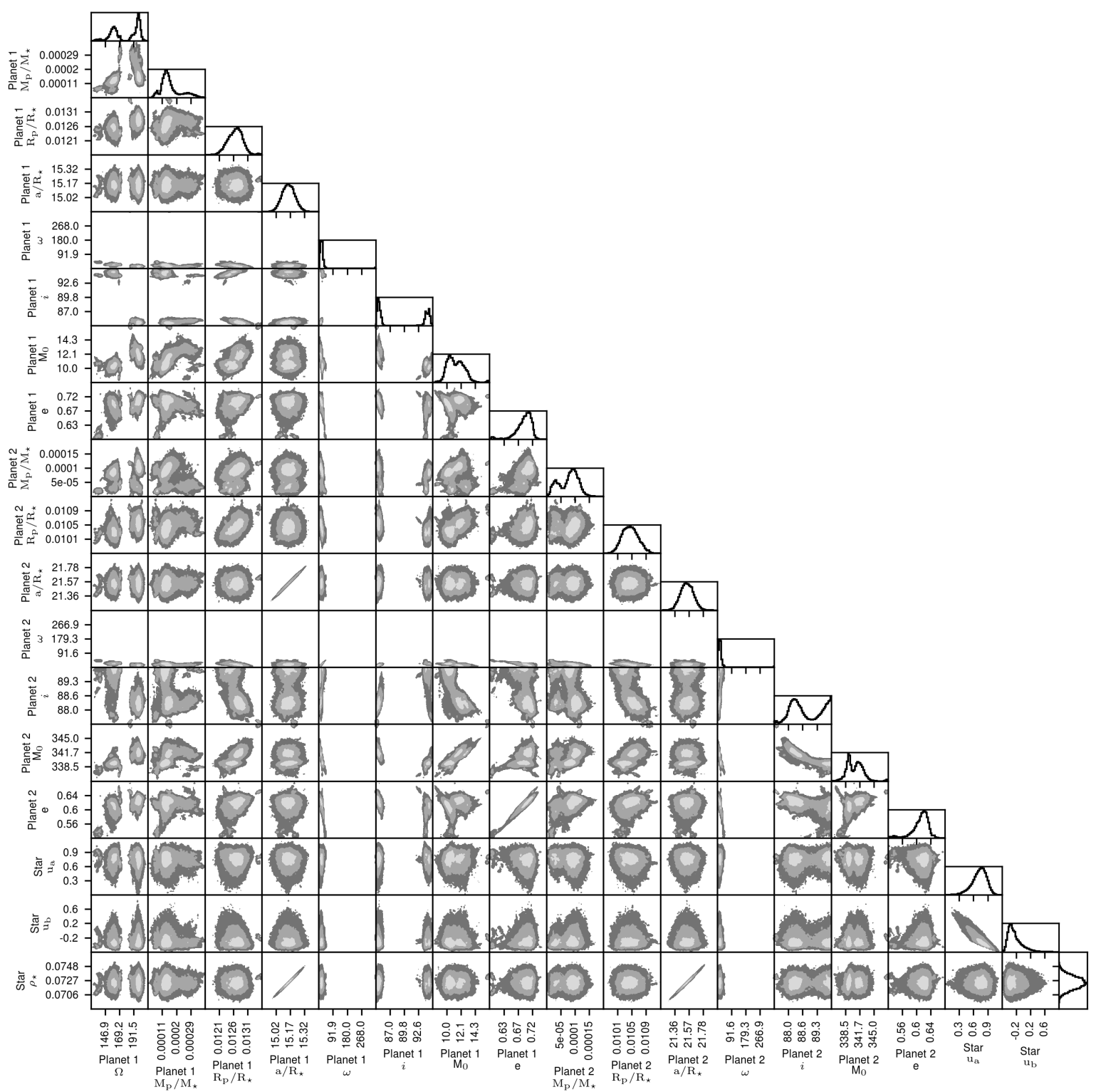

Fig. B.6. 2D projections of the joint posterior samples obtained with the MCMC algorithm for planets around Kepler-278. The 39.3, 86.5, and 98.9 per cent 2D joint confidence regions (in the case of a Gaussian posterior, these regions project onto the one-dimensional (1D) 1, 2, and $3 \sigma$ intervals) are denoted by three different grey levels. The 1D histogram of each parameter is shown at the top of each column, except for the parameter on the last line that is shown at the end of the line. Units are the same as in Table 2. 
E. Jofré et al.: Stellar and planetary characterization of the multiplanet systems Kepler-278 and Kepler-391

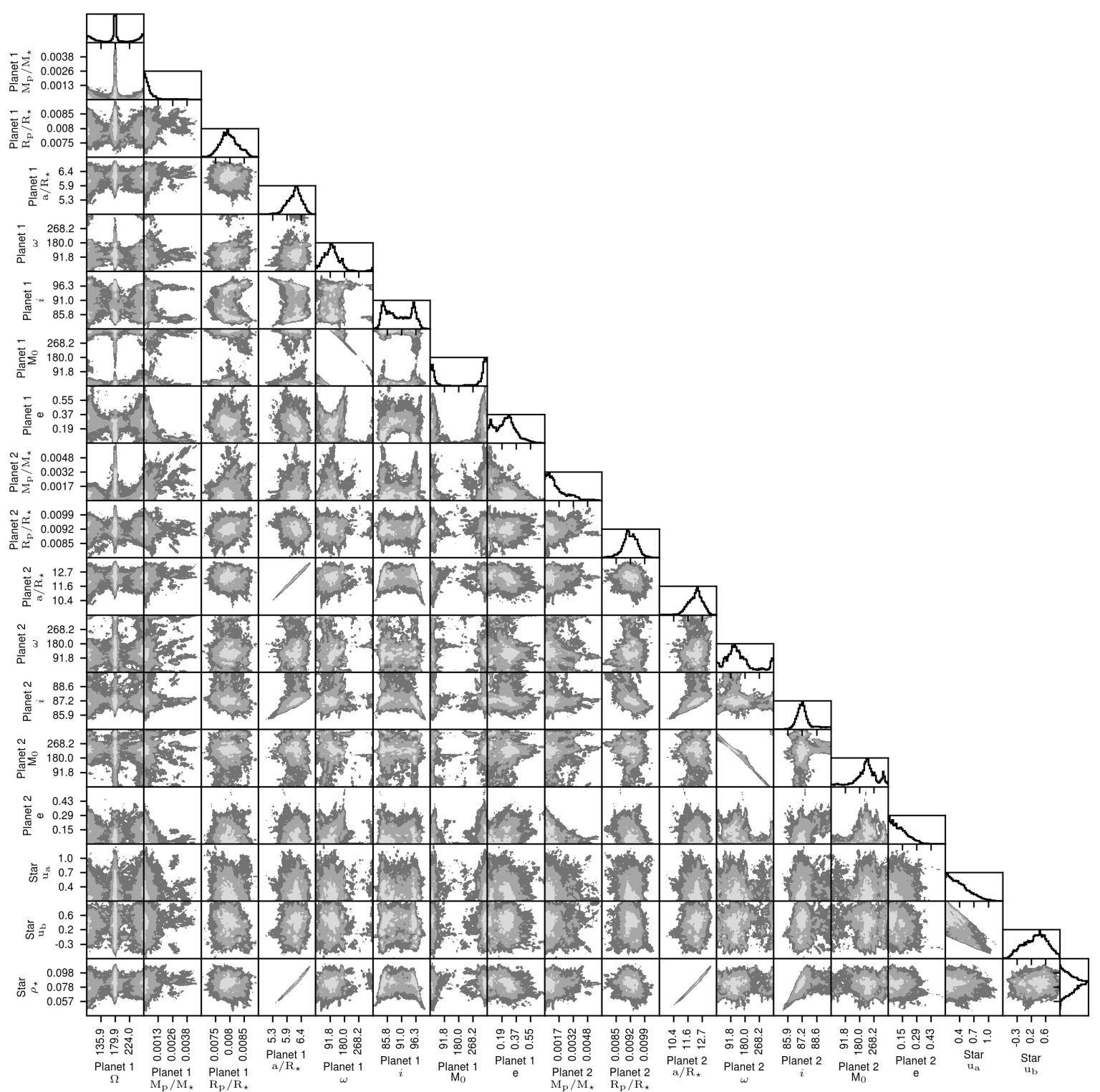

Fig. B.7. Same as Fig. B.6 but for planets around Kepler-391. 
Table B.1. Elemental abundances of Kepler-278 and Kepler-391.

\begin{tabular}{|c|c|c|c|c|c|c|c|c|c|c|c|c|c|}
\hline \multicolumn{7}{|c|}{ Kepler-278 } & \multicolumn{7}{|c|}{ Kepler-391 } \\
\hline Species & $\mathrm{A}(\mathrm{X})$ & {$[\mathrm{X} / \mathrm{H}]$} & {$[\mathrm{X} / \mathrm{Fe}]$} & $\sigma_{\text {line }}$ & $\sigma_{\text {pars }}$ & $\sigma_{\text {tot }}$ & Species & $\mathrm{A}(\mathrm{X})$ & {$[\mathrm{X} / \mathrm{H}]$} & {$[\mathrm{X} / \mathrm{Fe}]$} & $\sigma_{\text {line }}$ & $\sigma_{\text {pars }}$ & $\sigma_{\text {tot }}$ \\
\hline $\operatorname{Li}_{\text {NLTE }}$ & 0.87 & -0.18 & -0.40 & & 0.1 & 0.10 & $\mathrm{Li}_{\mathrm{NLTE}}$ & 1.29 & 0.24 & 0.20 & & 0.09 & 0.09 \\
\hline C & 8.54 & 0.11 & -0.11 & 0.03 & 0.09 & 0.10 & $\mathrm{C}$ & 8.31 & -0.12 & -0.16 & 0.10 & 0.07 & 0.12 \\
\hline $\mathrm{N}$ & 8.17 & 0.34 & 0.12 & 0.09 & 0.13 & 0.16 & $\mathrm{~N}$ & 7.84 & 0.01 & -0.03 & 0.10 & 0.13 & 0.16 \\
\hline $\mathrm{O}_{\text {NLTE }}$ & 8.84 & 0.15 & -0.07 & 0.04 & 0.11 & 0.12 & $\mathrm{O}_{\mathrm{NLTE}}$ & 8.71 & 0.02 & -0.02 & 0.02 & 0.10 & 0.11 \\
\hline${ }^{12} \mathrm{C} /{ }^{13} \mathrm{C}$ & $>40$ & & & & & & ${ }^{12} \mathrm{C} /{ }^{13} \mathrm{C}$ & $>40$ & & & & & \\
\hline $\mathrm{Na}_{\mathrm{NLTE}}$ & 6.63 & 0.39 & 0.17 & 0.01 & 0.05 & 0.05 & $\mathrm{Na}_{\text {NLTE }}$ & 6.33 & 0.09 & 0.05 & 0.01 & 0.05 & 0.06 \\
\hline $\mathrm{Mg}$ & 7.84 & 0.24 & 0.02 & 0.02 & 0.02 & 0.03 & $\mathrm{Mg}$ & 7.64 & 0.04 & 0.00 & 0.05 & 0.03 & 0.06 \\
\hline $\mathrm{Al}$ & 6.83 & 0.38 & 0.16 & 0.01 & 0.04 & 0.04 & $\mathrm{Al}$ & 6.55 & 0.10 & 0.06 & 0.09 & 0.05 & 0.10 \\
\hline $\mathrm{Si}$ & 7.75 & 0.24 & 0.02 & 0.02 & 0.03 & 0.04 & $\mathrm{Si}$ & 7.58 & 0.07 & 0.03 & 0.02 & 0.02 & 0.03 \\
\hline $\mathrm{S}$ & 7.21 & 0.09 & -0.13 & & 0.10 & 0.10 & $\mathrm{~S}$ & 7.02 & -0.10 & -0.14 & & 0.09 & 0.09 \\
\hline $\mathrm{Ca}$ & 6.56 & 0.22 & 0.00 & 0.02 & 0.07 & 0.07 & $\mathrm{Ca}$ & 6.40 & 0.06 & 0.02 & 0.03 & 0.09 & 0.09 \\
\hline Sc II & 3.29 & 0.14 & -0.08 & 0.06 & 0.06 & 0.08 & Sc II & 3.12 & -0.03 & -0.07 & 0.02 & 0.04 & 0.05 \\
\hline $\mathrm{Ti}$ & 5.24 & 0.29 & 0.07 & 0.02 & 0.09 & 0.09 & $\mathrm{Ti}$ & 5.07 & 0.12 & 0.08 & 0.02 & 0.10 & 0.10 \\
\hline V & 4.24 & 0.31 & 0.09 & 0.02 & 0.09 & 0.09 & V & 4.00 & 0.07 & 0.03 & 0.05 & 0.11 & 0.12 \\
\hline $\mathrm{Cr}$ & 5.87 & 0.23 & 0.01 & 0.02 & 0.06 & 0.06 & $\mathrm{Cr}$ & 5.73 & 0.09 & 0.05 & 0.04 & 0.07 & 0.08 \\
\hline $\mathrm{Mn}$ & 5.73 & 0.30 & 0.08 & 0.09 & 0.06 & 0.11 & $\mathrm{Mn}$ & 5.50 & 0.07 & 0.03 & 0.05 & 0.07 & 0.08 \\
\hline $\mathrm{Fe}$ & 7.67 & 0.22 & 0.00 & 0.01 & 0.04 & 0.04 & $\mathrm{Fe}$ & 7.49 & 0.04 & 0.00 & 0.01 & 0.03 & 0.04 \\
\hline Co & 5.25 & 0.26 & 0.04 & 0.03 & 0.04 & 0.04 & Co & 4.99 & 0.00 & -0.04 & 0.03 & 0.04 & 0.05 \\
\hline $\mathrm{Ni}$ & 6.44 & 0.22 & 0.00 & 0.02 & 0.04 & 0.04 & $\mathrm{Ni}$ & 6.25 & 0.03 & -0.01 & 0.01 & 0.04 & 0.04 \\
\hline $\mathrm{Cu}$ & 4.60 & 0.41 & 0.19 & 0.15 & 0.04 & 0.15 & $\mathrm{Cu}$ & 4.40 & 0.21 & 0.17 & 0.11 & 0.03 & 0.12 \\
\hline $\mathrm{Zn}$ & 4.74 & 0.18 & -0.04 & 0.03 & 0.05 & 0.06 & $\mathrm{Zn}$ & 4.53 & -0.03 & -0.07 & 0.03 & 0.04 & 0.05 \\
\hline $\mathrm{Sr}$ & 2.94 & 0.07 & -0.15 & 0.15 & 0.12 & 0.19 & $\mathrm{Sr}$ & 2.77 & -0.10 & -0.14 & 0.11 & 0.12 & 0.16 \\
\hline Y II & 2.39 & 0.26 & 0.04 & 0.04 & 0.06 & 0.08 & Y II & 2.27 & 0.06 & 0.02 & 0.03 & 0.03 & 0.05 \\
\hline $\mathrm{Zr}$ & 2.89 & 0.31 & 0.09 & 0.03 & 0.06 & 0.07 & $\mathrm{Zr}$ & 2.69 & 0.11 & 0.07 & 0.10 & 0.04 & 0.11 \\
\hline Ba II & 2.37 & 0.19 & -0.03 & 0.03 & 0.06 & 0.07 & Ba II & 2.14 & -0.04 & -0.08 & 0.03 & 0.04 & 0.05 \\
\hline Ce II & 1.87 & 0.29 & 0.07 & 0.07 & 0.06 & 0.10 & Ce II & 1.79 & 0.21 & 0.17 & 0.02 & 0.03 & 0.04 \\
\hline
\end{tabular}

